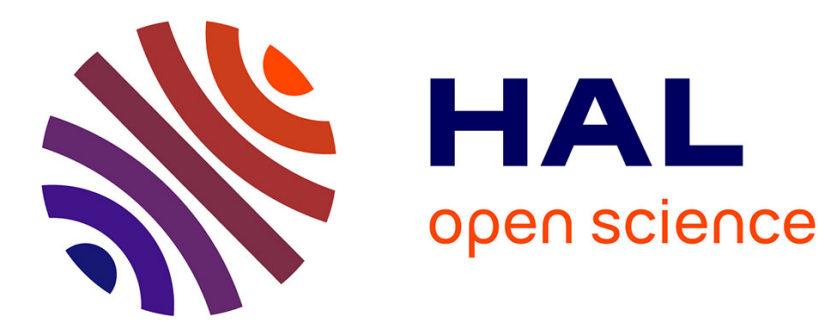

\title{
Heat coefficient $a \_4$ for non minimal Laplace type operators
}

\author{
Bruno Iochum, Thierry Masson
}

\section{To cite this version:}

Bruno Iochum, Thierry Masson. Heat coefficient $a \_4$ for non minimal Laplace type operators. Journal of Geometry and Physics, 2019, 141, pp.120-146. 10.1016/j.geomphys.2019.03.002 . hal-01974457

\section{HAL Id: hal-01974457 \\ https://hal.science/hal-01974457}

Submitted on 22 Oct 2021

HAL is a multi-disciplinary open access archive for the deposit and dissemination of scientific research documents, whether they are published or not. The documents may come from teaching and research institutions in France or abroad, or from public or private research centers.
L'archive ouverte pluridisciplinaire HAL, est destinée au dépôt et à la diffusion de documents scientifiques de niveau recherche, publiés ou non, émanant des établissements d'enseignement et de recherche français ou étrangers, des laboratoires publics ou privés.

\section{다)(1) $(5$}

Distributed under a Creative Commons Attribution - NonCommercial| 4.0 International 


\title{
Heat coefficient $a_{4}$ for non minimal Laplace type operators
}

\author{
B. Iochum, T. Masson \\ Centre de Physique Théorique ${ }^{1}$ \\ Aix Marseille Univ, Université de Toulon, CNRS, CPT, Marseille, France
}

\begin{abstract}
Given a smooth hermitean vector bundle $V$ of fiber $\mathbb{C}^{N}$ over a compact Riemannian manifold and $\nabla$ a covariant derivative on $V$, let $P=-\left(|g|^{-1 / 2} \nabla_{\mu}|g|^{1 / 2} g^{\mu v} u \nabla_{\nu}+p^{\mu} \nabla_{\mu}+q\right)$ be a non minimal Laplace type operator acting on smooth sections of $V$ where $u, p^{v}, q$ are $M_{N}(\mathbb{C})$-valued functions with $u$ positive and invertible. For any $a \in \Gamma(\operatorname{End}(V))$, we consider the asymptotics $\operatorname{Tr} a e^{-t P} \sim_{t \downarrow 0} \sum_{r=0}^{\infty} a_{r}(a, P) t^{(r-d) / 2}$ where the coefficients $a_{r}(a, P)$ can be written as an integral of the functions $a_{r}(a, P)(x)=\operatorname{tr}\left[a(x) \mathscr{R}_{r}(x)\right]$.

This paper revisits the previous computation of $\mathscr{R}_{2}$ by the authors and is mainly devoted to a computation of $\mathscr{R}_{4}$. The results are presented with $u$-dependent operators which are universal (i.e. $P$-independent) and which act on tensor products of $u, p^{\mu}, q$ and their derivatives via (also universal) spectral functions which are fully described.
\end{abstract}

\section{An introduction to the method}

Let $V$ be a smooth hermitean vector bundle $V$ of fiber $\mathbb{C}^{N}$ over a compact $d$-dimensional boundaryless Riemannian manifold $(M, g)$ and let $P$ be a non minimal Laplace type operator acting on the smooth sections $\Gamma(V)$, written locally as the partial differential operator $P=-\left[g^{\mu v} u(x) \partial_{\mu} \partial_{v}+v^{\mu}(x) \partial_{\mu}+w(x)\right]$ where $x \in M$ and $u, v^{\mu}$,w are matrices in $M_{N}(\mathbb{C})$ with $u(x)$ positive and invertible.

For a smooth section $a \in \Gamma(\operatorname{End}(V))$, the existence of an asymptotics for the heat-trace $\operatorname{Tr} a e^{-t P} \sim_{t \downarrow 0} \sum_{r=0}^{\infty} a_{r}(a, P) t^{(r-d) / 2}$ is known (see $[11,12]$ ), with coefficients given by $a_{r}(a, P)=\int_{M} \operatorname{dvol}_{g}(x) a_{r}(a, P)(x)$, where $\operatorname{dvol}_{g}(x):=|g|^{1 / 2} \mathrm{~d} x$ and $|g|:=\operatorname{det}\left(g_{\mu \nu}\right)$; more precisely given by

$$
a_{r}(a, P)(x):=\operatorname{tr}\left[a(x) \mathscr{R}_{r}(x)\right]
$$

where tr is the trace on $M_{N}(\mathbb{C})$, and $\mathscr{R}_{r}$ is a (local) section of End $(V)$.

The explicit knowledge of the $a_{r}$ and $\mathscr{R}_{r}$ is important both in mathematics and physics, and several attempts can be found in the literature for many classes of operators $P$, see the books $[4,7,10,18]$. While here we extend a previous method $[13,14]$, this paper is actually self-contained.

To start with, it is convenient to use a covariant derivative $\nabla$ on $V$ and to parameterize the differential operator $P$ as:

$$
P:=-\left(|g|^{-1 / 2} \nabla_{\mu}|g|^{1 / 2} g^{\mu v} u \nabla_{v}+p^{\mu} \nabla_{\mu}+q\right)=-g^{\mu v} u \nabla_{\mu} \nabla_{v}-\left(p^{v}+g^{\mu v}\left(\nabla_{\mu} u\right)-\Gamma^{v} u\right) \nabla_{v}-q,
$$

where $u, p^{\mu}, q$ are sections of $\operatorname{End}(V)$ (see [13, Appendix A.4] for the swap between $\left(v^{\mu}, w\right)$ and $\left(p^{\mu}, q\right)$ ) and

$$
\Gamma^{v}:=g^{\mu \rho} \Gamma_{\mu \rho}^{v} \text { where the } \Gamma_{\mu \rho}^{v} \text { are the Christoffel symbols of } g .
$$

The computation of $\mathscr{R}_{r}$ is realized through $\operatorname{Tr} a e^{-t P}=\int \mathrm{d} x \operatorname{tr}[a(x) \mathscr{K}(t, x, x)]$, where $\mathscr{K}(t, x, x)$ is the diagonal of the kernel of $e^{-t P}$ defined for any section $s \in \Gamma(V)$ by $\int_{M} \mathrm{~d} y \mathscr{K}(t, x, y) s(y)=\left(e^{-t P} s\right)(x)$. Recall that this kernel can be computed using a compactly supported section $s$ with support in a open subset $U$ of $M$ which gives at the same time a chart of $M$ and a trivialization of $V$ (and $\operatorname{End}(V)$ ). In that situation, we can look at $s$ as a map $s: U \rightarrow \mathbb{C}^{N}$.

The use of the Fourier transform $\widehat{s}(\xi):=(2 \pi)^{-d / 2} \int_{U} \mathrm{~d} y e^{-i y \cdot \xi} s(y)$ of $s$ with inverse $s(x)=(2 \pi)^{-d / 2} \int_{\mathbb{R}^{d}} \mathrm{~d} \xi e^{i x \cdot \xi} \widehat{s}(\xi)$ yields to

$$
\left(e^{-t P} s\right)(x)=(2 \pi)^{-d / 2} \int_{\mathbb{R}^{d}} \mathrm{~d} \xi\left(e^{-t P} e^{i x \cdot \xi}\right) \widehat{s}(\xi)=(2 \pi)^{-d} \int_{\mathbb{R}^{d}} \mathrm{~d} \xi \int_{U} \mathrm{~d} y e^{-i y \cdot \xi}\left(e^{-t P} e^{i x \cdot \xi}\right) s(y) .
$$

${ }^{1}$ bruno.iochum@cpt.univ-mrs.fr, thierry.masson@cpt.univ-mrs.fr 
For fixed $(x, y) \in M \times M$, we can look at $e^{-i y \cdot \xi}\left(e^{-t P} e^{i x \cdot \xi}\right)$, and then at $\mathscr{K}(t, x, y)=(2 \pi)^{-d} \int_{\mathbb{R}^{d}} \mathrm{~d} \xi e^{-i y \cdot \xi}\left(e^{-t P} e^{i x \cdot \xi}\right)$, as maps $\mathbb{C}^{N} \rightarrow \mathbb{C}^{N}$, because the function $x \mapsto e^{i x \cdot \xi}$ "absorbs" all the derivatives operators in $e^{-t P}$. We now give another expression for the matrix $\mathscr{K}(t, x, y) \in M_{N}(\mathbb{C})$. Since for any $s: U \rightarrow \mathbb{C}^{N}$,

$$
\begin{aligned}
& -\left(P e^{i x \xi} s\right)(x) \\
& \quad=e^{i x \xi}\left(\left[-g^{\mu v} u \xi_{\mu} \xi_{v}+i \xi_{\mu}\left(p^{\mu}+g^{\mu v}\left(\nabla_{v} u\right)-\Gamma^{v} u+2 g^{\mu v} u \nabla_{v}\right)+u \Delta+\left(p^{v}+g^{\mu v}\left(\nabla_{\mu} u\right)-\Gamma^{v} u\right) \nabla_{v}+q\right] s\right)(x) \\
& \quad=-e^{i x \xi}([H+K+P] s)(x)
\end{aligned}
$$

where we have introduced

$$
\begin{aligned}
& \Delta:=-g^{\mu v} \nabla_{\mu} \nabla_{v}, \\
& K:=-i \xi_{\mu}\left(p^{\mu}+g^{\mu v}\left(\nabla_{\nu} u\right)-\Gamma^{\mu} u+2 g^{\mu v} u \nabla_{v}\right), \\
& H:=g^{\mu v} u \xi_{\mu} \xi_{v},
\end{aligned}
$$

one gets $e^{-i y \cdot \xi}\left(e^{-t P} e^{i x \cdot \xi} s\right)(x)=e^{i(x-y) \cdot \xi}\left(e^{-t[H+K+P]} s\right)(x)$.

The operators $H$ and $K$ being essential here, they will be also decomposed as $H=\xi_{\mu} \xi_{v} H^{\mu v}$ and $K=\xi_{\mu} K^{\mu}$ where

$$
\begin{aligned}
& H^{\mu \nu}:=g^{\mu \nu} u, \\
& K^{\mu}:=-i\left(p^{\mu}+g^{\mu \nu}\left(\nabla_{v} u\right)-\Gamma^{\mu} u+2 g^{\mu \nu} u \nabla_{v}\right)=-i\left(L^{\mu}+2 H^{\mu \nu} \nabla_{v}\right), \\
& L^{\mu}:=N^{\mu}-\Gamma^{\mu} u, \\
& N^{\mu}:=p^{\mu}+g^{\mu \nu}\left(\nabla_{v} u\right),
\end{aligned}
$$

and thus

$$
P=-H^{\mu v} \nabla_{\mu} \nabla_{v}-L^{\mu} \nabla_{\mu}-q .
$$

The introduction of the variable $N^{\mu}$ will be justified in Lemma 3.1.

Then, for any $v \in \mathbb{C}^{N}$ and at fixed values $x$ and $y$,

$$
\mathscr{K}(t, x, y) v=(2 \pi)^{-d} \int_{\mathbb{R}^{d}} \mathrm{~d} \xi e^{i(x-y) \cdot \xi} e^{-t[H+K+P]} v=t^{-d / 2}(2 \pi)^{-d} \int_{\mathbb{R}^{d}} \mathrm{~d} \xi e^{i(x-y) \cdot \xi} e^{-H-\sqrt{t} K-t P} v,
$$

and the diagonal part of the kernel is $\mathbb{C}^{N} \ni v \mapsto \mathscr{K}(t, x, x) v=t^{-d / 2}(2 \pi)^{-d} \int_{\mathbb{R}^{d}} \mathrm{~d} \xi e^{-H-\sqrt{t} K-t P} v$.

From now on, $v \in \mathbb{C}^{N}$ will be considered as a locally constant section of $V$.

The computation of this matrix-valued function $\mathscr{K}$ is based on the Volterra series

$$
e^{A+B}=e^{A}+\sum_{k=1}^{\infty} \int_{\Delta_{k}} \mathrm{~d} s e^{\left(1-s_{1}\right) A} B e^{\left(s_{1}-s_{2}\right) A} \cdots e^{\left(s_{k-1}-s_{k}\right) A} B e^{s_{k} A},
$$

where

$$
\Delta_{k}:=\left\{s=\left(s_{1}, \ldots, s_{k}\right) \in \mathbb{R}_{+}^{k} \mid 0 \leq s_{k} \leq s_{k-1} \leq \cdots \leq s_{2} \leq s_{1} \leq 1\right\} \quad \text { (we also use the convention } s_{0}:=1, s_{k+1}:=0 \text { ), }
$$

and by convention $\Delta_{0}:=\varnothing$. This gives, for $A=-H$ and $B=-t^{1 / 2} K-t P$ (omitting the $\xi$-dependence),

$$
e^{-H-\sqrt{t} K-t P} v=e^{-H} v+\sum_{k=1}^{\infty}(-1)^{k} f_{k}\left[(\sqrt{t} K+t P)^{\otimes^{k}}\right] v
$$

where for any $k \in \mathbb{N}$, the map $f_{k}(\xi): M_{N}[\xi, \nabla]^{\otimes^{k}} \rightarrow M_{N}[\xi]$ is defined, for any $v \in \mathbb{C}^{N}$, by

$$
\begin{aligned}
& f_{k}(\xi)\left[B_{1} \otimes \cdots \otimes B_{k}\right] v:=\int_{\Delta_{k}} \mathrm{~d} s e^{\left(s_{1}-s_{0}\right) H(\xi)} B_{1} e^{\left(s_{2}-s_{1}\right) H(\xi)} B_{2} \cdots B_{k} e^{\left(s_{k+1}-s_{k}\right) H(\xi)} v, \\
& f_{0}(\xi)[\lambda] v:=\lambda e^{-H(\xi)} v, \quad \text { for } \lambda \in \mathbb{C}=: M_{N}(\mathbb{C})^{\otimes^{0}} .
\end{aligned}
$$

Here the $B_{i}$ are matrix-valued differential operators in $\nabla_{\mu}$ depending on $x$ and (linearly in) $\xi$, and $\lambda \in \mathbb{C}$. 
Remark 1.1 (Notation and convention) By convention, each $\nabla_{\mu}$ in $B_{i} \in M_{N}[\xi, \nabla]$ acts on all its right remaining terms. Since $v$ is constant, this allows to identify $f_{k}(\xi)\left[B_{1} \otimes \cdots \otimes B_{k}\right]$ as matrix valued functions, $v \mapsto f_{k}(\xi)\left[B_{1} \otimes \cdots \otimes B_{k}\right] v$, even if the $B_{i}$ 's contain derivative operators.

The lemma 4.1 will get rid of these explicit differential operators in the arguments of $f_{k}(\xi)$ and will produce formulas with matrix-valued arguments only. This is an essential result for the method.

The first terms of (1.7) are (omitting again the $\xi$-dependence)

$$
\begin{aligned}
e^{-H-\sqrt{t} K-t P} v= & f_{0}[1] v-t^{1 / 2} f_{1}[K] v+t\left(f_{2}[K \otimes K]-f_{1}[P]\right) v+t^{3 / 2}\left(f_{2}[K \otimes P]+f_{2}[P \otimes K]-f_{3}[K \otimes K \otimes K]\right) v \\
& +t^{2}\left(f_{2}[P \otimes P]-f_{3}[K \otimes K \otimes P]-f_{3}[K \otimes P \otimes K]-f_{3}[P \otimes K \otimes K]+f_{4}[K \otimes K \otimes K \otimes K]\right) v+\mathcal{O}\left(t^{2}\right) .
\end{aligned}
$$

Since the $\xi$-integral cancels the non-integers powers of $t$, one recovers the coefficients $a_{r}$ via the asymptotics behavior $\operatorname{Tr}\left[a e^{-t P}\right] \underset{t \downarrow 0}{\sim} t^{-d / 2} \sum_{r=0}^{\infty} a_{r}(a, P) t^{r / 2}$ : at the point $x$,

$$
\begin{aligned}
& a_{0}(a, P)(x)=\operatorname{tr} \frac{|g|^{-1 / 2}}{(2 \pi)^{d}} a(x) \int_{\mathbb{R}^{d}} \mathrm{~d} \xi f_{0}[1], \\
& a_{2}(a, P)(x)=\operatorname{tr} \frac{|g|^{-1 / 2}}{(2 \pi)^{d}} a(x) \int_{\mathbb{R}^{d}} \mathrm{~d} \xi\left(f_{2}[K \otimes K]-f_{1}[P]\right), \\
& a_{4}(a, P)(x)=\operatorname{tr} \frac{|g|^{-1 / 2}}{(2 \pi)^{d}} a(x) \int_{\mathbb{R}^{d}} \mathrm{~d} \xi\left(f_{2}[P \otimes P]-f_{3}[K \otimes K \otimes P]-f_{3}[K \otimes P \otimes K]-f_{3}[P \otimes K \otimes K]+f_{4}[K \otimes K \otimes K \otimes K]\right) .
\end{aligned}
$$

where the convention of Remark 1.1 is adopted.

While it is tempting to generalize $H^{\mu \nu}=g^{\mu \nu} u$ to an arbitrary strictly positive matrix $H^{\mu \nu}$ (with $H^{\mu \nu}=H^{v \mu}$ ), it is almost impossible to obtain simple expression for the $a_{r}$. For instance the $\xi$-integral of $a_{0}(a, P)(x)$ cannot be done explicitly (see for instance [13] for details and [2, Section 3.3] for a link with Finsler metrics).

The computation of $a_{r}$ goes the following way.

First the calculus of the $\xi$-integral of (1.8) leads, via the spectral decomposition of $H(\xi)=g^{\mu v} u \xi_{\mu} \xi_{v}$, to the universal functions $I_{\alpha, k}:\left(r_{0}, \ldots, r_{k}\right) \in\left(\mathbb{R}_{+}^{*}\right)^{k+1} \mapsto \int_{\Delta_{k}} \mathrm{~d} s\left[\sum_{\ell=0}^{k}\left(s_{\ell}-s_{\ell+1}\right) r_{\ell}\right]^{-\alpha}$, see Section 2.1 or $[13,14]$.

Then, as shown in Section 2.1, the family of functions $I_{\alpha, k}$ satisfies a few relations. Moreover, in the same section, these relations are translated in terms of operators $X_{\alpha, k}$ (see Definition 2.15) acting on the tensor product $B_{1} \otimes \cdots \otimes B_{k}$ of $M_{N}(\mathbb{C})$-valued differential operators and $I_{\alpha, k}$ can be seen as the spectral functions associated to the action of $X_{\alpha, k}$. The differential aspect, which is an important part of the game here, is not a difficulty because the family of $X_{\alpha, k}$ is compatible with derivations, see Proposition 2.11.

The previous described approach and formulae for $a_{r}(a, P)(x)$ are well-known, see especially $[1-3,5,6]$. The originality of this work, compared to previous quoted ones and $[13,14]$, is however to perform the computations using operators instead of spectral functions. One can follow this way more precisely what is the contribution of each term, an information that is a priori lost when adding spectral functions coming from different contributions. These operators, respectively $f_{k}(\xi)$ and $X_{\alpha, k}$, have a universal property since they only depend of a positive invertible matrix-valued which can be either $H(x, \xi)$ or $u(x)$. Thus, in this formal algebraic level, the computations are reduced to a control of the propagation of derivatives inside the arguments, see for instance Proposition 4.1.

However, to secure the results on the matrices $\mathscr{R}_{r}(x)$, we need a covariant formulation of all tools. This is the aim of Section 3 where $P$ is presented in a covariant way in equation (3.7) and, since we know that $\mathscr{R}_{r}(x)$ must then be invariant under a change of coordinates, we choose a normal coordinate system. This implies that the covariant derivative $\nabla$ has to be extended to the total covariant derivative $\widehat{\nabla}$ combining both $\nabla$ and the Levi-Civita connection on $M$. Such an extension requires for the sequel to compute beforehand a few formulae on the action of $\widehat{\nabla}$ on all ingredients.

In particular, in Section 4, we present a formula for the propagation of $\nabla$ within the arguments and show several simplifications due to a few Riemannian contractions which appear all along the computations. This leads to an operational version of the method exposed in Proposition 4.3.

In Section 5 the whole intermediate steps for the calculation of $\mathscr{R}_{2}$ are given and, of course, we recover in this new algebraic setting the previous results of $[13,14]$.

While all computations can be done "by hand" for $\mathscr{R}_{2}$, the case of $\mathscr{R}_{4}$ requires the use of a computer due to the huge number of generated terms. The hard and long part of this work was to develop a code ab initio. The elaboration of such a code is explained in Section 6. It takes care of all intricate aspects of the computations: the algebraic manipulations quoted before, the use of normal coordinates for higher derivatives and last but not least, the simplification of a large number of terms via a reduction process, see (2.24). 
Finally, a formula for $\mathscr{R}_{4}$ is exhibited in Section 7 which is a new result. Of course, it is compatible with old known results like when $u=\mathbb{1}$, see $[10,12]$, but is written here in full generality when the section $u$ is parallel for $\nabla$ and the $N^{v}$ are not zero, while the standard presentation always assumes that $N^{v}=0$ (see Lemma 3.1).

\section{The universal operators $X_{\alpha, k}$}

The aim of this section is to define and study the operators $X_{\alpha, k}$ which depends only on a strictly positive matrix-valued function $h: \mathscr{U} \rightarrow M_{N}(\mathbb{C})$ where $\mathscr{U}$ is a given parameter space. Later on, when the differential operator $P$ will play a role, $h$ will be either $u(x)$ with $\mathscr{U}=U$ or $H(x, \xi)=g^{\mu v}(x) u(x) \xi_{\mu} \xi_{v}$ with $\mathscr{U}=U \times \mathbb{R}^{d}$.

\subsection{The universal spectral functions $I_{\alpha, k}$}

Let us first consider the algebra $\mathscr{A}=M_{N}(\mathbb{C})$ (although many of the theory could be generalized to a unital $C^{*}$-algebra $\mathscr{A}$ ) and $h \in \mathscr{A}$ which is a positive invertible matrix:

$$
0<h \in \mathscr{A}
$$

For any $k \in \mathbb{N}$ and $a_{\ell} \in \mathscr{A}$, let $\left[a_{0}^{R} \otimes a_{1}^{R} \otimes \cdots \otimes a_{k}^{R}\right] \in \mathscr{B}\left(\mathscr{A}^{\otimes^{k+1}}, \mathscr{A}^{\otimes^{k+1}}\right)$ (bounded operator from $\mathscr{A}^{\otimes^{k+1}}$ into itself) be defined by $\left[a_{0}^{R} \otimes a_{1}^{R} \otimes \cdots \otimes a_{k}^{R}\right]\left[b_{0} \otimes \cdots \otimes b_{k}\right]:=b_{0} a_{0} \otimes b_{1} a_{1} \otimes \cdots \otimes b_{k} a_{k}$. For some reason we want to apply such operators to $\mathscr{A}^{\otimes^{k}}$ and to do so, we need the injection of $\mathscr{A}^{\otimes^{k}}$ into $\mathscr{A}^{\otimes^{k+1}}$ :

$$
\kappa_{k}: b_{1} \otimes \cdots \otimes b_{k} \mapsto \mathbb{1} \otimes b_{1} \otimes \cdots \otimes b_{k}, \text { for } k \geq 1, \quad \text { and } \quad \kappa_{0}(\lambda):=\lambda \mathbb{1}, \lambda \in \mathscr{A}^{0}:=\mathbb{C} .
$$

where $\mathbb{1}$ is the unit of $\mathscr{A}$. Now we define the operator $\left(a_{0}^{R} \otimes a_{1}^{R} \otimes \cdots \otimes a_{k}^{R}\right) \in \mathscr{B}\left(\mathscr{A}^{\otimes^{k}}, \mathscr{A}^{\otimes^{k+1}}\right)$ as

$$
\left(a_{0}^{R} \otimes a_{1}^{R} \otimes \cdots \otimes a_{k}^{R}\right):=\left[a_{0}^{R} \otimes a_{1}^{R} \otimes \cdots \otimes a_{k}^{R}\right] \circ \kappa_{k} .
$$

We also need, for $0 \leq \ell \leq k$, the following family of operators:

$$
R_{\ell}(a):=\left(\mathbb{1} \otimes \cdots \otimes \mathbb{1} \otimes a^{R} \otimes \mathbb{1} \otimes \cdots \otimes \mathbb{1}\right) \in \mathscr{B}\left(\mathscr{A}^{\otimes^{k}}, \mathscr{A}^{\otimes^{k+1}}\right) \text { where } a^{R} \text { is at the } \ell \text {-th place. }
$$

For any $k$, denote by $\mathbf{m}: \mathscr{A}^{\otimes^{k+1}} \rightarrow \mathscr{A}$ the multiplication in $\mathscr{A}$. Then $\mathbf{m} \circ\left(a_{0}^{R} \otimes a_{1}^{R} \otimes \cdots \otimes a_{k}^{R}\right) \in \mathscr{B}\left(\mathscr{A}^{\otimes^{k}}, \mathscr{A}\right)$ is given by

$$
\left(\mathbf{m} \circ\left(a_{0}^{R} \otimes a_{1}^{R} \otimes \cdots \otimes a_{k}^{R}\right)\right)\left[b_{1} \otimes \cdots \otimes b_{k}\right]=a_{0} b_{1} a_{1} \cdots b_{k} a_{k} .
$$

We now consider the functional calculus on $h$, with the shorthand notation

$$
E_{\ell}:=E_{r_{\ell}} \text { is the spectral projection associated to the spectral value } r_{\ell} \text { of } h \text {. }
$$

Keep in mind that $\ell$ is not the index of a spectral value but is the index of the position in the $(k+1)$-tensor product. The need to compute the $\xi$-integrals of the operators $f_{k}(\xi)$ drives us to

$$
\int \mathrm{d} \xi \xi_{\mu_{1}} \cdots \xi_{\mu_{2 p}} f_{k}(\xi)=\mathbf{m} \circ \int_{\Delta_{k}} \mathrm{~d} s \int \mathrm{d} \xi \xi_{\mu_{1}} \cdots \xi_{\mu_{2 p}} e^{-C_{k}(s, H(\xi))}=c_{k, \mu_{1} \ldots \mu_{2 p}} \mathbf{m} \circ \int_{\Delta_{k}} \mathrm{~d} s C_{k}(s, u)^{-(d / 2+p)}
$$

where $C_{k}(s, a):=\sum_{\ell=0}^{k}\left(s_{\ell}-s_{\ell+1}\right) R_{\ell}(a)$, permuting the integrals in the first equality and using a Gaussian integration with spherical coordinates in the second one, see [13, Eq. (4.4)]. Thus the functional calculus for $h$ naturally leads us to the following functions (when $\alpha=d / 2+p$ ):

Definition 2.1 For any $\alpha \in \mathbb{R}$ and $k \in \mathbb{N}$, let $I_{\alpha, k}:\left(\mathbb{R}_{+}^{*}\right)^{k+1} \rightarrow \mathbb{R}_{+}$defined by

$$
\begin{aligned}
& I_{\alpha, k}\left(r_{0}, \ldots, r_{k}\right):=\int_{\Delta_{k}} d s\left[\sum_{\ell=0}^{k}\left(s_{\ell}-s_{\ell+1}\right) r_{\ell}\right]^{-\alpha}=\int_{\Delta_{k}} d s\left[\sum_{\ell=0}^{k}\left(s_{\ell}\left(r_{\ell}-r_{\ell-1}\right)\right]^{-\alpha} \quad \text { (with the convention } r_{-1}:=0\right) \\
& I_{\alpha, 0}\left(r_{0}\right):=r_{0}^{-\alpha} \text { for } \alpha \neq 0 .
\end{aligned}
$$


For instance,

$$
\begin{aligned}
& I_{\alpha, k}(\underbrace{r_{0}, \ldots, r_{0}}_{k+1})=\frac{1}{k !} r_{0}^{-\alpha}, \\
& I_{\alpha, 1}\left(r_{0}, r_{1}\right)= \begin{cases}\frac{1}{1-\alpha} \frac{r_{0}^{1-\alpha}-r_{1}^{1-\alpha}}{r_{0}-r_{1}} & \text { for } \alpha \neq 1, \\
\frac{\log \left(r_{0}\right)-\log \left(r_{1}\right)}{r_{0}-r_{1}} & \text { for } \alpha=1,\end{cases}
\end{aligned}
$$

and see $[13,14]$ for other explicit expressions for these integrals.

We will need the following recursion formulas on the functions $I_{\alpha, k}$, seen as generalizations of [13, eq. (3.1)]:

Lemma 2.2 For any $\alpha \in \mathbb{R}$ and $k \in \mathbb{N}$ the family of functions $I_{\alpha, k}$ satisfies

i) $I_{\alpha, k}\left(r_{0}, \ldots, r_{k}\right)=r_{0} I_{\alpha+1, k+1}\left(r_{0}, r_{0}, \ldots, r_{k}\right)+r_{1} I_{\alpha+1, k+1}\left(r_{0}, r_{1}, r_{1}, \ldots, r_{k}\right)+\cdots+r_{k} I_{\alpha+1, k+1}\left(r_{0}, r_{1}, \ldots, r_{k}, r_{k}\right)$.

ii) Moreover, for any $\alpha \neq 1, k, \ell \in \mathbb{N}^{*}$ and $1 \leq \ell \leq k$,

$$
I_{\alpha, k}\left(r_{0}, \ldots, r_{k}\right)=\frac{1}{1-\alpha} \frac{1}{r_{\ell}-r_{\ell-1}}\left[I_{\alpha-1, k-1}\left(r_{0}, \ldots, \widehat{r_{\ell-1}}, \ldots, r_{k}\right)-I_{\alpha-1, k-1}\left(r_{0}, \ldots, \widehat{r_{\ell}}, \ldots, r_{k}\right)\right] .
$$

Proof i) For $0 \leq \ell \leq k$, the integrand in the defining integral of $I_{\alpha+1, k+1}\left(r_{0}, \ldots, r_{\ell}, r_{\ell}, \ldots, r_{k}\right)$ reduces to

$$
\begin{cases}{\left[\left(s_{0}-s_{2}\right) r_{0}+\left(s_{2}-s_{3}\right) r_{1}+\cdots+s_{k+1} r_{k}\right]^{-(\alpha+1)}} & \text { for } \ell=0, \\ {\left[\left(s_{0}-s_{1}\right) r_{0}+\cdots+\left(s_{\ell}-s_{\ell+2}\right) r_{\ell}+\cdots+s_{k+1} r_{k}\right]^{-(\alpha+1)}} & \text { for } 0<\ell<k, \\ {\left[\left(s_{0}-s_{1}\right) r_{0}+\cdots+\left(s_{k-1}-s_{k}\right) r_{k-1}+s_{k} r_{k}\right]^{-(\alpha+1)}} & \text { for } \ell=k,\end{cases}
$$

and then does not depend anymore of the variable $s_{\ell+1}$. Using

$$
\int_{\Delta_{k+1}} \mathrm{~d} s= \begin{cases}\int_{0}^{1} \mathrm{~d} s_{2} \cdots \int_{0}^{s_{k}} \mathrm{~d} s_{k+1} \int_{s_{2}}^{1} \mathrm{~d} s_{1} & \text { for } \ell=0, \\ \int_{0}^{1} \mathrm{~d} s_{1} \cdots \int_{0}^{s_{\ell-1}} \mathrm{~d} s_{\ell} \int_{0}^{s_{\ell}} \mathrm{d} s_{\ell+2} \int_{0}^{s_{\ell+2}} \mathrm{~d} s_{\ell+3} \cdots \int_{0}^{s_{k}} \mathrm{~d} s_{k+1} \int_{s_{\ell+2}}^{s_{\ell}} \mathrm{d} s_{\ell+1} & \text { for } 0<\ell<k, \\ \int_{0}^{1} \mathrm{~d} s_{1} \int_{0}^{s_{1}} \mathrm{~d} s_{2} \cdots \int_{0}^{s_{k}} \mathrm{~d} s_{k+1} & \text { for } \ell=k,\end{cases}
$$

the integration along $s_{\ell+1}$ produces, respectively, the factors $\left(1-s_{2}\right),\left(s_{\ell}-s_{\ell+2}\right)$, and $s_{k}$.

Let us perform the change of variables

$$
\begin{cases}\left(s_{2}, s_{3}, \ldots, s_{k+1}\right) \rightarrow\left(s_{1}, s_{2}, \ldots, s_{k}\right) & \text { for } \ell=0, \\ \left(s_{1}, s_{2}, \ldots, s_{\ell}, s_{\ell+2}, \ldots, s_{k+1}\right) \rightarrow\left(s_{1}, s_{2}, \ldots, s_{\ell}, s_{\ell+1}, \ldots, s_{k}\right) & \text { for } 0<\ell<k .\end{cases}
$$

There is no need to change the variables for $\ell=k$. Then, for all $0 \leq \ell \leq k$, the remaining integration is over $\Delta_{k}$ and all the brackets in (2.9) coincide (with the case $\ell=k$ ). This implies that the RHS of (2.7) can be recombined, through the factorization of this common bracket, as a single integral

$$
\int_{\Delta_{k}} \mathrm{~d} s\left[\left(s_{0}-s_{1}\right) r_{0}+\cdots+\left(s_{\ell}-s_{\ell+1}\right) r_{\ell}+\cdots+\left(s_{k}-s_{k+1}\right) r_{k}\right]\left[\left(s_{0}-s_{1}\right) r_{0}+\cdots+\left(s_{k}-s_{k+1}\right) r_{k}\right]^{-(\alpha+1)},
$$

which is nothing else but $I_{\alpha, k}\left(r_{0}, \ldots, r_{k}\right)$.

ii) For any $\ell, 1 \leq \ell \leq k$, one has $\int_{\Delta_{k}} \mathrm{~d} s=\int_{0}^{1} \mathrm{~d} s_{1} \int_{0}^{s_{1}} \mathrm{~d} s_{2} \cdots \int_{0}^{s_{\ell-2}} \mathrm{~d} s_{\ell-1} \int_{0}^{s_{\ell-1}} \mathrm{~d} s_{\ell+1} \int_{0}^{s_{\ell+1}} \mathrm{~d} s_{\ell+2} \cdots \int_{0}^{s_{k-1}} \mathrm{~d} s_{k} \int_{s_{\ell+1}}^{s_{\ell-1}} \mathrm{~d} s_{\ell}$. Performing the last integration along $s_{\ell}$, one gets

$$
\begin{aligned}
\int_{s_{\ell+1}}^{s_{\ell-1}} \mathrm{~d} s_{\ell}\left[r_{0}+s_{1}\left(r_{1}-r_{0}\right)+\right. & \left.\cdots+s_{k}\left(r_{k}-r_{k-1}\right)\right]^{-\alpha} \\
= & \frac{1}{1-\alpha} \frac{1}{r_{\ell}-r_{\ell-1}}\left[\left[r_{0}+s_{1}\left(r_{1}-r_{0}\right)+\cdots+s_{k}\left(r_{k}-r_{k-1}\right)\right]^{1-\alpha}\right]_{s_{\ell}=s_{\ell+1}}^{s_{\ell}=s_{\ell-1}} \\
= & \frac{1}{1-\alpha} \frac{1}{r_{\ell}-r_{\ell-1}}\left[r_{0}+\cdots+s_{\ell-1}\left(r_{\ell}-r_{\ell-2}\right)+s_{\ell+1}\left(r_{\ell+1}-r_{\ell}\right)+\cdots+s_{k}\left(r_{k}-r_{k-1}\right)\right]^{1-\alpha} \\
& \quad-\frac{1}{1-\alpha} \frac{1}{r_{\ell}-r_{\ell-1}}\left[r_{0}+\cdots+s_{\ell-1}\left(r_{\ell-1}-r_{\ell-2}\right)+s_{\ell+1}\left(r_{\ell+1}-r_{\ell-1}\right)+\cdots+s_{k}\left(r_{k}-r_{k-1}\right)\right]^{1-\alpha} .
\end{aligned}
$$

An integration along the other variables $s_{i}$ 's gives the equation (2.8). 


\subsection{Definitions and properties of the operators $X_{\alpha, k}$}

As before, $h$ is a positive invertible element in $\mathscr{A}=M_{N}(\mathbb{C})$.

Definition 2.3 For any $k \in \mathbb{N}$, given a function $f:\left(\mathbb{R}_{+}^{*}\right)^{k+1} \rightarrow \mathbb{C}$, we define the operator $\pi_{f}$ acting on $\mathscr{A}^{\otimes^{k}}$ as

$$
\pi_{f}:=f\left(r_{0}, \ldots, r_{k}\right) \mathbf{m} \circ\left(E_{0}^{R} \otimes \cdots \otimes E_{k}^{R}\right) \in \mathscr{B}\left(\mathscr{A}^{\otimes^{k}}, \mathscr{A}\right),
$$

with an (implicit) summation over $k+1$-tuples $\left(r_{0}, \ldots, r_{k}\right)$ of spectral values of $h$.

In particular, using (2.2) for $\lambda \in \mathbb{C}, b_{\ell} \in \mathscr{A}$,

$$
\begin{aligned}
& \pi_{f}[\lambda]=f\left(r_{0}\right) \lambda E_{0}=\lambda f(h) \in \mathscr{A} \text { for } k=0, \\
& \pi_{f}\left[b_{1} \otimes \cdots \otimes b_{k}\right]=f\left(r_{0}, \ldots, r_{k}\right) E_{0} b_{1} E_{1} \cdots E_{k-1} b_{k} E_{k} \in \mathscr{A} \text { for } k \in \mathbb{N}^{*} .
\end{aligned}
$$

If $\widehat{f}\left(r_{0}, \ldots, r_{k}\right):=\int_{Z} \mathrm{~d} z f\left(z ; r_{0}, \ldots r_{k}\right)$, where $Z$ is a measured space, then

$$
\pi_{\widehat{f}}\left[b_{1} \otimes \cdots \otimes b_{k}\right]=\int_{Z} \mathrm{~d} z \pi_{f(z)}\left[b_{1} \otimes \cdots \otimes b_{k}\right]
$$

since the implicit summation over $r_{0}, \ldots, r_{k}$ is finite and $E_{0} b_{1} E_{1} \cdots E_{k-1} b_{k} E_{k}$ is z-independent.

The spectral function $f$ in the equation (2.12) has a peculiar modification if one of the variables $b_{\ell}$ is a function of $h$ : Lemma 2.4 For any continuous function $F: \mathbb{R}_{+}^{*} \rightarrow \mathbb{C}$ and $1 \leq \ell \leq k$, we have

$$
\begin{aligned}
& \pi_{f}\left[b_{1} \otimes \cdots \otimes b_{\ell-1} \otimes F(h) \otimes b_{\ell+1} \otimes \cdots \otimes b_{k}\right]=\pi_{\tilde{f}}\left[b_{1} \otimes \cdots \otimes b_{\ell-1} \otimes b_{\ell+1} \otimes \cdots \otimes b_{k}\right], \\
& \text { with } \tilde{f}\left(r_{0}, \ldots, r_{k-1}\right):=F\left(r_{\ell-1}\right) f\left(r_{0}, \ldots, r_{\ell-1}, r_{\ell-1}, r_{\ell}, \ldots, r_{k-1}\right) .
\end{aligned}
$$

Proof Since $E_{\ell-1} F(h) E_{\ell}=F\left(r_{\ell-1}\right) E_{\ell-1} E_{\ell}=\delta_{r_{\ell}, r_{\ell-1}} F\left(r_{\ell-1}\right) E_{\ell-1}$, we get

$$
\begin{aligned}
\pi_{f}\left[b_{1} \otimes \cdots\right. & \left.\otimes b_{\ell-1} \otimes F(h) \otimes b_{\ell+1} \otimes \cdots \otimes b_{k}\right] \\
& =f\left(r_{0}, \ldots, r_{\ell-1}, r_{\ell}, r_{\ell+1}, \ldots, r_{k}\right) E_{0} b_{1} E_{1} \cdots b_{\ell-1} E_{\ell-1} F(h) E_{\ell} b_{\ell+1} E_{\ell+1} \cdots b_{k} E_{k} \\
& =F\left(r_{\ell-1}\right) f\left(r_{0}, \ldots, r_{\ell-1}, r_{\ell-1}, \ldots, r_{k}\right) E_{0} b_{1} E_{1} \cdots b_{\ell-1} E_{\ell-1} b_{\ell+1} E_{\ell+1} \cdots b_{k} E_{k},
\end{aligned}
$$

which, after a relabeling of the summation indices $i \rightarrow i-1$ for $i>\ell$, can be written as in (2.14).

Since this result will be widely used, let us give an example: for $a, b \in \mathscr{A}$ and $f:\left(\mathbb{R}_{+}^{*}\right)^{5} \rightarrow \mathbb{C}$

$$
\pi_{f}\left[h^{2} \otimes a \otimes h^{1 / 2} \otimes b\right]=r_{0}^{2} r_{1}^{1 / 2} f\left(r_{0}, r_{0}, r_{1}, r_{1}, r_{2}\right) E_{0} a E_{1} b E_{2}
$$

An important case of operators $\pi_{f}$ is the family of operators $X_{\alpha, k}$ associated to the universal functions $I_{\alpha, k}$ (see Definition 2.1) and to $h$, which will play a crucial role in the sequel precisely because of their universality.

$$
X_{\alpha, k}:=\pi_{I_{\alpha, k}} \text { for } k \in \mathbb{N} \text {. }
$$

Again, for brevity of notation on the use of $X_{\alpha, k}$, both the $h$-dependence and the summation when applied to arguments will be implicit.

From the equations (2.5) and (2.4) we immediately check that

$$
X_{\alpha, k}[\underbrace{h \otimes \cdots \otimes h}_{k}]=\frac{1}{k !} h^{-\alpha}=X_{\alpha, 0}[h] .
$$

We also remark that for any matrices $b_{i}, c$ in $\mathscr{A}$ such that $[c, h]=0$, we have the two following factorizations:

$$
\begin{aligned}
& X_{\alpha, k}\left[b_{1} \otimes \cdots \otimes b_{\ell-1} \otimes b_{\ell} c \otimes b_{\ell+1} \otimes \cdots \otimes b_{k}\right]=X_{\alpha, k}\left[b_{1} \otimes \cdots \otimes b_{k}\right] c, \quad \text { if }\left[c, b_{i}\right]=0 \text { for } \ell+1 \leq i \leq k, \\
& X_{\alpha, k}\left[b_{1} \otimes \cdots \otimes b_{\ell-1} \otimes c b_{\ell} \otimes b_{\ell+1} \otimes \cdots \otimes b_{k}\right]=c X_{\alpha, k}\left[b_{1} \otimes \cdots \otimes b_{k}\right], \quad \text { if }\left[c, b_{i}\right]=0 \text { for } 1 \leq i \leq \ell-1 .
\end{aligned}
$$

For $a \in \mathscr{A}$ and $\ell=1, \ldots, k$, let $i_{a}^{(\ell)}: \mathscr{B}\left(\mathscr{A}^{\otimes^{k}}, \mathscr{A}\right) \rightarrow \mathscr{B}\left(\mathscr{A}^{\otimes^{k-1}}, \mathscr{A}\right)$ be defined by

$$
\left(i_{a}^{(\ell)} \mathbb{B}\right)\left[b_{1} \otimes \cdots \otimes b_{k-1}\right]:=\mathbb{B}\left[b_{1} \otimes \cdots \otimes b_{\ell-1} \otimes a \otimes b_{\ell} \otimes \cdots \otimes b_{k-1}\right],
$$

which inserts $a$ at the $\ell$-th place in $\mathbb{B}$. For instance, one easily checks that

$$
i_{a}^{(\ell)} \mathbf{m} \circ\left(a_{0}^{R} \otimes a_{1}^{R} \otimes \cdots \otimes a_{k}^{R}\right)=\mathbf{m} \circ\left(a_{0}^{R} \otimes a_{1}^{R} \otimes \cdots \otimes\left[a_{\ell-1} a a_{\ell}\right]^{R} \otimes \cdots \otimes a_{k}^{R}\right) \in \mathscr{B}\left(\mathscr{A}^{\otimes^{k-1}}, \mathscr{A}\right) .
$$


Lemma 2.5 The operators $X_{\alpha, k}$ satisfy $X_{\alpha, k}=\sum_{\ell=1}^{k+1} i_{h}^{(\ell)} X_{\alpha+1, k+1}$.

More explicitly, the following expansion holds true for any $b_{\ell} \in \mathscr{A}$ :

$$
\begin{aligned}
& X_{\alpha, k}\left[b_{1} \otimes \cdots \otimes b_{k}\right]= \\
& \quad X_{\alpha+1, k+1}\left[h \otimes b_{1} \otimes \cdots \otimes b_{k}\right]+X_{\alpha+1, k+1}\left[b_{1} \otimes h \otimes \cdots \otimes b_{k}\right]+\cdots+X_{\alpha+1, k+1}\left[b_{1} \otimes \cdots \otimes b_{k} \otimes h\right] .
\end{aligned}
$$

PROOF This follows from equation (2.7).

Corollary 2.6 For any $a, b \in \mathscr{A}$ and any $n \geq 1$, one has

$$
\begin{aligned}
X_{\alpha, 1}[a] & =n ! \sum_{\substack{\ell_{1}, \ell_{2} \geq 0 \\
\ell_{1}+\ell_{2}=n}} X_{\alpha, n+1}[\underbrace{h \otimes \cdots \otimes h}_{\ell_{1} \text { times }} \otimes a \otimes \underbrace{h \otimes \cdots \otimes h}_{\ell_{2} \text { times }}], \\
X_{\alpha, 2}[a \otimes b] & =n ! \sum_{\substack{\ell_{1}, \ell_{2}, \ell_{3} \geq 0 \\
\ell_{1}+\ell_{2}+\ell_{3}=n}}^{h \otimes} X_{\alpha, n+1}[\underbrace{h \otimes \cdots \otimes h}_{\ell_{1} \text { times }} \otimes a \otimes \underbrace{h \otimes \cdots \otimes h}_{\ell_{2} \text { times }} \otimes b \otimes \underbrace{h \otimes \cdots \otimes h}_{\ell_{3} \text { times }}] .
\end{aligned}
$$

PROOF We prove the first relation (2.21) by induction. When $n=1$, the use of Lemma 2.5 yields to the desired relation: $X_{\alpha, 1}[a]=X_{\alpha, 2}[h \otimes a]+X_{\alpha, 2}[a \otimes h]$. Assuming the relation holds for $n \geq 1$, then

$$
\begin{aligned}
& X_{\alpha, 1}[a]=n ! \sum_{\substack{\ell_{1}, \ell_{2} \geq 0 \\
\ell_{1}+\ell_{2}=n}} X_{\alpha, n+1}[\underbrace{h \otimes \cdots \otimes h}_{\ell_{1} \text { times }} \otimes a \otimes \underbrace{h \otimes \cdots \otimes h}_{\ell_{2} \text { times }}], \\
& =n ! \sum_{\substack{\ell_{1}, \ell_{2} \geq 0 \\
\ell_{1}+\ell_{2}=n}}\left[\left(\ell_{1}+1\right) X_{\alpha, n+2}[\underbrace{h \otimes \cdots \otimes h}_{\ell_{1}+1 \text { times }} \otimes a \otimes \underbrace{h \otimes \cdots \otimes h}_{\ell_{2} \text { times }}]+\left(\ell_{2}+1\right) X_{\alpha, n+2}[\underbrace{h \otimes \cdots \otimes h}_{\ell_{1} \text { times }} \otimes a \otimes \underbrace{h \otimes \cdots \otimes h}_{\ell_{2}+1 \text { times }}]\right] \\
& =n ! \sum_{\substack{\ell_{1} \geq 1, \ell_{2} \geq 0 \\
\ell_{1}+\ell_{2}=n+1}} \ell_{1} X_{\alpha, n+2}[\underbrace{h \otimes \cdots \otimes h}_{\ell_{1} \text { times }} \otimes a \otimes \underbrace{h \otimes \cdots \otimes h}_{\ell_{2} \text { times }}]+n ! \sum_{\substack{\ell_{1} \geq 0, \ell_{2} \geq 1 \\
\ell_{1}+\ell_{2}=n+1}} \ell_{2} X_{\alpha, n+2}[\underbrace{h \otimes \cdots \otimes h}_{\ell_{1} \text { times }} \otimes a \otimes \underbrace{h \otimes \cdots \otimes}_{\ell_{2} \text { times }}]
\end{aligned}
$$

after changes of summation parameters $\ell_{1}+1 \rightarrow \ell_{1}$ and $\ell_{2}+1 \rightarrow \ell_{2}$ in the two sums.

Then, extending the summation ranges with $\ell_{1}=0$ and $\ell_{2}=0$ since they do not contribute, one gets

$$
\begin{aligned}
X_{\alpha, 1}[a] & =n ! \sum_{\substack{\ell_{1}, \ell_{2} \geq 0 \\
\ell_{1}+\ell_{2}=n+1}}\left(\ell_{1}+\ell_{2}\right) X_{\alpha, n+2}[\underbrace{h \otimes \cdots \otimes h}_{\ell_{1} \text { times }} \otimes a \otimes \underbrace{h \otimes \cdots \otimes h}_{\ell_{2} \text { times }}] \\
& =(n+1) ! \sum_{\substack{\ell_{1}, \ell_{2} \geq 0 \\
\ell_{1}+\ell_{2}=n+1}}^{h X_{\alpha, n+2}}[\underbrace{h \otimes \cdots \otimes h}_{\ell_{1} \text { times }} \otimes a \otimes \underbrace{h \otimes \cdots \otimes h}_{\ell_{2} \text { times }}]
\end{aligned}
$$

and (2.21) holds true. The relation (2.21) is proved similarly.

We will also use the notion of expansion: for $k \in \mathbb{N}^{*}, \mathscr{E}_{k}: \mathscr{A}^{\otimes^{k}} \rightarrow \mathscr{A}^{\otimes^{k+1}}$ is defined for $b_{\ell} \in \mathscr{A}$ by

$$
\mathscr{E}_{k}\left[b_{1} \otimes \cdots \otimes b_{k}\right]:=h \otimes b_{1} \otimes \cdots \otimes b_{k}+b_{1} \otimes h \otimes \cdots \otimes b_{k}+\cdots+b_{1} \otimes \cdots \otimes b_{k} \otimes h,
$$

and the previous lemma can be read as:

$$
X_{\alpha+1, k+1} \circ \mathscr{E}_{k}=X_{\alpha, k}, \quad \forall k \in \mathbb{N}^{*}
$$

or seen as a reduction process after the expansion $\mathscr{E}_{k}$.

Lemma 2.7 Assume $\alpha \neq 1$.

i) For any $b \in \mathscr{A}$,

$$
X_{\alpha, 1}[[b, h]]=\frac{1}{1-\alpha}\left[b, h^{1-\alpha}\right] .
$$

ii) For any $2 \leq k, \ell \in \mathbb{N}, \ell<k$, and $b_{i} \in \mathscr{A}$,

$$
\begin{array}{r}
X_{\alpha, k}\left[b_{1} \otimes \cdots \otimes b_{\ell-1} \otimes\left[b_{\ell}, h\right] \otimes b_{\ell+1} \otimes \cdots \otimes b_{k}\right]=\frac{1}{1-\alpha}( \\
+X_{\alpha-1, k-1}\left[b_{1} \otimes \cdots \otimes b_{\ell-2} \otimes b_{\ell-1} b_{\ell} \otimes b_{\ell+1} \otimes \cdots \otimes b_{k}\right] \\
\left.-X_{\alpha-1, k-1}\left[b_{1} \otimes \cdots \otimes b_{\ell-1} \otimes b_{\ell} b_{\ell+1} \otimes \otimes b_{\ell+2} \cdots \otimes b_{k}\right]\right) .
\end{array}
$$

iii) For any $k \geq 2$ and any $b_{i} \in \mathscr{A}$,

$$
X_{\alpha, k}\left[b_{1} \otimes \cdots \otimes b_{k-1} \otimes\left[b_{k}, h\right]\right]=\frac{1}{1-\alpha}\left(+X_{\alpha-1, k-1}\left[b_{1} \otimes \cdots \otimes b_{k-2} \otimes b_{k-1} b_{k}\right]-X_{\alpha-1, k-1}\left[b_{1} \otimes \cdots \otimes b_{k-1}\right] b_{k}\right) .
$$


Proof i) Thanks to the definition (2.15), (2.8) and (2.6), we have

$$
X_{\alpha, 1}[[b, h]]=I_{\alpha, 1}\left(r_{0}, r_{1}\right) E_{0}(b h-h b) E_{1}=\left(r_{1}-r_{0}\right) \frac{r_{1}^{1-\alpha}-r_{0}^{1-\alpha}}{(1-\alpha)\left(r_{1}-r_{0}\right)} E_{0} b E_{1}=\frac{1}{1-\alpha}\left(b h^{1-\alpha}-h^{1-\alpha} b\right)
$$

because the implicit summation over $r_{0}$ (resp. $r_{1}$ ) of $E_{0}$ (resp. $E_{1}$ ) gives $\mathbb{1}$.

ii) The LHS of (2.26) is equal to

$$
\begin{aligned}
& \left(r_{\ell}-r_{\ell-1}\right) I_{\alpha, k}\left(r_{0}, \ldots, r_{k}\right) E_{0} b_{1} \cdots E_{\ell-2} b_{\ell-1} E_{\ell-1} b_{\ell} E_{\ell} \cdots E_{k-1} b_{k} E k \\
& =\frac{1}{1-\alpha}\left[I_{\alpha-1, k-1}\left(r_{0}, \ldots, \widehat{r_{\ell-1}}, \ldots, r_{k}\right) E_{0} b_{1} \cdots b_{\ell-1} E_{\ell-1} b_{\ell} E_{\ell} b_{\ell+1} \cdots E_{k-1} b_{k} E k\right. \\
& \left.\quad-I_{\alpha-1, k-1}\left(r_{0}, \ldots, \widehat{r}_{\ell}, \ldots, r_{k}\right) E_{0} b_{1} \cdots b_{\ell-1} E_{\ell-1} b_{\ell} E_{\ell} b_{\ell+1} \cdots E_{k-1} b_{k} E k\right] \\
& =\frac{1}{1-\alpha}\left(X_{\alpha-1, k-1}\left[b_{1} \otimes \cdots \otimes b_{\ell-2} \otimes b_{\ell-1} b_{\ell} \otimes b_{\ell+1} \otimes \cdots \otimes b_{k}\right]\right. \\
& \left.\quad \quad-X_{\alpha-1, k-1}\left[b_{1} \otimes \cdots \otimes b_{\ell-1} \otimes b_{\ell} b_{\ell+1} \otimes \otimes b_{\ell+2} \cdots \otimes b_{k}\right]\right)
\end{aligned}
$$

because the missing summations in $r_{\ell-1}$ and $r_{\ell}$ implies that $E_{\ell-1}$ and $E_{\ell}$ are replaced by $\mathbb{1}$.

iii) Similarly, the LHS of (2.27) is equal to

$$
\begin{aligned}
\left(r_{k}\right. & \left.\left.-r_{k-1}\right)\right) I_{\alpha, k}\left(r_{0}, \ldots, r_{k}\right) E_{0} b_{1} \cdots b_{k-1} E_{k-1} b_{k} E k \\
& =\frac{1}{1-\alpha}\left[I_{\alpha-1, k-1}\left(r_{0}, \ldots, \widehat{r_{k-1}}, \ldots, r_{k}\right) E_{0} b_{1} \cdots b_{k-1} E_{k-1} b_{k} E_{k}-I_{\alpha-1, k-1}\left(r_{0}, \ldots, \widehat{r_{k}}, \ldots, r_{k}\right) E_{0} b_{1} \cdots b_{k-1} E_{k-1} b_{k} E_{k}\right] \\
& =\frac{1}{1-\alpha}\left(X_{\alpha-1, k-1}\left[b_{1} \otimes \cdots \otimes b_{k-1} b_{k}\right]-X_{\alpha-1, k-1}\left[b_{1} \otimes \cdots \otimes b_{k-1}\right] b_{k}\right) .
\end{aligned}
$$

As a consequence of the previous lemma, we get the following:

Corollary 2.8 For any $\alpha \neq 1,2 \leq k, \ell \in \mathbb{N}, \ell<k$, and $b_{i} \in \mathscr{A}$,

$$
\begin{aligned}
& X_{\alpha, k}\left[b_{1} \otimes \cdots \otimes b_{\ell-2} \otimes h \otimes h^{-1} a h \otimes h \otimes b_{\ell+2} \otimes \cdots \otimes b_{k}\right]-X_{\alpha, k}\left[b_{1} \otimes \cdots \otimes b_{\ell-2} \otimes h \otimes a \otimes h \otimes b_{\ell+2} \otimes \cdots \otimes b_{k}\right] \\
& \quad=\frac{1}{1-\alpha}\left(X_{\alpha-1, k-1}\left[b_{1} \otimes \cdots \otimes b_{\ell-2} \otimes a \otimes h \otimes b_{\ell+2} \otimes \cdots \otimes b_{k}\right]-X_{\alpha-1, k-1}\left[b_{1} \otimes \cdots \otimes b_{\ell-2} \otimes h \otimes h^{-1} a h \otimes b_{\ell+2} \cdots \otimes b_{k}\right]\right) .
\end{aligned}
$$

For instance, using also (2.17) and (2.18),

$$
\begin{aligned}
X_{\alpha, 3}\left[h \otimes h^{-1} a h \otimes h\right]-X_{\alpha, 3}[h \otimes a \otimes h] & =\frac{1}{1-\alpha}\left(X_{\alpha-1,2}[a \otimes h]-X_{\alpha-1,2}\left[h \otimes h^{-1} a h\right]\right) \\
& =\frac{1}{1-\alpha}\left(X_{\alpha-1,2}[a \otimes h]-h^{-1} X_{\alpha-1,2}[h \otimes a] h\right) .
\end{aligned}
$$

For a potential use of this corollary, see Remark 3.2.

\subsection{If $h$ commutes with the $b_{\ell}$ 's}

When $h$ commutes with the arguments acted upon by the operator $X_{\alpha, k}$, the action of the latter is quite simple:

Lemma 2.9 Let $\alpha \in \mathbb{R}, k \in \mathbb{N}$ and $b_{\ell} \in \mathscr{A}$ with $1 \leq \ell \leq k$. If $\left[h, b_{\ell}\right]=0$ for any $\ell$, then

$$
X_{\alpha, k}\left[b_{1} \otimes \cdots \otimes b_{k}\right]=\frac{1}{k !} h^{-\alpha} b_{1} \cdots b_{k} .
$$

This is for instance the case either when $h=\mathbb{1}$ or when $h$ and the $b_{\ell}$ 's are diagonal matrices.

PROOF We have, using the equality (2.5),

$$
X_{\alpha, k}\left[b_{1} \otimes \cdots \otimes b_{k}\right]=I_{\alpha, k}\left(r_{0}, \ldots, r_{k}\right) E_{0} b_{1} E_{1} \cdots b_{k} E_{k}=I_{\alpha, k}\left(r_{0}, \ldots, r_{0}\right) E_{0} b_{1} \cdots b_{k}=\frac{1}{k !} h^{-\alpha} b_{1} \cdots b_{k} .
$$

This shows that in this situation the operators $X_{\alpha, k}$ act as a polynomial in $h$ and the $b_{\ell}$ 's.

\subsection{Action of a derivation and finite differences}

It is immediate to extend all previous definitions and results to the algebra that we consider from now on, namely

$$
\mathscr{A}:=C^{\infty}\left(\mathscr{U}, M_{N}(\mathbb{C})\right)
$$


where $\mathscr{U}$ is a parameter space. Later on, when $P$ will play a role, $h$ will be either $u(x)$ with $\mathscr{U}=U$ (an open set in $M$ ) or $H(x, \xi)=g^{\mu v}(x) u(x) \xi_{\mu} \xi_{v}$ and $\mathscr{U}=U \times \mathbb{R}^{d}$. This extension is necessary because we have derivations in the play and consequently, the operators $X_{\alpha, k}$ now depend on $x \in M$. With the definitions

$$
\begin{aligned}
& f_{k, \ell}(s ; r):=e^{-\left(s_{\ell}-s_{\ell+1}\right) r}, \\
& \widetilde{I}_{k}\left(r_{0}, \ldots, r_{k}\right):=\int_{\Delta_{k}} \mathrm{~d} s f_{k, 0}\left(s ; r_{0}\right) \cdots f_{k, k}\left(s ; r_{k}\right),
\end{aligned}
$$

where $s \in \Delta_{k}, r \in \mathbb{R}_{+}^{*}$, we can rewrite the functions $I_{\alpha, k}$ as

$$
I_{\alpha, k}\left(r_{0}, \ldots, r_{k}\right)=\Gamma(\alpha)^{-1} \int_{\Delta_{k}} \mathrm{~d} s \int_{0}^{\infty} \mathrm{d} t t^{\alpha-1} e^{-t\left[\sum_{\ell=0}^{k}\left(s_{\ell}-s_{\ell+1}\right) r_{\ell}\right]}=\Gamma(\alpha)^{-1} \int_{0}^{\infty} \mathrm{d} t t^{\alpha-1} \widetilde{I}_{k}\left(t r_{0}, \ldots, t r_{k}\right)
$$

and the operator $f_{k}(\xi)$ defined in $(1.8)$ and restricted to $\mathscr{A}^{\otimes^{k}}$ (i.e. to arguments without derivatives), is associated for $h=H(x, \xi)$ to the spectral function $\widetilde{I}_{k}$ :

$$
f_{k}(\xi)=\pi_{\widetilde{I}_{k}} .
$$

Let $\partial$ be an arbitrary derivation of the algebra $\mathscr{A}$, namely a linear combination of a derivative of a $\mathscr{A}$-valued function along a parameter $y \in \mathscr{U}$ and a commutator with an element of $\mathscr{A}$. Then

$$
\partial e^{-t h}=-\int_{0}^{t} \mathrm{~d} s e^{(s-t) h}(\partial h) e^{-s h} .
$$

Recall that a proof for $\partial=\partial_{y}$ is based on the following relation: If $E_{\epsilon}(s):=e^{(s t-t) h(y+\epsilon)} e^{-s t h(y)}$ for $s \in[0,1]$ and $\epsilon \in \mathbb{R}$, then

$$
e^{-t h(y+\epsilon)}-e^{-t h(y)}=-\int_{0}^{1} \mathrm{~d} s E_{\epsilon}^{\prime}(s)=-\int_{0}^{1} t \mathrm{~d} s e^{(s t-t) h(y+\epsilon)}(h(y+\epsilon)-h(y)) e^{-s t h(y)},
$$

see also [4]. For an inner derivation like $\partial=[\cdot, a]$ where $a \in \mathscr{A}$, a similar argument applies beginning with $E(s):=$ $e^{(s t-t) h} a e^{-s t h}$.

By functional calculus on $h=r_{0} E_{0}$, we deduce from (2.33)

$$
\partial e^{-t h}=-\int_{0}^{t} \mathrm{~d} s e^{(s-t) r_{0}} e^{-s r_{1}} E_{0}(\partial h) E_{1}=\frac{e^{-t r_{1}}-e^{-t r_{0}}}{r_{1}-r_{0}} E_{0}(\partial h) E_{1},
$$

(still an implicit summation over repeated indices) where from now on we use the symbolic notation $\frac{f\left(r_{1}\right)-f\left(r_{0}\right)}{r_{1}-r_{0}}$ of finite differences instead of $f^{\prime}\left(r_{0}\right)$ when $r_{0}=r_{1}$.

Remark that the peculiar case $\partial_{\xi_{\mu}} e^{-t H(x, \xi)}=-t g^{\mu \nu} \xi_{\nu} u(x) e^{-t H(x, \xi)}$ is compatible with (2.34).

The relation (2.34) can be extended in the following way. Let $g$ be a Laplace transform of a Borel signed $\mathbb{R}$-valued measure $\phi$ on $\mathbb{R}_{+}$, i.e. $g: r \in \mathbb{R}_{+}^{*} \rightarrow \int_{0}^{\infty} \mathrm{d} \phi(t) e^{-t r} \in \mathbb{R}$. We consider the derivability of $g$ at the point $\left.r_{0} \in\right] r_{m}, r_{M}[$ : since for any $r \in] r_{m}, r_{M}\left[\right.$ and $0<\epsilon<r_{m},\left|\partial_{r}\left(t e^{-t r}\right)\right|<\epsilon^{-1} e^{t \epsilon} e^{-t r_{m}}$ and $\int_{0}^{\infty} \mathrm{d} \phi(t) e^{-t\left(r_{m}-\epsilon\right)}=g\left(r_{m}-\epsilon\right)<\infty$, we may use the differentiation lemma for parameter dependent integrals to commute $\partial_{r}$ and the integral (recall also that by Bernstein's theorem, $g$ is completely monotonic, see [17, Theorem 1.4]). This commutation property can be first extended when $g$ is a Laplace transform of a signed $\mathbb{C}$-valued measure $\phi$ on $\mathbb{R}_{+}$and then extended again to $f=g \circ r$ for any smooth function $r: y \in \mathscr{U} \rightarrow \mathbb{R}_{+}^{*}$ : consider again the derivability of $f$ in an open ball $B$ around $y_{0}$ such that $\left.r\left(y_{0}\right) \in r(B) \subset\right] r_{m}, r_{M}$ [ where $r_{m}=\inf _{y \in \bar{B}} r(y)$ and $r_{M}=\sup _{y \in \bar{B}} r(y)$; as before we get $\left|\partial_{y} e^{-t r(y)}\right|=\left|r^{\prime}(y)\right| t e^{-t r(y)}<\sup _{y \in \bar{B}}\left|r^{\prime}(y)\right| \epsilon^{-1} e^{-t\left(r_{m}-\epsilon\right)}$ so that we can commute $\partial_{y}$ with the integral. In particular, for such $f$,

$$
\partial[f(h)]=\int_{0}^{\infty} \mathrm{d} \phi(t) \partial\left(e^{-t r_{0} E_{0}}\right)=\frac{1}{r_{1}-r_{0}}\left[\int_{0}^{\infty} \mathrm{d} \phi(t)\left(e^{-t r_{1}}-e^{-t r_{0}}\right)\right] E_{0}(\partial h) E_{1}=\frac{f\left(r_{1}\right)-f\left(r_{0}\right)}{r_{1}-r_{0}} E_{0}(\partial h) E_{1} .
$$

It is then natural to define the set of functions

$$
\mathscr{C}:=\left\{f \in C^{\infty}\left(\mathbb{R}_{+}^{*}, \mathbb{C}\right) \mid \partial[f(h)]=\frac{f\left(r_{1}\right)-f\left(r_{0}\right)}{r_{1}-r_{0}} E_{0}(\partial h) E_{1}\right\}
$$


which is large because $\mathscr{C}$ contains all functions of type $g \circ r$ with $g, r$ defined as above.

Since $r^{-\alpha}=\frac{1}{\Gamma(\alpha)} \int_{0}^{\infty} \mathrm{d} t t^{\alpha-1} e^{-t r}$ for $\alpha>0$, the definition (2.4) shows in particular that $I_{\alpha, 0} \in \mathscr{C}$ if $\alpha>0$ and since $I_{0,0}=1$,

$$
I_{\alpha, 0} \in \mathscr{C}, \quad \forall \alpha \geq 0 .
$$

With the help of (2.31), the functions

$$
I_{\alpha, k}\left(r_{0}, \ldots, r_{k}\right)=\Gamma(\alpha)^{-1} \int_{\Delta_{k}} \mathrm{~d} s \int_{0}^{\infty} \mathrm{d} t t^{\alpha-1} f_{k, 0}\left(s ; t r_{0}\right) \cdots f_{k, k}\left(s ; t r_{k}\right)
$$

for $\alpha>0$ are nothing else but integrals of products of elements in $\mathscr{C}$.

Then, let us introduce the following generalization of the finite difference appearing in the RHS of (2.35) to functions of several variables: for $f \in C^{\infty}\left(\left(\mathbb{R}_{+}^{*}\right)^{k+1}, \mathbb{C}\right)$ and $k, \ell \in \mathbb{N}$ with $0 \leq \ell \leq k$, define the "partial" finite differences

$$
\left(\Delta^{(\ell)} f\right)\left(r_{0}, \ldots, r_{k+1}\right):= \begin{cases}\frac{1}{r_{\ell+1}-r_{\ell}}\left(f\left(r_{0}, \ldots, \widehat{r_{\ell}}, \ldots, r_{k+1}\right)-f\left(r_{0}, \ldots, \widehat{r_{\ell+1}}, \ldots, r_{k+1}\right)\right), & \text { when } r_{\ell} \neq r_{\ell+1}, \\ \partial_{r_{\ell}} f\left(r_{0}, \cdots, r_{\ell}, r_{\ell}, \cdots, r_{k}\right), & \text { when } r_{\ell}=r_{\ell+1} .\end{cases}
$$

We can generalize (2.35) to operators $\pi_{f}$ by defining:

$$
\Delta_{\partial}^{(\ell)} \pi_{f}:=i_{\partial h}^{(\ell+1)} \pi_{\Delta^{(\ell) f}},
$$

or more explicitly, $\Delta_{\partial}^{(\ell)} \pi_{f}=\left(\Delta^{(\ell)} f\right)\left(r_{0}, \ldots, r_{k+1}\right) \mathbf{m} \circ\left(E_{0}^{R} \otimes \cdots \otimes\left[E_{\ell}(\partial h) E_{\ell+1}\right]^{R} \otimes \cdots \otimes E_{k+1}^{R}\right)$. The next proposition shows that the families of $I_{\alpha, k}$ and $X_{\alpha, k}$ are indeed invariant respectively by $\Delta^{(\ell)}$ and $\Delta_{\partial}^{(\ell)}$ modulo dilations and insertion of $\partial h$ :

Proposition 2.10 For any $\alpha \neq 1$, any $k, \ell \in \mathbb{N}^{*}$ with $0 \leq \ell \leq k$, we have

$$
\begin{aligned}
& \Delta^{(\ell)} I_{\alpha, k}=-\alpha I_{\alpha+1, k+1}, \\
& \Delta_{\partial}^{(\ell)} X_{\alpha, k}=-\alpha i_{\partial h}^{(\ell+1)} X_{\alpha+1, k+1}, \\
& \Delta^{(\ell)} \widetilde{I}_{k}=-\widetilde{I}_{k+1}, \\
& \Delta_{\partial}^{(\ell)} f_{k}(\xi)=-i_{\partial h}^{(\ell+1)} f_{k+1}(\xi) \text { with } h=H(x, \xi),
\end{aligned}
$$

PROoF The first relation follows from (2.8) applied to $I_{\alpha+1, k+1}$ with $\ell+1$ instead of $\ell$ and the second one from the definition (2.15). The third one can be shown using a straightforward adaptation of the proof of Lemma 2.2 ii) beginning with $\widetilde{I}_{k+1}\left(r_{0}, \ldots, r_{k+1}\right)=\int_{\Delta_{k+1}} \mathrm{~d} s e^{-\sum_{\ell=0}^{k+1} s_{\ell}\left(r_{\ell}-r_{\ell-1}\right)}$. The last relation is a consequence of (2.32) and (2.43).

\subsection{Propagation of derivations}

Let $\partial$ be a derivation acting on elements in $\mathscr{A}$, for instance along a parameter in $\mathscr{U}$. Suppose that we have a representation of the algebra $\mathscr{A}$ on a vector space $\mathscr{H}$ on which $\partial$ is also defined (with the same notations) in such a way that the Leibniz rule holds: $\partial(a v)=(\partial a) v+a(\partial v)$ for any $a \in \mathscr{A}$ and $v \in \mathscr{H}$.

Proposition 2.11 Assume that the function $f \in C^{\infty}\left(\left(\mathbb{R}_{+}^{*}\right)^{k+1}, \mathbb{C}\right)$ is either $f\left(r_{0}, \ldots, r_{k}\right)=f_{0}\left(r_{0}\right) \cdots f_{k}\left(r_{k}\right)$ with $f_{\ell} \in \mathscr{C}$, or $f=\widetilde{I}_{k}$, or $f=I_{\alpha, k}$ with $\alpha \geq 0$. Then, for any $\ell$ such that $1 \leq \ell \leq k$ and any $b_{\ell}$ which is a $\mathscr{A}$-valued differential operator in $\partial$, the following propagation rule holds true:

$$
\begin{array}{r}
\pi_{f}\left[b_{1} \otimes \cdots \otimes b_{\ell} \partial \otimes \cdots \otimes b_{k}\right] v=\sum_{i=\ell+1}^{k} \pi_{f}\left[b_{1} \otimes \cdots \otimes b_{i-1} \otimes\left(\partial b_{i}\right) \otimes \cdots \otimes b_{k}\right] v+\sum_{i=\ell}^{k}\left(\Delta_{\partial}^{(i)} \pi_{f}\right)\left[b_{1} \otimes \cdots \otimes b_{k}\right] v \\
+\pi_{f}\left[b_{1} \otimes \cdots \otimes b_{\ell} \otimes \cdots \otimes b_{k}\right](\partial v) .
\end{array}
$$

Before looking at a proof, remark first that, using (2.42) for $X_{\alpha, k}=\pi_{I_{\alpha, k}}$, see (2.15), (resp. $f_{k}(\xi)=\pi_{\widetilde{I}_{\underline{k}}}$, see (2.32)), the RHS of (2.45) is written only in terms of the operators $X_{\alpha, k}$ and $X_{\alpha+1, k+1}$ (resp. $f_{k}(\xi)$ and $f_{k+1}(\xi)$, using (2.44)). This is a key point in the method exhibited in Section 4. 
Proof First case: Since $\pi_{f}=\mathbf{m} \circ\left(\left(f_{0}\left(r_{0}\right) E_{0}\right)^{R} \otimes \cdots \otimes\left(f_{k}\left(r_{k}\right) E_{k}\right)^{R}\right)$, one gets

$$
\pi_{f}\left[b_{1} \otimes \cdots \otimes b_{\ell} \partial \otimes \cdots \otimes b_{k}\right] v=f_{0}\left(r_{0}\right) E_{0} b_{1} f_{1}\left(r_{1}\right) E_{1} \cdots b_{\ell} \partial\left(f_{\ell}\left(r_{\ell}\right) E_{\ell} b_{\ell+1} \cdots b_{k} f_{k}\left(r_{k}\right) E_{k} v\right) .
$$

Thus, in the parenthesis, $\partial$ acts either on the $b_{i}$ 's or on the $f_{i}\left(r_{i}\right) E_{i}$ 's or on $v$. When it acts on the $b_{i}$ 's, this reproduces the first sum in (2.45) while when it acts on $v$, it gives the last term.

For the action on the $f_{i}\left(r_{i}\right) E_{i}=f_{i}(h)$, we can apply (2.35) and get the total contribution

$$
\begin{aligned}
& \sum_{i=\ell}^{k} f_{0}\left(r_{0}\right) E_{0} b_{1} \cdots b_{i} \partial\left(f_{i}\left(r_{i}\right) E_{i}\right) b_{i+1} \cdots b_{k} f_{k}\left(r_{k}\right) E_{k} v \\
& \quad=\sum_{i=\ell}^{k} f_{0}\left(r_{0}\right) E_{0} b_{1} \cdots b_{i} \frac{f_{i}\left(r_{i}\right)-f_{i}\left(r_{j}\right)}{r_{i}-r_{j}} E_{i}(\partial h) E_{j} b_{i+1} \cdots b_{k} f_{k}\left(r_{k}\right) E_{k} v \\
& \quad=\sum_{i=\ell}^{k} f_{0}\left(r_{0}\right) \cdots \frac{f_{i}\left(r_{i}\right)-f_{i}\left(r_{j}\right)}{r_{i}-r_{j}} \cdots f_{k}\left(r_{k}\right) E_{0} b_{1} \cdots b_{i} E_{i}(\partial h) E_{j} b_{i+1} \cdots b_{k} E_{k} v
\end{aligned}
$$

Swapping $r_{j}$ to $r_{i+1}$ and $r_{j}$ to $r_{j+1}$ for $j>i$, each spectral function in the last sum is

$$
\begin{aligned}
f_{0}\left(r_{0}\right) \cdots \frac{f_{i}\left(r_{i}\right)-f_{i}\left(r_{i+1}\right)}{r_{i}-r_{i+1}} \cdots f_{k}\left(r_{k+1}\right)= & \frac{1}{r_{i}-r_{i+1}}\left[f_{0}\left(r_{0}\right) \cdots f_{i-1}\left(r_{i-1}\right) f_{i}\left(r_{i}\right) f_{i+1}\left(r_{i+2}\right) \cdots f_{k}\left(r_{k+1}\right)\right. \\
& \left.\quad-f_{0}\left(r_{0}\right) \cdots f_{i-1}\left(r_{i-1}\right) f_{i}\left(r_{i+1}\right) f_{i+1}\left(r_{i+2}\right) \cdots f_{k}\left(r_{k+1}\right)\right] \\
= & \frac{1}{r_{i+1}-r_{i}}\left[f\left(r_{0}, \ldots, \widehat{r_{i}}, \ldots, r_{k+1}\right)-f\left(r_{0}, \ldots, \widehat{r_{i+1}}, \ldots, r_{k+1}\right)\right]=\Delta^{(i)} f\left(r_{0}, \ldots, r_{k+1}\right)
\end{aligned}
$$

which contributes to the second term of the RHS of (2.45).

Case $f=\widetilde{I}_{k}$ : From (2.29), (2.30) and using (2.13), we can apply the previous argument under the integration over $\Delta_{k}$. The only point to take care of, is the commutation of $\Delta^{(i)}$ with the integral in the second term of the RHS of (2.45). Since $\Delta_{k}$ is compact and the integrand is smooth in $s$ and $r_{\ell}$, this commutation occurs even at coincidence points (where partial derivatives arise, see (2.39)).

Case $f=I_{\alpha, k}$ : For $\alpha=0$, the result is direct and the second term of (2.45) vanishes since $I_{0, k}$ is constant.

Assume now $\alpha>0$. From (2.38), once again the same argument can be applied under the integral and we only need to prove the commutation with integral along $t \in[0, \infty[$. With

$$
f\left(t ; s ; r_{0}, \ldots, r_{k}\right):=e^{-t \sum_{\ell=0}^{k}\left(s_{\ell}-s_{\ell+1}\right) r_{\ell}}, \quad g\left(r_{0}, \ldots, r_{k}\right):=\int_{0}^{\infty} d t t^{\alpha-1} f\left(t ; s ; r_{0}, \ldots, r_{k}\right)=\Gamma(\alpha)\left[\sum_{\ell=0}^{k} s_{\ell}\left(r_{\ell}-r_{\ell-1}\right)\right]^{-\alpha},
$$

we have to show that

$$
\left(\Delta^{(i)} \int_{0}^{\infty} d t t^{\alpha-1} f(t ; s ; \cdot)\right)\left(r_{0}, \ldots, r_{k+1}\right)=\frac{1}{r_{i+1}-r_{i}}\left(g\left(r_{0}, \ldots, \widehat{r}_{i}, \ldots, r_{k}\right)-g\left(r_{0}, \ldots, \widehat{r_{i+1}}, \ldots, r_{k}\right)\right)
$$

coincides with

$$
\int_{0}^{\infty} \mathrm{d} t t^{\alpha-1}\left(\Delta^{(i)} f(t ; s ; \cdot)\right)\left(r_{0}, \ldots, r_{k+1}\right)=\frac{1}{r_{i+1}-r_{i}} \int_{0}^{\infty} \mathrm{d} t t^{\alpha-1}\left(e^{-t \sum_{\ell=0 ; \ell \neq i}^{k} s_{\ell}\left(r_{\ell}-r_{\ell-1}\right)}-e^{-t \sum_{\ell=0 ; \ell \neq i+1}^{k} s_{\ell}\left(r_{\ell}-r_{\ell-1}\right)}\right) .
$$

By linearity, this is true for $r_{i} \neq r_{i+1}$ but at coincidence points we still have to show that the $t$-integral commutes with $\partial_{r_{i}}$. For $\left.r_{i} \in\right] r_{\min }=\min r_{j}, r_{\max }=\max r_{j}$ [ we have, using $t\left(s_{i}-s_{i+1}\right) e^{-t\left(s_{i}-s_{i+1}\right) r_{i}} \leq \epsilon^{-1} e^{-t\left(s_{i}-s_{i+1}\right)\left(r_{\min }-\epsilon\right)}$ when $0<\epsilon<r_{\min }$,

$$
t^{\alpha-1}\left|\left(\partial_{r_{i}} f\right)\left(t ; s ; r_{0}, \ldots, r_{k}\right)\right|=\left(s_{i}-s_{i+1}\right) t^{\alpha} f\left(t ; s ; r_{0}, \ldots, r_{k}\right) \leq \epsilon^{-1} t^{\alpha-1} f\left(t ; s ; r_{0}, \ldots, r_{i-1}, r_{\min }-\epsilon, r_{i+1}, \ldots, r_{k}\right)
$$

and the RHS is $t$-integrable uniformly along $r_{i}$ which secures the commutation of the integral with $\partial_{r_{i}}$.

\section{Total covariant derivative and normal coordinates}

In this section, we come back to the differential operator $P$ defined in (1.1) but we do not use Section 2. Firstly, we rewrite $P$ in terms of a total covariant derivative, and since we know that the coefficients $\mathscr{R}_{r}$ are invariant under a change of coordinates, we secondly gather the computation of several derivatives within a normal coordinate system. 


\subsection{Total covariant derivative}

We need the total covariant derivative $\widehat{\nabla}$, which combines the (gauge) connection $\nabla$ on $V$ with the Levi-Civita covariant derivative ${ }^{g} \nabla$ induced by the metric $g$. To avoid a definition of $\widehat{\nabla}$ on the tensor products of $V, T M$ and $T^{*} M$ via heavy notations, and since we only need the action on $\operatorname{End}(V)$-valued tensors, it is sufficient to remark that $\widehat{\nabla}$ satisfies

$$
\begin{aligned}
& \widehat{\nabla}_{\mu} u=\nabla_{\mu} u=\partial_{\mu} u+\left[A_{\mu}, u\right], \\
& \widehat{\nabla}_{\mu} a^{v}=\nabla_{\mu} a^{v}+\Gamma_{\mu \rho}^{v} a^{\rho}, \quad \widehat{\nabla}_{\mu} b_{v}=\nabla_{\mu} b_{\nu}-\Gamma_{\mu \nu}^{\rho} b_{\rho}, \\
& \widehat{\nabla}_{\mu} g^{\alpha \beta}=0, \quad \widehat{\nabla}_{\mu} g_{\alpha \beta}=0,
\end{aligned}
$$

for any End(V)-valued (0,0)-tensor $u,(1,0)$-tensor $a=a^{v} \partial_{v}$, and (0,1)-tensor $b=b_{v} \mathrm{~d} x^{v}$. Here $A_{\mu}$ is the (local) gauge potential associated to $\nabla$. General formulas for $\operatorname{End}(V)$-valued $(r, s)$-tensors are easily obtained from these conventions. As usual, we use the short notation $\widehat{\nabla}_{\mu} a^{v}=\left(\widehat{\nabla}_{\mu} a\right)^{v}$ and $\widehat{\nabla}_{\mu} b_{v}=\left(\widehat{\nabla}_{\mu} b\right)_{v}$.

We first recall few formulae of Riemannian geometry:

$$
\begin{aligned}
& |g|^{-1 / 2} \partial_{\mu}|g|^{1 / 2}=\frac{1}{2} \partial_{\mu} \ln |g|, \\
& \Gamma_{\mu \nu}^{\mu}=-\frac{1}{2} g_{\alpha \beta}\left(\partial_{\nu} g^{\alpha \beta}\right), \\
& \Gamma^{\rho}=g^{\mu \nu} \Gamma_{\mu \nu}^{\rho}=\frac{1}{2} g^{\rho \sigma} g_{\alpha \beta}\left(\partial_{\sigma} g^{\alpha \beta}\right)-\partial_{\sigma} g^{\rho \sigma}=-g^{\rho \sigma} \frac{1}{2} \partial_{\sigma} \ln |g|-\partial_{\sigma} g^{\rho \sigma} .
\end{aligned}
$$

The curvature of the Levi-Civita connection ${ }^{g} \nabla$ is

$$
R(X, Y):={ }^{g} \nabla_{X}{ }^{g} \nabla_{Y}-{ }^{g} \nabla_{Y}{ }^{g} \nabla_{X}-{ }^{g} \nabla_{[X, Y]},
$$

([11, p. 23$])$ and this expression is an endomorphism of the tangent bundle $T M$.

The Riemann tensors

$$
R_{\mu \nu \rho}{ }^{\sigma} \partial_{\sigma}:=R\left(\partial_{\mu}, \partial_{v}\right) \partial_{\rho}, \quad R_{\mu \nu \rho \sigma}:=g_{\sigma \alpha} R_{\mu \nu \rho}^{\alpha}=g\left(R\left(\partial_{\mu}, \partial_{v}\right) \partial_{\rho}, \partial_{\sigma}\right),
$$

satisfy $R_{\mu \nu \rho}^{\sigma}=\partial_{\mu} \Gamma_{\nu \rho}^{\sigma}-\partial_{\nu} \Gamma_{\mu \rho}^{\sigma}+\Gamma_{\mu \gamma}^{\sigma} \Gamma_{\nu \rho}^{\gamma}-\Gamma_{\nu \gamma}^{\sigma} \Gamma_{\mu \rho}^{\gamma}$, with $\Gamma_{\mu \nu}^{\rho} \partial_{\rho}:={ }^{g} \nabla_{\partial_{\mu}} \partial_{\nu}$.

The Ricci tensor $\operatorname{Ric}_{\mu \nu}$ and the scalar curvature $\mathfrak{R}$ are

$$
\operatorname{Ric}_{\mu \nu}:=g^{\alpha \beta} R_{\mu \alpha \beta \nu}=\operatorname{Ric}_{v \mu}, \quad \Re:=g^{\mu \nu} \operatorname{Ric}_{\mu \nu} .
$$

The Riemann tensor yields to some $\mathbb{Z}_{2}$-symmetries and to the first Bianchi identity:

$$
R_{\mu \nu \rho \sigma}=R_{\rho \sigma \mu \nu}=-R_{\nu \mu \rho \sigma}=-R_{\mu \nu \sigma \rho}, \quad R_{\mu \nu \rho \sigma}+R_{\mu \rho \sigma \nu}+R_{\mu \sigma \nu \rho}=0 .
$$

This also yields to some $\mathbb{Z}_{2}$-symmetries, to the derivative of the first Bianchi identity and the second Bianchi identity:

$$
\begin{aligned}
& \left({ }^{g} \nabla_{\tau} R\right)_{\mu \nu \rho \sigma}=-\left({ }^{g} \nabla_{\tau} R\right)_{\nu \mu \rho \sigma}=-\left({ }^{g} \nabla_{\tau} R\right)_{\mu \nu \sigma \rho}=\left({ }^{g} \nabla_{\tau} R\right)_{\rho \sigma \mu \nu}, \\
& \left({ }^{g} \nabla_{\tau} R\right)_{\mu \nu \rho \sigma}+\left({ }^{g} \nabla_{\tau} R\right)_{\nu \rho \mu \sigma}+\left({ }^{g} \nabla_{\tau} R\right)_{\rho \mu \nu \sigma}=0, \\
& \left({ }^{g} \nabla_{\tau} R\right)_{\mu \nu \rho \sigma}+\left({ }^{g} \nabla_{\rho} R\right)_{\mu \nu \sigma \tau}+\left({ }^{g} \nabla_{\sigma} R\right)_{\mu \nu \tau \rho}=0 .
\end{aligned}
$$

In (3.2), after a contraction over $\mu$ and $\rho$ first and then over $v$ and $\sigma$, and using the fact that ${ }^{g} \nabla_{\tau} g^{\mu \rho}=0$, one obtains $-\left({ }^{g} \nabla_{\tau} \text { Ric }\right)_{\nu \sigma}+\left({ }^{g} \nabla_{\rho} R\right)^{\rho}{ }_{\nu \sigma \tau}+\left({ }^{g} \nabla_{\sigma} \text { Ric }\right)_{v \tau}=0$ and $-\left({ }^{g} \nabla_{\tau} \mathfrak{R}\right)+\left({ }^{g} \nabla_{\rho} \text { Ric }\right)^{\rho}{ }_{\tau}+\left({ }^{g} \nabla_{\nu} \text { Ric }\right)_{\tau}=0$, so that

$$
g^{\mu v}\left({ }^{g} \nabla_{\mu} \text { Ric }\right)_{v \rho}=\frac{1}{2}\left({ }^{g} \nabla_{\rho} \Re\right)
$$

If we define

$$
|R|^{2}:=R_{\mu_{1} \mu_{2} \mu_{3} \mu_{4}} R^{\mu_{1} \mu_{2} \mu_{3} \mu_{4}}
$$

then, by the first Bianchi identity, $R_{\mu_{1} \mu_{2} \mu_{3} \mu_{4}} R^{\mu_{1} \mu_{3} \mu_{2} \mu_{4}}=-R_{\mu_{1} \mu_{2} \mu_{3} \mu_{4}} R^{\mu_{1} \mu_{2} \mu_{4} \mu_{3}}-R_{\mu_{1} \mu_{2} \mu_{3} \mu_{4}} R^{\mu_{1} \mu_{4} \mu_{3} \mu_{2}}=|R|^{2}-R_{v_{1} v_{2} v_{3} v_{4}} R^{v_{1} v_{3} v_{2} v_{4}}$ with a relabeling for the second equality, so that

$$
\frac{1}{2}|R|^{2}=R_{\mu_{1} \mu_{2} \mu_{3} \mu_{4}} R^{\mu_{1} \mu_{3} \mu_{2} \mu_{4}}
$$


In the same vein, let us store

$$
|\operatorname{Ric}|^{2}:=\operatorname{Ric}_{\mu_{1} \mu_{2}} \operatorname{Ric}^{\mu_{1} \mu_{2}}=\operatorname{Ric}_{\mu_{1} \mu_{2}} \operatorname{Ric}^{\mu_{2} \mu_{1}} .
$$

Given the field strength

$$
F_{\mu \nu}:=\partial_{\mu} A_{\nu}-\partial_{\nu} A_{\mu}+\left[A_{\mu}, A_{\nu}\right]
$$

one has for any section $u$ of $\operatorname{End}(V)$ and similarly, for any tensors $a=a^{v} \partial_{\mu}$ and $b=b_{\nu} \mathrm{d} x^{v}$,

$$
\left[\nabla_{\mu}, \nabla_{\nu}\right] u=\left[F_{\mu \nu}, u\right], \quad\left[{ }^{g} \nabla_{\mu},{ }^{g} \nabla_{\nu}\right] a^{\rho}=R_{\mu \nu \sigma}{ }^{\rho} a^{\sigma}, \quad\left[{ }^{g} \nabla_{\mu},{ }^{g} \nabla_{\nu}\right] b_{\rho}=-R_{\mu \nu \rho}{ }^{\sigma} b_{\sigma} .
$$

Combining these expressions, for any End(V)-valued tensors $a=a^{\mu} \partial_{\mu}$ and $b=b_{\nu} \mathrm{d} x^{\nu}$, one obtains

$$
\left[\widehat{\nabla}_{\mu}, \widehat{\nabla}_{\nu}\right] u=\left[F_{\mu \nu}, u\right], \quad\left[\widehat{\nabla}_{\mu}, \widehat{\nabla}_{\nu}\right] a^{\sigma}=\left[F_{\mu \nu}, a^{\rho}\right]+R_{\mu \nu \rho}{ }^{\sigma} a^{\rho}, \quad\left[\widehat{\nabla}_{\mu}, \widehat{\nabla}_{\nu}\right] b_{\rho}=\left[F_{\mu \nu}, b_{\rho}\right]-R_{\mu \nu \rho}{ }^{\sigma} b_{\sigma},
$$

and for any $s \in \Gamma(V)$

$$
\left[\widehat{\nabla}_{\mu}, \widehat{\nabla}_{\nu}\right] s=F_{\mu \nu} s .
$$

Moreover,

$$
\begin{aligned}
& g^{\mu \nu} \widehat{\nabla}_{\mu} \widehat{\nabla}_{\nu} u=g^{\mu \nu}\left(\nabla_{\mu} \nabla_{v} u-\Gamma_{\mu \nu}^{\rho} \nabla_{\rho} u\right)=g^{\mu \nu} \nabla_{\mu} \nabla_{v} u-\Gamma^{\mu} \nabla_{\mu} u \\
& \widehat{\nabla}_{\mu} p^{\mu}=\nabla_{\mu} p^{\mu}+\Gamma_{\mu \rho}^{\mu} p^{\rho}=\nabla_{\mu} p^{\mu}-\frac{1}{2} g_{\alpha \beta}\left(\partial_{\mu} g^{\alpha \beta}\right) p^{\mu} .
\end{aligned}
$$

Let us now come back to the operator $P$. Applying the definition (1.5), we can rewrite $P$ in a (fully) covariant way as

$$
P=-\left(g^{\mu \nu} \widehat{\nabla}_{\mu} u \widehat{\nabla}_{v}+p^{v} \widehat{\nabla}_{v}+q\right)=-\left(g^{\mu v} u \widehat{\nabla}_{\mu} \widehat{\nabla}_{v}+N^{v} \widehat{\nabla}_{v}+q\right),
$$

and the following result is a generalization of [12, Lemma 1.2.1]:

Lemma 3.1 Given $P$ as in (3.8), there exist a connection $\widehat{\nabla}^{\prime}$ and a section $q^{\prime}$ of End( $\left.V\right)$ such that

$$
P=-\left(g^{\mu v} u \widehat{\nabla}_{\mu}^{\prime} \widehat{\nabla}_{v}^{\prime}+q^{\prime}\right)
$$

given by $\widehat{\nabla}_{\mu}^{\prime}:=\widehat{\nabla}_{\mu}+\frac{1}{2} u^{-1} N_{\mu}$ and $q^{\prime}:=q-\frac{1}{2} g^{\mu v} u \widehat{\nabla}_{\mu}\left(u^{-1} N_{v}\right)-\frac{1}{4} N^{v} u^{-1} N_{v}$.

ProOF This follows from a direct computation (omitting the section applied upon):

$$
\begin{aligned}
g^{\mu \nu} u \widehat{\nabla}_{\mu}^{\prime} \widehat{\nabla}_{v}^{\prime}+q^{\prime} & =g^{\mu v} u\left(\widehat{\nabla}_{\mu}+\frac{1}{2} u^{-1} N_{\mu}\right)\left(\widehat{\nabla}_{v}+\frac{1}{2} u^{-1} N_{v}\right)+q-\frac{1}{2} g^{\mu v} u \widehat{\nabla}_{\mu}\left(u^{-1} N_{v}\right)-\frac{1}{4} N^{v} u^{-1} N_{v} \\
& =+g^{\mu \nu}\left[u \widehat{\nabla}_{\mu} \widehat{\nabla}_{v}+\frac{1}{2} u \widehat{\nabla}_{\mu}\left(u^{-1} N_{v}\right)+\frac{1}{2} N_{v} \widehat{\nabla}_{\mu}+\frac{1}{2} N_{\mu} \widehat{\nabla}_{v}+\frac{1}{4} N_{\mu} u^{-1} N_{v}-\frac{1}{2} u \widehat{\nabla}_{\mu}\left(u^{-1} N_{v}\right)\right]+q-\frac{1}{4} N^{v} u^{-1} N_{v} \\
& =g^{\mu \nu}\left[u \widehat{\nabla}_{\mu} \widehat{\nabla}_{v}+N_{\mu} \widehat{\nabla}_{v}\right]+q=-P .
\end{aligned}
$$

Remark 3.2 Compared with (3.8), this rewriting of $P$ in (3.9) greatly simplifies the computations of $\mathscr{R}_{r}$ because it means that we may assume $N^{v}=0$ for all $v$. This is the traditional way to present the results on the heat kernel coefficients, see for instance $[11,12]$. In particular, $N^{v}=0$ for all $v$ is equivalent to $L^{\mu}=-\Gamma^{\mu} u$ in (1.6).

If one insists on keeping $N^{\mu} \neq 0$, Corollary 2.8 can be useful because $\widehat{\nabla}_{\mu}^{\prime} u=\widehat{\nabla}_{\mu} u+\frac{1}{2} u^{-1} N_{\mu} u$.

\subsection{Covariant derivatives and normal coordinates}

In this subsection, some results on the iterated covariant derivatives of $u, p^{\mu}, \ldots$ are shown and they will be used for the computation of $\mathscr{R}_{2}$. The code generates their generalizations to higher orders in derivatives for the computation of $\mathscr{R}_{4}$. In the following, for any $\ell \in \mathbb{N}$ and indices $v_{1}, \ldots, v_{\ell}$, we use the compact notation

$$
\nabla_{v_{1} \ldots v_{\ell}}^{\ell}:=\nabla_{v_{1}} \cdots \nabla_{v_{\ell}} \text { and the same for } \widehat{\nabla} \text { and } \partial \text {. }
$$

Comparing the action of $\widehat{\nabla}$ and $\nabla$ on $u$, we get

$$
\widehat{\nabla}_{v_{1}} u=\nabla_{v_{1}} u, \quad \widehat{\nabla}_{v_{1} v_{2}}^{2} u=\nabla_{v_{1} v_{2}}^{2} u-\Gamma_{v_{1} v_{2}}^{\sigma_{1}}\left(\widehat{\nabla}_{\sigma_{1}} u\right) \text {. }
$$


We deduce from (3.10) that if $u$ is parallel for $\nabla$ (i.e. $\nabla_{\mu} u=0$ ), then $u$ is also parallel for $\widehat{\nabla}$. Similarly,

$$
\widehat{\nabla}_{v_{1}} p^{\mu_{1}}=\nabla_{v_{1}} p^{\mu_{1}}+\Gamma_{v_{1} \sigma_{1}}^{\mu_{1}} p^{\sigma_{1}} .
$$

For $L^{\mu}=N^{\mu}-\Gamma^{\mu} u=p^{\mu}+g^{\mu \nu}\left(\nabla_{v} u\right)-\Gamma^{\mu} u$,

$$
\begin{aligned}
& \nabla_{v_{1}} L^{\mu_{1}}=\nabla_{v_{1}} N^{\mu_{1}}-\Gamma^{\mu_{1}}\left(\nabla_{v_{1}} u\right)-\left(\partial_{v_{1}} \Gamma^{\mu_{1}}\right) u, \\
& \nabla_{v_{1}} N^{\mu_{1}}=\nabla_{v_{1}} p^{\mu_{1}}+g^{\mu_{1} \sigma_{1}}\left(\nabla_{v_{1} \sigma_{1}}^{2} u\right)+\left(\partial_{v_{1}} g^{\mu_{1} \sigma_{1}}\right)\left(\nabla_{\sigma_{1}} u\right) .
\end{aligned}
$$

To compute the RHS of these expressions, we also need to know the derivatives of $\Gamma_{\alpha \beta}^{\gamma}$ and $\Gamma^{\gamma}$ :

$$
\begin{aligned}
\Gamma_{\alpha \beta}^{\gamma} & =\frac{1}{2} g^{\gamma \sigma_{1}}\left(\partial_{\alpha} g_{\sigma_{1} \beta}+\partial_{\beta} g_{\sigma_{1} \alpha}-\partial_{\sigma_{1}} g_{\alpha \beta}\right), \\
\partial_{\nu_{1}} \Gamma_{\alpha \beta}^{\gamma} & =\frac{1}{2}\left[\left(\partial_{v_{1}} g^{\gamma \sigma_{1}}\right)\left(\partial_{\alpha} g_{\sigma_{1} \beta}+\partial_{\beta} g_{\sigma_{1} \alpha}-\partial_{\sigma_{1}} g_{\alpha \beta}\right)+g^{\gamma \sigma_{1}}\left(\partial_{v_{1} \alpha}^{2} g_{\sigma_{1} \beta}+\partial_{\nu_{1} \beta}^{2} g_{\sigma_{1} \alpha}-\partial_{\nu_{1} \sigma_{1}}^{2} g_{\alpha \beta}\right)\right] .
\end{aligned}
$$

Thus, for $\Gamma^{\gamma}=g^{\alpha \beta} \Gamma_{\alpha \beta}^{\gamma}$,

$$
\partial_{\nu_{1}} \Gamma^{\gamma}=\left(\partial_{v_{1}} g^{\alpha \beta}\right) \Gamma_{\alpha \beta}^{\gamma}+g^{\alpha \beta}\left(\partial_{\nu_{1}} \Gamma_{\alpha \beta}^{\gamma}\right) .
$$

The swap of $\nabla$ to $\widehat{\nabla}$ in (3.10-3.12) can be reduced to a peculiar coordinate system and we now present some results concerning derivatives of quantities in normal coordinates, namely a geodesic coordinate system centered at a pinned point $x \in M$.

Let us use the notation $\stackrel{\text { n.c. }}{\longrightarrow}$ to map a quantity to its value in normal coordinates at $x$. We warn the reader that, to alleviate the presentation, we omit in the sequel the explicit dependency to $x$.

For the following results on the metric and its inverse or on the Christoffel symbols, see for instance [11, Sect. 1.11.3], $[12$, p. 5], [16] or [8].

$$
\begin{aligned}
& g_{\alpha \beta} \stackrel{\text { n.c. }}{\longrightarrow} \delta_{\alpha \beta}, \quad \partial_{v_{1}} g_{\alpha \beta} \stackrel{\text { n.c. }}{\longrightarrow} 0, \quad \partial_{v_{1} v_{2}}^{2} g_{\alpha \beta} \stackrel{\text { n.c. }}{\longrightarrow} \sum_{v_{1}, v_{2}} \frac{1}{3} R_{v_{1} \alpha v_{2} \beta}=\frac{1}{3}\left[R_{v_{1} \alpha v_{2} \beta}+R_{v_{2} \alpha v_{1} \beta}\right], \\
& \partial_{v_{1} v_{2} v_{3}}^{3} g_{\alpha \beta} \stackrel{\text { n.c. }}{\longrightarrow} \sum_{v_{1}, v_{2}, v_{3}} \frac{1}{3 !}\left(\widehat{\nabla}_{v_{1}} R\right)_{v_{2} \alpha v_{3} \beta}, \quad \partial_{v_{1} v_{2} v_{3} v_{4}}^{4} g_{\alpha \beta} \stackrel{\text { n.c. }}{\longrightarrow} \sum_{v_{1}, v_{2}, v_{3}, v_{4}}\left[\frac{1}{20}\left(\widehat{\nabla}_{v_{1} v_{2}}^{2} R\right)_{v_{3} \alpha v_{4} \beta}+\frac{2}{45} R_{v_{1} \alpha v_{2} \sigma_{1}} R_{v_{3} \beta v_{4}}{ }^{\sigma_{1}}\right]
\end{aligned}
$$

where $\sum \mathbb{v}_{v_{1}, \ldots, v_{n}} A_{v_{1} \ldots v_{n}}:=\sum_{\sigma \in \mathbb{S}_{n}} A_{v_{\sigma(1)} \ldots v_{\sigma(n)}}$ and $\mathbb{S}_{n}$ is the permutation group of $n$ elements.

Similarly,

$$
\begin{aligned}
& g^{\alpha \beta} \stackrel{\text { n.c. }}{\longrightarrow} \delta^{\alpha \beta}, \quad \partial_{\nu_{1}} g^{\alpha \beta} \stackrel{\text { n.c. }}{\longrightarrow} 0, \\
& \Gamma_{\alpha \beta}^{\gamma} \stackrel{\text { n.c. }}{\longrightarrow} 0, \quad \partial_{v_{1}} \Gamma_{\alpha \beta}^{\gamma} \stackrel{\text { n.c. }}{\longrightarrow} \frac{1}{3} \sum_{\alpha, \beta} R_{v_{1} \alpha \beta}{ }^{\gamma}=\frac{1}{3}\left[R_{v_{1} \alpha \beta}^{\gamma}+R_{v_{1} \beta \alpha}^{\gamma}\right] .
\end{aligned}
$$

Thus we obtain

$$
\begin{aligned}
& \Gamma^{\mu_{1}} \stackrel{\text { n.c. }}{\longrightarrow} 0, \quad \partial_{v_{1}} \Gamma^{\mu_{1}} \stackrel{\text { n.c. }}{\longrightarrow} \frac{2}{3} \operatorname{Ric}_{v_{1}}{ }^{\mu_{1}}, \\
& \nabla_{v_{1}} u \stackrel{\text { n.c. }}{\longrightarrow} \widehat{\nabla}_{v_{1}} u \quad \text { (equality in any coordinate system), } \\
& \nabla_{v_{1} v_{2}}^{2} u \stackrel{\text { n.c. }}{\longrightarrow} \widehat{\nabla}_{v_{1} v_{2}}^{2} u, \\
& \nabla_{v_{1}} p^{\mu_{1}} \stackrel{\text { n.c. }}{\longrightarrow} \widehat{\nabla}_{v_{1}} p^{\mu_{1}} .
\end{aligned}
$$

For $L^{\mu}$, we also get from (3.12):

$$
L^{\mu_{1}} \stackrel{\text { n.c. }}{\longrightarrow} p^{\mu_{1}}+g^{\mu_{1} \sigma_{1}}\left(\nabla_{\sigma_{1}} u\right), \quad \nabla_{v_{1}} L^{\mu_{1}} \stackrel{\text { n.c. }}{\longrightarrow} \nabla_{v_{1}} p^{\mu_{1}}+g^{\mu_{1} \sigma_{1}}\left(\nabla_{v_{1} \sigma_{1}}^{2} u\right)-\frac{2}{3} \operatorname{Ric}_{v_{1}}{ }^{\mu_{1}} u .
$$

The corresponding expressions given in terms of $N^{\mu}$ are

$$
L^{\mu_{1}} \stackrel{\text { n.c. }}{\longrightarrow} N^{\mu_{1}}, \quad \nabla_{v_{1}} L^{\mu_{1}} \stackrel{\text { n.c. }}{\longrightarrow} \nabla_{v_{1}} N^{\mu_{1}}-\frac{2}{3} \operatorname{Ric}_{v_{1}}^{\mu_{1}} u .
$$

Finally, for $H^{\mu_{1} \mu_{2}}=g^{\mu_{1} \mu_{2}} u$, we obtain directly from (3.15):

$$
H^{\mu_{1} \mu_{2}} \stackrel{\text { n.c. }}{\longrightarrow} g^{\mu_{1} \mu_{2}} u, \quad \nabla_{v_{1}} H^{\mu_{1} \mu_{2}} \stackrel{\text { n.c. }}{\longrightarrow} g^{\mu_{1} \mu_{2}}\left(\nabla_{v_{1}} u\right), \quad \nabla_{v_{1} v_{2}}^{2} H^{\mu_{1} \mu_{2}} \stackrel{\text { n.c. }}{\longrightarrow} g^{\mu_{1} \mu_{2}}\left(\nabla_{v_{1} v_{2}}^{2} u\right)-\frac{1}{3} \sum_{v_{1}, v_{2}} R_{v_{1}}{ }^{\mu_{1}}{ }_{v_{2}}{ }^{\mu_{2}} u \text {. }
$$




\section{The method and its simplifications}

In this section, we start with $h$ equal to $H(x, \xi)$, so that the definition (2.10) is specialized to the operator $f_{k}(\xi)=\pi_{\widetilde{I}_{k}}$ as seen in (2.32). With $\partial=\nabla_{v}$ acting on any local section $s$ of $V$ by $\nabla_{v} s=\partial_{v} s+A_{v} s$, so that the required Leibniz rule holds, and using (2.44), Proposition 2.11 becomes

Proposition 4.1 Given a local trivialization $s: U \rightarrow \mathbb{C}^{N}$ of a section in $\Gamma(V)$, and $B_{\ell}, 1 \leq i \leq k$, which are $k$ matrix-valued differential operators in $\nabla_{\mu}$ depending on $x$ and (linearly in) $\xi$, the following holds true:

$$
\begin{aligned}
f_{k}(\xi)\left[B_{1} \otimes \cdots \otimes B_{i} \nabla_{\nu} \otimes \cdots \otimes B_{k}\right] s= & \sum_{j=i+1}^{k} f_{k}(\xi)\left[B_{1} \otimes \cdots \otimes\left(\nabla_{v} B_{j}\right) \otimes \cdots \otimes B_{k}\right] s \\
& -\sum_{j=i}^{k} f_{k+1}(\xi)\left[B_{1} \otimes \cdots \otimes B_{j} \otimes\left(\nabla_{v} H\right) \otimes B_{j+1} \otimes \cdots \otimes B_{k}\right] s \\
& +f_{k}(\xi)\left[B_{1} \otimes \cdots \otimes B_{i} \otimes \cdots \otimes B_{k}\right]\left(\nabla_{v} s\right) .
\end{aligned}
$$

When $f_{k}(\xi)\left[B_{1} \otimes \cdots \otimes B_{k}\right]$ contains more than one covariant derivative $\nabla_{\nu}$ to propagate, they can accumulate on $s$ as $\nabla_{v_{1} \ldots v_{\ell}}^{\ell} s$, and this produces complicated expressions.

We are interested in the computation of $f_{k}(\xi)\left[B_{1} \otimes \cdots \otimes B_{k}\right]$ as a matrix-valued function, that is as a linear map: $v \in \mathbb{C}^{N} \mapsto f_{k}(\xi)\left[B_{1} \otimes \cdots \otimes B_{k}\right] v$. In that case, $\nabla_{v} v=A_{v} v$ and $\nabla_{v_{1} v_{2}}^{2} v=\left(\partial_{v_{1}} A_{v_{2}}+A_{v_{1}} A_{v_{2}}\right) v$ because $v$ is constant.

In general, we can write the result after the propagation of some $\nabla_{v}$ as a sum of terms like $f_{k^{\prime}}(\xi)\left[B_{1} \otimes \cdots \otimes B_{k^{\prime}}\right] Q[A] v$, where $Q[A]$ is a matrix-valued function written as a polynomial expression in the $A_{\mu}$ and their derivatives.

We begin the process with $f_{k}(\xi)\left[B_{1} \otimes \cdots \otimes B_{k}\right] Q_{0}[A] v$ where $Q_{0}[A]=\mathbb{1}$ (the constant unital section in End $(V)$ ) and after applying $\ell$ covariant derivatives, we get $f_{k^{\prime}}(\xi)\left[B_{1} \otimes \cdots \otimes B_{k^{\prime}}\right] Q_{\ell}[A] v$ where

$$
Q_{\ell}[A] v=\nabla_{v} Q_{\ell-1}[A] v=\left(\partial_{v} Q_{\ell-1}[A]+A_{v} Q_{\ell-1}[A]\right) v .
$$

It is easy to establish that $Q_{\ell}[A]$ is an homogeneous polynomial of degree $\ell$ when counting a degree 1 for each $\partial_{\nu}$ and $A_{v}$. In the final expression, these factors $Q[A]$ generate "gauge invariant" contributions, in term of the curvature of $A_{\mu}$ and its derivatives.

Finally, notice that Proposition 4.1 reduces to [13, Lemma 2.1] for $A_{\mu}=0$, i.e. for $\nabla_{\nu}=\partial_{\nu}$.

For $\mu_{i} \in\{1, \ldots, d\}$, let $T_{\mu_{1} \ldots \mu_{2 p}, k}$ be the operators from $\mathscr{A}^{\otimes^{k}}$ to itself defined by

$$
T_{\mu_{1} \ldots \mu_{2 p}, k}:=g_{d} G_{\mu_{1} \ldots \mu_{2 p}} I_{d / 2+p, k}\left(r_{0}, \ldots, r_{k}\right)\left(E_{0}^{R} \otimes \cdots \otimes E_{k}^{R}\right),
$$

with the notations

$$
\begin{aligned}
g_{d}(x) & :=\frac{1}{(2 \pi)^{d}} \int_{\mathbb{R}^{d}} \mathrm{~d} \xi e^{-|\xi|_{g(x)}^{2}}=\frac{|g(x)|^{1 / 2}}{2^{d} \pi^{d / 2}}, \\
G_{\mu_{1} \ldots \mu_{2 p}}(x) & :=\frac{1}{(2 \pi)^{d} g_{d}(x)} \int_{\mathbb{R}^{d}} \mathrm{~d} \xi \xi_{\mu_{1}} \cdots \xi_{\mu_{2 p}} e^{-g^{\alpha \beta}(x) \xi_{\alpha} \xi_{\beta}} \\
& =\frac{1}{2^{2 p} p !}\left(\sum_{\rho \in \mathbb{S}_{2 p}} g_{\mu_{\rho(1)} \mu_{\rho(2)}} \cdots g_{\mu_{\rho(2 p-1)} \mu_{\rho(2 p)}}\right)(x)=\frac{(2 p) !}{2^{2 p} p !} g_{\left(\mu_{1} \mu_{2}\right.} \cdots g_{\left.\mu_{2 p-1} \mu_{2 p}\right)}(x),
\end{aligned}
$$

where $\mathbb{S}_{2 p}$ is the symmetric group of permutations on $2 p$ elements and the parenthesis in the index of $g$ is the complete symmetrization over all indices and with the convention that, when $p=0, G_{\mu_{1} \ldots \mu_{2 p}}$ is just 1. For instance, $G_{\mu_{1} \mu_{2}}=\frac{1}{2} g_{\mu_{1} \mu_{2}}$ and

$$
G_{\mu_{1} \mu_{2} \mu_{3} \mu_{4}}=\frac{1}{4}\left(g_{\mu_{1} \mu_{2}} g_{\mu_{3} \mu_{4}}+g_{\mu_{1} \mu_{3}} g_{\mu_{2} \mu_{4}}+g_{\mu_{1} \mu_{4}} g_{\mu_{2} \mu_{3}}\right) .
$$

Let us also introduce the operators

$$
X_{\alpha, k, \mu_{1} \ldots \mu_{2 p}}:=G_{\mu_{1} \ldots \mu_{2 p}} X_{\alpha, k},
$$

which are justified as

$$
X_{d / 2+p, k, \mu_{1} \ldots \mu_{2 p}}=\frac{1}{g_{d}} \frac{1}{(2 \pi)^{d}} \int_{\mathbb{R}^{d}} \mathrm{~d} \xi \xi_{\mu_{1}} \cdots \xi_{\mu_{2 p}} f_{k}(\xi),
$$


because, in (2.3), $c_{k, \mu_{1}, \ldots, \mu_{2 p}}=(2 \pi)^{d} g_{d} G_{\mu_{1}, \ldots, \mu_{2 p}}$ and there is the following relations between the $f_{k}$ and the $X_{\alpha, k}$ :

$$
\begin{aligned}
\frac{1}{(2 \pi)^{d}} \int_{\mathbb{R}^{d}} \mathrm{~d} \xi \xi_{\mu_{1}} \cdots \xi_{\mu_{2 p}} f_{k}(\xi)\left[B_{1} \otimes \cdots \otimes B_{k}\right] & =\mathbf{m} \circ T_{\mu_{1} \ldots \mu_{2 p}, k}\left[B_{1} \otimes \cdots \otimes B_{k}\right]=g_{d} G_{\mu_{1} \ldots \mu_{2 p}} X_{d / 2+p, k}\left[B_{1} \otimes \cdots \otimes B_{k}\right] \\
& =g_{d} X_{d / 2+p, k, \mu_{1} \cdots \mu_{2 p}}\left[B_{1} \otimes \cdots \otimes B_{k}\right] .
\end{aligned}
$$

The appearance of $H(\xi)$ in (4.1) forces to consider the $\xi$-dependence of the arguments $B_{1} \otimes \cdots \otimes B_{k}$ : each factor depends polynomially on $\xi=\left(\xi_{1}, \ldots, \xi_{d}\right)$ because $B_{i}=\sum B^{\mu_{1} \ldots \mu_{\ell_{i}}} \xi_{\mu_{1}} \ldots \xi_{\mu_{\ell_{i}}}$ with $B^{\mu_{1} \ldots \mu_{\ell_{i}}}(x) \in M_{N}(\mathbb{C})$ independent of $\xi$.

Thus $B_{1} \otimes \cdots \otimes B_{k}$ is a sum of terms like $\xi_{\mu_{1}} \ldots \xi_{\mu_{\ell}}\left(B_{1} \otimes \cdots \otimes B_{k}\right)^{\mu_{1} \ldots \mu_{\ell}}$ and the symmetry of the $\xi$-integral implies that the ones which only survive are when $\ell=2 p$ for some $p \in \mathbb{N}$.

As a consequence, each function $a_{r}(a, P)(x)$ is expressed formally as a sum

$$
a_{r}(a, P)(x)=|g|^{-1 / 2} \sum \operatorname{tr}\left[a(x) \mathbf{m} \circ T_{\mu_{1} \ldots \mu_{2 p}, k}(x)\left[\left(B_{1}(x) \otimes \cdots \otimes B_{k}(x)\right)^{\mu_{1} \ldots \mu_{2 p}}\right]\right],
$$

and the wanted factor $\mathscr{R}_{r}$ is a sum

$$
\mathscr{R}_{r}=|g|^{-1 / 2} \sum \mathbf{m} \circ T_{\mu_{1} \ldots \mu_{2 p}, k}\left[\left(B_{1} \otimes \cdots \otimes B_{k}\right)^{\mu_{1} \ldots \mu_{2 p}}\right]=\frac{1}{(4 \pi)^{d / 2}} \sum X_{d / 2+p, k, \mu_{1} \ldots \mu_{2 p}}\left[\left(B_{1} \otimes \cdots \otimes B_{k}\right)^{\mu_{1} \ldots \mu_{2 p}}\right]
$$

because $|g|^{-1 / 2} g_{d}=\frac{1}{2^{d} \pi^{d / 2}}$.

Before we give a precise way to compute (4.8), we now store two technical lemmas

Lemma 4.2 For any $p \in \mathbb{N}^{*}, \alpha \in \mathbb{R}$ and $k \in \mathbb{N}^{*}$,

$$
\begin{aligned}
g^{\mu_{2 p-1} \mu_{2 p}} G_{\mu_{1} \ldots \mu_{2 p}} & =\left(\frac{1}{2} d+p-1\right) G_{\mu_{1} \ldots \mu_{2(p-1)}}, \\
g^{\mu_{2 p-1} \mu_{2 p}} X_{\alpha, k, \mu_{1} \ldots \mu_{2 p}} & =\left(\frac{1}{2} d+p-1\right) X_{\alpha, k, \mu_{1} \ldots \mu_{2(p-1)}} .
\end{aligned}
$$

Proof To compute the $\xi$-integration defining $G_{\mu_{1} \ldots \mu_{2 p}}$, we use spherical coordinates $\xi=s \sigma$ with $s:=\left(g^{\mu \nu} \xi_{\mu} \xi_{\nu}\right)^{1 / 2}$ and $\sigma=s^{-1} \xi \in S_{g}^{d-1}$, the unit sphere in $\left(\mathbb{R}^{d}, g\right)$. Then

$$
\begin{aligned}
g^{\mu_{2 p-1} \mu_{2 p}} G_{\mu_{1} \ldots \mu_{2 p}} & =\frac{1}{(2 \pi)^{d} g_{d}} \int_{\mathbb{R}^{d}} \mathrm{~d} \xi \xi_{\mu_{1}} \cdots \xi_{\mu_{2(p-1)}}\left(g^{\mu_{2 p-1} \mu_{2 p}} \xi_{\mu_{2 p-1}} \xi_{\mu_{2 p}}\right) e^{-g^{\alpha \beta} \xi_{\alpha} \xi_{\beta}} \\
& =\frac{1}{(2 \pi)^{d} g_{d}}\left(\int_{S_{g}^{d-1}} \mathrm{~d} \Omega_{g}(\sigma) \sigma_{\mu_{1}} \cdots \sigma_{\mu_{2 p-1}}\right)\left(\int_{0}^{\infty} \mathrm{d} s s^{d-1+2 p} e^{-s^{2}}\right) \\
& =\frac{1}{(2 \pi)^{d} g_{d}}\left(\int_{S_{g}^{d-1}}^{\mathrm{d}} \Omega_{g}(\sigma) \sigma_{\mu_{1}} \cdots \sigma_{\mu_{2 p-1}}\right) \frac{1}{2} \Gamma\left(\frac{1}{2} d+p\right) \\
& =\frac{1}{(2 \pi)^{d} g_{d}}\left(\int_{S_{g}^{d-1}} \mathrm{~d} \Omega_{g}(\sigma) \sigma_{\mu_{1}} \cdots \sigma_{\mu_{2 p-1}}\right) \frac{1}{2}\left(\frac{1}{2} d+p-1\right) \Gamma\left(\frac{1}{2} d+p-1\right) \\
& =\left(\frac{1}{2} d+p-1\right) \frac{1}{(2 \pi)^{d} g_{d}}\left(\int_{S_{g}^{d-1}} \mathrm{~d} \Omega_{g}(\sigma) \sigma_{\mu_{1}} \cdots \sigma_{\mu_{2 p-1}}\right)\left(\int_{0}^{\infty} \mathrm{d} s s^{d-1+2(p-1)} e^{-s^{2}}\right) \\
& =\left(\frac{1}{2} d+p-1\right) G_{\mu_{1} \cdots \mu_{2(p-1)}}
\end{aligned}
$$

and the equality (4.10) follows from the definition (4.5).

The full method to compute $\mathscr{R}_{r}$ consists to apply (4.1) starting from terms of the form

$$
\frac{1}{(2 \pi)^{d}} \int \mathrm{d} \xi \xi_{\mu_{1}} \cdots \xi_{\mu_{2 p}} f_{k}(\xi)\left[\left(B_{1} \otimes \cdots \otimes B_{i} \nabla_{\nu} \otimes \cdots \otimes B_{k}\right)^{\mu_{1} \cdots \mu_{2 p}}\right]
$$

generated by the series in (1.7) (the convention of Remark 1.1 is used). Considering the last term in (4.1), the most general expression to start with is (see discussion after Proposition 4.1):

$$
\frac{1}{(2 \pi)^{d}} \int \mathrm{d} \xi \xi_{\mu_{1}} \cdots \xi_{\mu_{2 p}} f_{k}(\xi)\left[\left(B_{1} \otimes \cdots \otimes B_{i} \nabla_{\nu} \otimes \cdots \otimes B_{k}\right)^{\mu_{1} \ldots \mu_{2 p}}\right] Q[A]
$$


Then the LHS of (4.1) produces three kinds of terms. The first ones come from the propagation of $\nabla$ on the arguments:

$$
\sum_{j=i+1}^{k} \frac{1}{(2 \pi)^{d}} \int \mathrm{d} \xi \xi_{\mu_{1}} \cdots \xi_{\mu_{2 p}} f_{k}(\xi)\left[\left(B_{1} \otimes \cdots \otimes\left(\nabla_{v} B_{j}\right) \otimes \cdots \otimes B_{k}\right)^{\mu_{1} \ldots \mu_{2 p}}\right] Q[A] .
$$

The second ones consist of adding $-\nabla_{v} H=-\left(\nabla_{v} H^{\alpha \beta}\right) \xi_{\alpha} \xi_{\beta}$ as an argument after $B_{i}$, so we get:

$$
-\sum_{j=i}^{k} \frac{1}{(2 \pi)^{d}} \int \mathrm{d} \xi \xi_{\mu_{1}} \cdots \xi_{\mu_{2 p}} \xi_{\alpha} \xi_{\beta} f_{k+1}(\xi)\left[\left(B_{1} \otimes \cdots \otimes B_{j} \otimes\left(\nabla_{\nu} H^{\alpha \beta}\right) \otimes B_{j+1} \otimes \cdots \otimes B_{k}\right)^{\mu_{1} \cdots \mu_{2 p}}\right] Q[A] .
$$

The third ones modify the matrix-valued polynomials $Q[A]$ as:

$$
\frac{1}{(2 \pi)^{d}} \int \mathrm{d} \xi \xi_{\mu_{1}} \cdots \xi_{\mu_{2 p}} f_{k}(\xi)\left[\left(B_{1} \otimes \cdots \otimes B_{i} \nabla_{v} \otimes \cdots \otimes B_{k}\right)^{\mu_{1} \ldots \mu_{2 p}}\right]\left(\partial_{\nu}+A_{v}\right) Q[A] .
$$

Replacing the $f_{k}$ 's and the integrations along $\xi$ with the $X_{d / 2+p, k, \mu_{1} \ldots \mu_{2 p}}$ as in (4.6), we finally obtain

$$
\begin{aligned}
X_{d / 2+p, k, \mu_{1} \ldots \mu_{2 p}}\left[\left(B_{1} \otimes \cdots \otimes\right.\right. & \left.\left.B_{i} \nabla_{\nu} \otimes \cdots \otimes B_{k}\right)^{\mu_{1} \ldots \mu_{2 p}}\right] Q[A] \\
= & +\sum_{j=i+1}^{k} X_{d / 2+p, k, \mu_{1} \ldots \mu_{2 p}}\left[\left(B_{1} \otimes \cdots \otimes\left(\nabla_{v} B_{j}\right) \otimes \cdots \otimes B_{k}\right)^{\mu_{1} \ldots \mu_{2 p}}\right] Q[A] \\
& -\sum_{j=i}^{k} X_{d / 2+p+1, k+1, \mu_{1} \ldots \mu_{2(p+1)}}\left[\left(B_{1} \otimes \cdots \otimes B_{j} \otimes\left(\nabla_{v} H^{\mu_{2 p+1} \mu_{2 p+2}}\right) \otimes \cdots \otimes B_{k}\right)^{\mu_{1} \ldots \mu_{2 p}}\right] Q[A] \\
& +X_{d / 2+p, k, \mu_{1} \ldots \mu_{2 p}}\left[\left(B_{1} \otimes \cdots \otimes B_{k}\right)^{\mu_{1} \ldots \mu_{2 p}}\right]\left(\partial_{v}+A_{v}\right) Q[A] .
\end{aligned}
$$

This relation is the "integrated" version of (4.1). One has to look inside as the tensor product over the field $\mathbb{C}$ and not over functions. In other words, it is necessary to keep the functions $g^{\alpha \beta}$ and their derivatives in front of their arguments in the tensor product until all the derivations in the arguments have propagated. Working with the matrix-valued functions $\nabla_{v} H^{\mu_{2 p+1} \mu_{2 p+2}}$ prevents this temptation.

From this result, we then get the following simplification in the computation of $\mathscr{R}_{r}$ :

Proposition 4.3 All the terms of (4.8) can be obtained starting from their analogs in (1.7) with the replacement of operators $f_{k}$ by $X_{d / 2+p, k, \mu_{1} \ldots \mu_{2 p}}$ as in (4.6) and iteratively applying the rule (4.11). At the end of this iteration, one can use (4.10) to deal with any remaining function $g^{\alpha \beta}$ together with normal coordinates to deal with derivatives of the metric.

In order to simplify the computation, we can omit the operators $X_{d / 2+p, k, \mu_{1} \ldots \mu_{2 p}}$ in (4.11) and work directly at the level of their arguments. We will also make use of two other useful symbolic notations.

Let us adopt the notation $\rightsquigarrow$ to express the development of arguments induced by (4.11). In order to take into account the presence of the matrix-valued polynomials $Q[A]$ (appearing in (4.11)) multiplied on the right of the application of $X_{d / 2+p, k, \mu_{1} \ldots \mu_{2 p}}$ on the arguments, we denote its presence with the separation symbol |, except if $Q[A]=\mathbb{1}$.

Then the computation consists to perform the propagation of all the derivatives by applying, as many times as necessary, the following symbolic rule: If $B_{i}, 1 \leq i \leq k$, are $k$ matrix-valued differential operators in $\nabla_{\mu}$ depending on $x$ and independent of $\xi$, then,

$$
\begin{aligned}
\left(B_{1} \otimes \cdots \otimes B_{i} \nabla_{v} \otimes \cdots \otimes B_{k}\right)^{\mu_{1} \ldots \mu_{2 p}} \mid Q[A] \leadsto & +\sum_{j=i+1}^{k}\left(B_{1} \otimes \cdots \otimes\left(\nabla_{\nu} B_{j}\right) \otimes \cdots \otimes B_{k}\right)^{\mu_{1} \ldots \mu_{2 p}} \mid Q[A] \\
& -\sum_{j=i}^{k}\left(B_{1} \otimes \cdots \otimes B_{j} \otimes\left(\nabla_{v} H^{\mu_{2 p+1} \mu_{2 p+2}}\right) \otimes B_{j+1} \otimes \cdots \otimes B_{k}\right)^{\mu_{1} \cdots \mu_{2 p}} \mid Q[A] \\
& +\left(B_{1} \otimes \cdots \otimes B_{i} \otimes \cdots \otimes B_{k}\right)^{\mu_{1} \cdots \mu_{2 p}} \mid \nabla_{\nu} Q[A] .
\end{aligned}
$$

Once this rule has been used, the operators $X_{d / 2+p, k, \mu_{1} \ldots \mu_{2 p}}$ to apply on each argument in the obtained sum are uniquely determined by the number of free indices $\mu_{i}$ and the number of arguments in tensor products. 
Remark 4.4 When $u=\mathbb{1}$, the previous formula (4.12) cannot be simplified because $H^{\mu v}=g^{\mu v} \mathbb{1}$ commutes with matrixvalued functions but not with some differential operators $\nabla_{v}$ possibly contained in some $B_{\ell}$ for $1 \leq \ell \leq i$. This implies in particular that we can not hope for simplifications at this computation stage even if $u=\mathbb{1}$, and the number of terms produced by successive applications of (4.12) is independent of the exact form of $H^{\mu \nu}$. Only subsequent computations can use some hypothesis on $u$ for simplifications.

Remark 4.5 It is tempting in the previous method to start with $P$ written in terms of $\widehat{\nabla}$ as in (3.8) and to propagate $\widehat{\nabla}_{\mu}$ instead of $\nabla_{\mu}$. But this requires to get an analogue of formula (1.2) which needs to make sense of $\widehat{\nabla}_{\mu} \xi_{\nu}$ : the variables $\xi_{\nu}$ are the Fourier dual of the variables $x^{v}$ and, even if they carry a space index, they are not the component of a tensor field on $M$. While here these variables $\xi_{v}$ are silent since only confined in the $G$-tensors (as a consequence of $\nabla_{\mu} \xi_{v}=0$ ), they would have to remain both in the $B_{j}$ and $H^{\mu v}$ to be sensitive to the action of $\widehat{\nabla}$ in (4.12), thus generating more terms. This would give directly the result in terms of $\widehat{\nabla}$ while here, we are obliged to exchange with some efforts the $\nabla$ with $\widehat{\nabla}$ (in normal coordinates) at the end.

\section{Results on $\mathscr{R}_{2}$}

This section is devoted to a complete computation of $\mathscr{R}_{2}$ via the above method and is a more algebraic version than the similar result obtained in [14, Thm 2.4].

We plane to follow Proposition 4.3 to compute $\mathscr{R}_{2}$ in $a_{2}(a, P)(x)=\operatorname{tr}\left[a(x) \mathscr{R}_{2}(x)\right]$ and start with

$$
(4 \pi)^{d / 2} \mathscr{R}_{2}=X_{d / 2+1,2, \mu_{1} \mu_{2}}\left[K^{\mu_{1}} \otimes K^{\mu_{2}}\right]-X_{d / 2,1}[P] .
$$

We perform the computation at the level of arguments using (4.12). Notice that all the used spectral operators $X_{\alpha, k}$ (and those appearing in the final result) are in the series $X_{d / 2,1}, X_{d / 2+1,2}, \ldots, X_{d / 2+k-1, k}$.

Consider first $-X_{d / 2,1}[P]$ :

$$
\begin{aligned}
-P= & H^{v_{1} v_{2}} \nabla_{v_{1} v_{2}}^{2}+L^{v_{1}} \nabla_{v_{1}}+q \\
\rightsquigarrow & -H^{v_{1} v_{2}} \nabla_{v_{1}} \otimes\left(\nabla_{v_{2}} H^{\mu_{1} \mu_{2}}\right)+H^{v_{1} v_{2}} \nabla_{v_{1}}\left|A_{v_{2}}-L^{v_{1}} \otimes\left(\nabla_{v_{1}} H^{\mu_{1} \mu_{2}}\right)+L^{v_{1}}\right| A_{v_{2}}+q \\
\rightsquigarrow & -H^{v_{1} v_{2}} \otimes\left(\nabla_{v_{1} v_{2}}^{2} H^{\mu_{1} \mu_{2}}\right)+H^{v_{1} v_{2}} \otimes\left(\nabla_{v_{1}} H^{\mu_{3} \mu_{4}}\right) \otimes\left(\nabla_{v_{2}} H^{\mu_{1} \mu_{2}}\right)+H^{v_{1} v_{2}} \otimes\left(\nabla_{v_{2}} H^{\mu_{1} \mu_{2}}\right) \otimes\left(\nabla_{v_{1}} H^{\mu_{3} \mu_{4}}\right) \\
& -H^{v_{1} v_{2}} \otimes\left(\nabla_{v_{2}} H^{\mu_{1} \mu_{2}}\right)\left|A_{v_{1}}-H^{v_{1} v_{2}} \otimes\left(\nabla_{v_{1}} H^{\mu_{1} \mu_{2}}\right)\right| A_{v_{2}}+H^{v_{1} v_{2}} \mid\left(\partial_{v_{1}} A_{v_{2}}+A_{v_{1}} A_{v_{2}}\right) \\
& \quad-L^{v_{1}} \otimes\left(\nabla_{v_{1}} H^{\mu_{1} \mu_{2}}\right)+L^{v_{1}} \mid A_{v_{1}}+q \\
\stackrel{\text { n.c. }}{\longrightarrow} & -g^{v_{1} v_{2}} g^{\mu_{1} \mu_{2}} u \otimes\left(\nabla_{v_{1} v_{2}}^{2} u\right)+\frac{1}{3} g^{v_{1} v_{2}}\left(\sum_{v_{1}, v_{2}} R_{v_{1}}^{\mu_{1}} v_{v_{2}}^{\mu_{2}}\right) u \otimes u+2 g^{v_{1} v_{2}} g^{\mu_{1} \mu_{2}} g^{\mu_{3} \mu_{4}} u \otimes\left(\nabla_{v_{1}} u\right) \otimes\left(\nabla_{v_{2}} u\right) \\
& -2 g^{v_{1} v_{2}} g^{\mu_{1} \mu_{2}} u \otimes\left(\nabla_{v_{1}} u\right)\left|A_{v_{2}}+g^{v_{1} v_{2}} u\right|\left(\partial_{v_{1}} A_{v_{2}}+A_{v_{1}} A_{v_{2}}\right) \\
& -g^{\mu_{1} \mu_{2}} p^{v_{1}} \otimes\left(\nabla_{v_{1}} u\right)-g^{v_{1} \sigma_{1}} g^{\mu_{1} \mu_{2}}\left(\nabla_{\sigma_{1}} u\right) \otimes\left(\nabla_{v_{1}} u\right)+L^{v_{1}} \mid A_{v_{1}}+q .
\end{aligned}
$$

Similarly for $X_{d / 2+1,2, \mu_{1} \mu_{2}}\left[K^{\mu_{1}} \otimes K^{\mu_{2}}\right]$ :

$$
\begin{aligned}
& K^{\mu_{1}} \otimes K^{\mu_{2}}=-\left(L^{\mu_{1}}+2 H^{\mu_{1} v_{1}} \nabla_{v_{1}}\right) \otimes\left(L^{\mu_{2}}+2 H^{\mu_{2} v_{2}} \nabla_{v_{2}}\right) \\
& =-L^{\mu_{1}} \otimes L^{\mu_{2}}-2 L^{\mu_{1}} \otimes H^{\mu_{2} v_{2}} \nabla_{v_{2}}-2 H^{\mu_{1} v_{1}} \nabla_{v_{1}} \otimes L^{\mu_{2}}-4 H^{\mu_{1} v_{1}} \nabla_{v_{1}} \otimes H^{\mu_{2} v_{2}} \nabla_{v_{2}} \\
& \rightsquigarrow-L^{\mu_{1}} \otimes L^{\mu_{2}}+2 L^{\mu_{1}} \otimes H^{\mu_{2} v_{2}} \otimes\left(\nabla_{v_{2}} H^{\mu_{3} \mu_{4}}\right)-2 L^{\mu_{1}} \otimes H^{\mu_{2} v_{2}} \mid A_{v_{2}} \\
& -2 H^{\mu_{1} v_{1}} \otimes\left(\nabla_{v_{1}} L^{\mu_{2}}\right)+2 H^{\mu_{1} v_{1}} \otimes\left(\nabla_{v_{1}} H^{\mu_{3} \mu_{4}}\right) \otimes L^{\mu_{2}} \\
& +2 H^{\mu_{1} v_{1}} \otimes L^{\mu_{2}} \otimes\left(\nabla_{v_{1}} H^{\mu_{3} \mu_{4}}\right)-2 H^{\mu_{1} v_{1}} \otimes L^{\mu_{2}} \mid A_{v_{1}} \\
& +4 H^{\mu_{1} v_{1}} \nabla_{v_{1}} \otimes H^{\mu_{2} v_{2}} \otimes\left(\nabla_{v_{2}} H^{\mu_{3} \mu_{4}}\right)-4 H^{\mu_{1} v_{1}} \nabla_{v_{1}} \otimes H^{\mu_{2} v_{2}} \mid A_{v_{2}} \\
& \rightsquigarrow-L^{\mu_{1}} \otimes L^{\mu_{2}}+2 L^{\mu_{1}} \otimes H^{\mu_{2} v_{2}} \otimes\left(\nabla_{v_{2}} H^{\mu_{3} \mu_{4}}\right)-2 L^{\mu_{1}} \otimes H^{\mu_{2} v_{2}} \mid A_{v_{2}} \\
& -2 H^{\mu_{1} v_{1}} \otimes\left(\nabla_{v_{1}} L^{\mu_{2}}\right)+2 H^{\mu_{1} v_{1}} \otimes\left(\nabla_{v_{1}} H^{\mu_{3} \mu_{4}}\right) \otimes L^{\mu_{2}}+2 H^{\mu_{1} v_{1}} \otimes L^{\mu_{2}} \otimes\left(\nabla_{v_{1}} H^{\mu_{3} \mu_{4}}\right)-2 H^{\mu_{1} v_{1}} \otimes L^{\mu_{2}} \mid A_{v_{1}} \\
& +4 H^{\mu_{1} v_{1}} \otimes\left(\nabla_{v_{1}} H^{\mu_{2} v_{2}}\right) \otimes\left(\nabla_{v_{2}} H^{\mu_{3} \mu_{4}}\right)+4 H^{\mu_{1} v_{1}} \otimes H^{\mu_{2} v_{2}} \otimes\left(\nabla_{v_{1} v_{2}}^{2} H^{\mu_{3} \mu_{4}}\right) \\
& -4 H^{\mu_{1} v_{1}} \otimes\left(\nabla_{v_{1}} H^{\mu_{5} \mu_{6}}\right) \otimes H^{\mu_{2} v_{2}} \otimes\left(\nabla_{v_{2}} H^{\mu_{3} \mu_{4}}\right)-4 H^{\mu_{1} v_{1}} \otimes H^{\mu_{2} v_{2}} \otimes\left(\nabla_{v_{1}} H^{\mu_{5} \mu_{6}}\right) \otimes\left(\nabla_{v_{2}} H^{\mu_{3} \mu_{4}}\right) \\
& -4 H^{\mu_{1} v_{1}} \otimes H^{\mu_{2} v_{2}} \otimes\left(\nabla_{v_{2}} H^{\mu_{3} \mu_{4}}\right) \otimes\left(\nabla_{v_{1}} H^{\mu_{5} \mu_{6}}\right)+4 H^{\mu_{1} v_{1}} \otimes H^{\mu_{2} v_{2}} \otimes\left(\nabla_{v_{2}} H^{\mu_{3} \mu_{4}}\right) \mid A_{v_{1}} \\
& -4 H^{\mu_{1} v_{1}} \otimes\left(\nabla_{v_{1}} H^{\mu_{2} v_{2}}\right)\left|A_{v_{2}}+4 H^{\mu_{1} v_{1}} \otimes\left(\nabla_{v_{1}} H^{\mu_{3} \mu_{4}}\right) \otimes H^{\mu_{2} v_{2}}\right| A_{v_{2}} \\
& +4 H^{\mu_{1} v_{1}} \otimes H^{\mu_{2} v_{2}} \otimes\left(\nabla_{v_{1}} H^{\mu_{3} \mu_{4}}\right)\left|A_{v_{2}}-4 H^{\mu_{1} v_{1}} \otimes H^{\mu_{2} v_{2}}\right|\left(\partial_{v_{1}} A_{v_{2}}+A_{v_{1}} A_{v_{2}}\right)
\end{aligned}
$$




$$
\begin{aligned}
& \stackrel{\text { n.c. }}{\longrightarrow}-p^{\mu_{1}} \otimes p^{\mu_{2}}-g^{\mu_{2} \sigma_{2}} p^{\mu_{1}} \otimes\left(\nabla_{\sigma_{2}} u\right)-g^{\mu_{1} \sigma_{1}}\left(\nabla_{\sigma_{1}} u\right) \otimes p^{\mu_{2}}-g^{\mu_{1} \sigma_{1}} g^{\mu_{2} \sigma_{2}}\left(\nabla_{\sigma_{1}} u\right) \otimes\left(\nabla_{\sigma_{2}} u\right) \\
& +2 g^{\mu_{2} v_{2}} g^{\mu_{3} \mu_{4}} p^{\mu_{1}} \otimes u \otimes\left(\nabla_{v_{2}} u\right)+2 g^{\mu_{1} \sigma_{1}} g^{\mu_{2} v_{2}} g^{\mu_{3} \mu_{4}}\left(\nabla_{\sigma_{1}} u\right) \otimes u \otimes\left(\nabla_{v_{2}} u\right)-2 g^{\mu_{2} v_{2}} L^{\mu_{1}} \otimes u \mid A_{v_{2}} \\
& -2 g^{\mu_{1} v_{1}} u \otimes\left(\nabla_{v_{1}} p^{\mu_{2}}\right)-2 g^{\mu_{1} v_{1}} g^{\mu_{2} \sigma_{2}} u \otimes\left(\nabla_{v_{1} \sigma_{2}}^{2} u\right)+\frac{4}{3} g^{\mu_{1} v_{1}} \operatorname{Ric}_{v_{1}}^{\mu_{2}} u \otimes u \\
& +2 g^{\mu_{1} v_{1}} g^{\mu_{3} \mu_{4}} u \otimes\left(\nabla_{v_{1}} u\right) \otimes p^{\mu_{2}}+2 g^{\mu_{1} v_{1}} g^{\mu_{3} \mu_{4}} g^{\mu_{2} \sigma_{2}} u \otimes\left(\nabla_{v_{1}} u\right) \otimes\left(\nabla_{\sigma_{2}} u\right) \\
& +2 g^{\mu_{1} v_{1}} g^{\mu_{3} \mu_{4}} u \otimes p^{\mu_{2}} \otimes\left(\nabla_{v_{1}} u\right)+2 g^{\mu_{1} v_{1}} g^{\mu_{2} \sigma_{2}} g^{\mu_{3} \mu_{4}} u \otimes\left(\nabla_{\sigma_{2}} u\right) \otimes\left(\nabla_{v_{1}} u\right)-2 g^{\mu_{1} v_{1}} u \otimes L^{\mu_{2}} \mid A_{v_{1}} \\
& +4 g^{\mu_{1} \nu_{1}} g^{\mu_{2} \nu_{2}} g^{\mu_{3} \mu_{4}} u \otimes\left(\nabla_{v_{1}} u\right) \otimes\left(\nabla_{v_{2}} u\right)+4 g^{\mu_{1} v_{1}} g^{\mu_{2} \nu_{2}} g^{\mu_{3} \mu_{4}} u \otimes u \otimes\left(\nabla_{v_{1} v_{2}}^{2} u\right) \\
& -\frac{4}{3} g^{\mu_{1} v_{1}} g^{\mu_{2} \nu_{2}}\left(\sum_{v_{1}, v_{2}} R_{v_{1}}^{\mu_{3}}{ }_{v_{2}}^{\mu_{4}}\right) u \otimes u \otimes u-4 g^{\mu_{1} v_{1}} g^{\mu_{5} \mu_{6}} g^{\mu_{2} v_{2}} g^{\mu_{3} \mu_{4}} u \otimes\left(\nabla_{v_{1}} u\right) \otimes u \otimes\left(\nabla_{v_{2}} u\right) \\
& -4 g^{\mu_{1} v_{1}} g^{\mu_{2} \nu_{2}} g^{\mu_{5} \mu_{6}} g^{\mu_{3} \mu_{4}} u \otimes u \otimes\left(\nabla_{v_{1}} u\right) \otimes\left(\nabla_{v_{2}} u\right)-4 g^{\mu_{1} \nu_{1}} g^{\mu_{2} v_{2}} g^{\mu_{3} \mu_{4}} g^{\mu_{5} \mu_{6}} u \otimes u \otimes\left(\nabla_{v_{2}} u\right) \otimes\left(\nabla_{v_{1}} u\right) \\
& +4 g^{\mu_{1} v_{1}} g^{\mu_{2} v_{2}} g^{\mu_{3} \mu_{4}} u \otimes u \otimes\left(\nabla_{v_{2}} u\right)\left|A_{v_{1}}-4 g^{\mu_{1} v_{1}} g^{\mu_{2} v_{2}} u \otimes\left(\nabla_{v_{1}} u\right)\right| A_{v_{2}} \\
& +4 g^{\mu_{1} v_{1}} g^{\mu_{3} \mu_{4}} g^{\mu_{2} v_{2}} u \otimes\left(\nabla_{v_{1}} u\right) \otimes u\left|A_{v_{2}}+4 g^{\mu_{1} v_{1}} g^{\mu_{2} v_{2}} g^{\mu_{3} \mu_{4}} u \otimes u \otimes\left(\nabla_{v_{1}} u\right)\right| A_{v_{2}} \\
& -4 g^{\mu_{1} v_{1}} g^{\mu_{2} v_{2}} u \otimes u \mid\left(\partial_{v_{1}} A_{v_{2}}+A_{v_{1}} A_{v_{2}}\right) \text {. }
\end{aligned}
$$

We can now apply the operators $X_{d / 2,1}, X_{d / 2+1,2, \mu_{1} \mu_{2}}, X_{d / 2+2,3, \mu_{1} \mu_{2} \mu_{3} \mu_{4}}$ and $X_{d / 2+3,4, \mu_{1} \mu_{2} \mu_{3} \mu_{4} \mu_{5} \mu_{6}}$ according to the obtained arguments, use (4.10) when necessary, and finally collect all the terms.

Let us first collect the terms with $Q[A] \neq \mathbb{1}$ and show that they all cancel thanks to (2.20).

The terms with $\left(\partial_{v_{1}} A_{v_{2}}+A_{v_{1}} A_{v_{2}}\right)$ do not contribute:

$$
g^{v_{1} v_{2}} X_{d / 2,1}[u]-4 g^{\mu_{1} v_{1}} g^{\mu_{2} v_{2}} X_{d / 2+1,2, \mu_{1} \mu_{2}}[u \otimes u]=g^{v_{1} v_{2}}\left(X_{d / 2,1}[u]-2 X_{d / 2+1,2}[u \otimes u]\right)=0 .
$$

The sum of terms containing $L^{\mu}$ and $A_{\nu}$ vanishes:

$$
\begin{aligned}
\left(X_{d / 2,1}\left[L^{v_{1}}\right]-2 g^{\mu_{2} v_{1}} X_{d / 2+1,2, \mu_{1} \mu_{2}}\left[L^{\mu_{1}} \otimes u\right]-2 g^{\mu_{1} v_{1}} X_{d / 2+1,2, \mu_{1} \mu_{2}}\left[u \otimes L^{\mu_{2}}\right]\right) A_{v_{1}} \\
\\
=\left(X_{d / 2,1}\left[L^{v_{1}}\right]-X_{d / 2+1,2}\left[L^{v_{1}} \otimes u\right]-X_{d / 2+1,2}\left[u \otimes L^{v_{1}}\right]\right) A_{v_{1}}=0 .
\end{aligned}
$$

Finally the contribution of terms containing $\nabla_{v} u$ and $A_{v}$ also vanishes:

$$
\begin{aligned}
& \left(-2 g^{v_{1} \nu_{2}} g^{\mu_{1} \mu_{2}} X_{d / 2+1,2, \mu_{1} \mu_{2}}\left[u \otimes \nabla_{v_{1}} u\right]-4 g^{\mu_{1} v_{1}} g^{\mu_{2} \nu_{2}} X_{d / 2+1,2, \mu_{1} \mu_{2}}\left[u \otimes \nabla_{v_{1}} u\right]\right. \\
& \left.+4 g^{\mu_{1} v_{1}} g^{\mu_{2} v_{2}} g^{\mu_{3} \mu_{4}} X_{d / 2+2,3, \mu_{1} \mu_{2} \mu_{3} \mu_{4}}\left[u \otimes \nabla_{v_{1}} u \otimes u\right]+8 g^{\mu_{1} v_{1}} g^{\mu_{2} v_{2}} g^{\mu_{3} \mu_{4}} X_{d / 2+2,3, \mu_{1} \mu_{2} \mu_{3} \mu_{4}}\left[u \otimes u \otimes \nabla_{v_{1}} u\right]\right) A_{v_{2}} \\
& \quad=g^{\nu_{1} v_{2}}(d+2)\left(-X_{d / 2+1,2}\left[u \otimes \nabla_{v_{1}} u\right]+X_{d / 2+2,3}\left[u \otimes \nabla_{v_{1}} u \otimes u\right]+2 X_{d / 2+2,3}\left[u \otimes u \otimes \nabla_{v_{1}} u\right]\right) A_{v_{2}}=0 .
\end{aligned}
$$

There are 3 terms which contain tensor products of $u$ only:

$$
\begin{aligned}
& +\frac{1}{3} g^{\nu_{1} v_{2}}\left(\sum_{v_{1}, v_{2}} R_{v_{1}}^{\mu_{1}}{ }_{v_{2}}^{\mu_{2}}\right) X_{d / 2+1,2, \mu_{1} \mu_{2}}[u \otimes u]=\frac{1}{6} g_{\mu_{1} \mu_{2}} g^{v_{1} v_{2}}\left(\sum_{v_{1}, v_{2}} R_{v_{1}}{ }^{\mu_{1}}{ }_{v_{2}}^{\mu_{2}}\right) X_{d / 2+1,2}[u \otimes u]=-\frac{1}{3} \Re X_{d / 2+1,2}[u \otimes u], \\
& +\frac{4}{3} g^{\mu_{1} v_{1}} \operatorname{Ric}_{v_{1}}^{\mu_{2}} X_{d / 2+1,2, \mu_{1} \mu_{2}}[u \otimes u]=\frac{2}{3} g_{\mu_{1} \mu_{2}} g^{\mu_{1} v_{1}} \operatorname{Ric}_{v_{1}}{ }^{\mu_{2}} X_{d / 2+1,2}[u \otimes u]=\frac{2}{3} \Re X_{d / 2+1,2}[u \otimes u], \\
& -\frac{4}{3} g^{\mu_{1} v_{1}} g^{\mu_{2} v_{2}}\left(\sum_{v_{1}, v_{2}} R_{v_{1}}{ }^{\mu_{3}}{ }_{v_{2}}^{\mu_{4}}\right) X_{d / 2+2,3, \mu_{1} \mu_{2} \mu_{3} \mu_{4}}[u \otimes u \otimes u]=-\frac{4}{3} G_{\mu_{1} \mu_{2} \mu_{3} \mu_{4}}\left(\sum_{\mu_{1}, \mu_{2}} R^{\mu_{1} \mu_{3} \mu_{2} \mu_{4}}\right) X_{d / 2+2,3}[u \otimes u \otimes u]=0,
\end{aligned}
$$

by the complete symmetry of $G_{\mu_{1} \mu_{2} \mu_{3} \mu_{4}}$ and the skew symmetry of the first and second couples of indices in $R^{\mu_{1} \mu_{3} \mu_{2} \mu_{4}}$. Since by (2.16), $X_{d / 2+1,2}[u \otimes u]=\frac{1}{2} u^{-d / 2+1}$, this amounts to $\frac{1}{6} \mathfrak{R} u^{-d / 2+1}=\frac{1}{6} \mathfrak{R} X_{d / 2,1}[u]$.

The only term in $q$ is $X_{d / 2,1}[q]$.

The sum of 3 terms containing $\nabla_{v_{1} v_{2}}^{2} u$ is

$$
\begin{aligned}
& -g^{v_{1} v_{2}} g^{\mu_{1} \mu_{2}} X_{d / 2+1,2, \mu_{1} \mu_{2}}\left[u \otimes \nabla_{v_{1} v_{2}}^{2} u\right]-2 g^{\mu_{1} \nu_{1}} g^{\mu_{2} v_{2}} X_{d / 2+1,2, \mu_{1} \mu_{2}}\left[u \otimes \nabla_{v_{1} v_{2}}^{2} u\right] \\
& +4 g^{\mu_{1} v_{1}} g^{\mu_{2} v_{2}} g^{\mu_{3} \mu_{4}} X_{d / 2+2,3, \mu_{1} \mu_{2} \mu_{3} \mu_{4}}\left[u \otimes u \otimes \nabla_{v_{1} v_{2}}^{2} u\right] \\
& =-\frac{1}{2} d g^{\nu_{1} v_{2}} X_{d / 2+1,2}\left[u \otimes \nabla_{v_{1} v_{2}}^{2} u\right]-g^{v_{1} v_{2}} X_{d / 2+1,2}\left[u \otimes \nabla_{v_{1} v_{2}}^{2} u\right] \\
& +(d+2) g^{\nu_{1} v_{2}} X_{d / 2+2,3}\left[u \otimes u \otimes \nabla_{v_{1} v_{2}}^{2} u\right] \\
& =-\frac{1}{2}(d+2) g^{v_{1} v_{2}} X_{d / 2+1,2}\left[u \otimes \nabla_{v_{1} \nu_{2}}^{2} u\right]+(d+2) g^{v_{1} v_{2}} X_{d / 2+2,3}\left[u \otimes u \otimes \nabla_{v_{1} v_{2}}^{2} u\right] \text {. }
\end{aligned}
$$


The sum of 10 terms in $\nabla_{v_{1}} u$ and $\nabla_{v_{2}} u$ is:

$$
\begin{aligned}
& 2 g^{\nu_{1} \nu_{2}} g^{\mu_{1} \mu_{2}} g^{\mu_{3} \mu_{4}} X_{d / 2+2,3, \mu_{1} \mu_{2} \mu_{3} \mu_{4}}\left[u \otimes \nabla_{v_{1}} u \otimes \nabla_{v_{2}} u\right]-g^{v_{1} \nu_{2}} g^{\mu_{1} \mu_{2}} X_{d / 2+1,2, \mu_{1} \mu_{2}}\left[\nabla_{v_{1}} u \otimes \nabla_{v_{2}} u\right] \\
& -g^{\mu_{1} v_{1}} g^{\mu_{2} v_{2}} X_{d / 2+1,2, \mu_{1} \mu_{2}}\left[\nabla_{v_{1}} u \otimes \nabla_{v_{2}} u\right]+2 g^{\mu_{1} v_{1}} g^{\mu_{2} v_{2}} g^{\mu_{3} \mu_{4}} X_{d / 2+2,3, \mu_{1} \mu_{2} \mu_{3} \mu_{4}}\left[\nabla_{v_{1}} u \otimes u \otimes \nabla_{v_{2}} u\right] \\
& +2 g^{\mu_{1} v_{1}} g^{\mu_{2} v_{2}} g^{\mu_{3} \mu_{4}} X_{d / 2+2,3, \mu_{1} \mu_{2} \mu_{3} \mu_{4}}\left[u \otimes \nabla_{v_{1}} u \otimes \nabla_{v_{2}} u\right]+2 g^{\mu_{1} v_{1}} g^{\mu_{2} v_{2}} g^{\mu_{3} \mu_{4}} X_{d / 2+2,3, \mu_{1} \mu_{2} \mu_{3} \mu_{4}}\left[u \otimes \nabla_{v_{2}} u \otimes \nabla_{v_{1}} u\right] \\
& +4 g^{\mu_{1} v_{1}} g^{\mu_{2} v_{2}} g^{\mu_{3} \mu_{4}} X_{d / 2+2,3, \mu_{1} \mu_{2} \mu_{3} \mu_{4}}\left[u \otimes \nabla_{v_{1}} u \otimes \nabla_{v_{2}} u\right] \\
& -4 g^{\mu_{1} v_{1}} g^{\mu_{2} v_{2}} g^{\mu_{3} \mu_{4}} g^{\mu_{5} \mu_{6}} X_{d / 2+3,4, \mu_{1} \mu_{2} \mu_{3} \mu_{4} \mu_{5} \mu_{6}}\left[u \otimes \nabla_{v_{1}} u \otimes u \otimes \nabla_{v_{2}} u\right] \\
& -4 g^{\mu_{1} v_{1}} g^{\mu_{2} v_{2}} g^{\mu_{3} \mu_{4}} g^{\mu_{5} \mu_{6}} X_{d / 2+3,4, \mu_{1} \mu_{2} \mu_{3} \mu_{4} \mu_{5} \mu_{6}}\left[u \otimes u \otimes \nabla_{v_{1}} u \otimes \nabla_{v_{2}} u\right]
\end{aligned}
$$

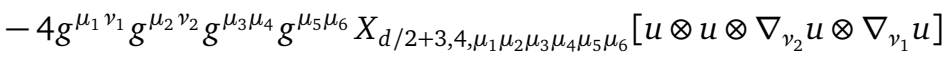

$$
\begin{aligned}
& =\frac{1}{2} d(d+2) g^{\nu_{1} v_{2}} X_{d / 2+2,3}\left[u \otimes \nabla_{v_{1}} u \otimes \nabla_{v_{2}} u\right]-\frac{1}{2} d g^{\nu_{1} v_{2}} X_{d / 2+1,2}\left[\nabla_{\nu_{1}} u \otimes \nabla_{v_{2}} u\right] \\
& -\frac{1}{2} g^{v_{1} v_{2}} X_{d / 2+1,2}\left[\nabla_{v_{1}} u \otimes \nabla_{v_{2}} u\right]+\frac{1}{2}(d+2) g^{v_{1} v_{2}} X_{d / 2+2,3}\left[\nabla_{v_{1}} u \otimes u \otimes \nabla_{v_{2}} u\right] \\
& +2(d+2) g^{v_{1} v_{2}} X_{d / 2+2,3}\left[u \otimes \nabla_{v_{1}} u \otimes \nabla_{v_{2}} u\right]-\frac{1}{2}(d+2)(d+4) g^{v_{1} v_{2}} X_{d / 2+3,4}\left[u \otimes \nabla_{v_{1}} u \otimes u \otimes \nabla_{v_{2}} u\right] \\
& -(d+2)(d+4) g^{v_{1} v_{2}} X_{d / 2+3,4}\left[u \otimes u \otimes \nabla_{v_{1}} u \otimes \nabla_{v_{2}} u\right] \\
& =-\frac{1}{2}(d+1) g^{v_{1} v_{2}} X_{d / 2+1,2}\left[\nabla_{v_{1}} u \otimes \nabla_{v_{2}} u\right]+\frac{1}{2}(d+2)(d+4) g^{v_{1} v_{2}} X_{d / 2+2,3}\left[u \otimes \nabla_{v_{1}} u \otimes \nabla_{v_{2}} u\right] \\
& +\frac{1}{2}(d+2) g^{v_{1} v_{2}} X_{d / 2+2,3}\left[\nabla_{v_{1}} u \otimes u \otimes \nabla_{v_{2}} u\right]-\frac{1}{2}(d+2)(d+4) g^{\nu_{1} v_{2}} X_{d / 2+3,4}\left[u \otimes \nabla_{v_{1}} u \otimes u \otimes \nabla_{v_{2}} u\right] \\
& -(d+2)(d+4) g^{v_{1} v_{2}} X_{d / 2+3,4}\left[u \otimes u \otimes \nabla_{\nu_{1}} u \otimes \nabla_{v_{2}} u\right] \text {. }
\end{aligned}
$$

The only term in $\nabla_{v} p^{\mu}$ is

$$
-2 g^{\mu_{1} v_{1}} X_{d / 2+1,2, \mu_{1} \mu_{2}}\left[u \otimes \nabla_{v_{1}} p^{\mu_{2}}\right]=-X_{d / 2+1,2}\left[u \otimes \nabla_{\mu} p^{\mu}\right] .
$$

The 4 terms in $p^{\mu}$ and $\nabla_{v} u$ are

$$
\begin{aligned}
& -g^{\mu_{1} \mu_{2}} X_{d / 2+1,2, \mu_{1} \mu_{2}}\left[p^{v_{1}} \otimes \nabla_{v_{1}} u\right]-g^{\mu_{2} v_{1}} X_{d / 2+1,2, \mu_{1} \mu_{2}}\left[p^{\mu_{1}} \otimes \nabla_{v_{1}} u\right] \\
& +2 g^{\mu_{2} v_{2}} g^{\mu_{3} \mu_{4}} X_{d / 2+2,3, \mu_{1} \mu_{2} \mu_{3} \mu_{4}}\left[p^{\mu_{1}} \otimes u \otimes \nabla_{v_{2}} u\right]+2 g^{\mu_{1} v_{1}} g^{\mu_{3} \mu_{4}} X_{d / 2+2,3, \mu_{1} \mu_{2} \mu_{3} \mu_{4}}\left[u \otimes p^{\mu_{2}} \otimes \nabla_{v_{1}} u\right] \\
& =-\frac{1}{2} d X_{d / 2+1,2}\left[p^{v_{1}} \otimes \nabla_{v_{1}} u\right]-\frac{1}{2} X_{d / 2+1,2}\left[p^{v_{1}} \otimes \nabla_{v_{1}} u\right] \\
& \quad \quad \quad \frac{1}{2}(d+2) X_{d / 2+2,3}\left[p^{v_{1}} \otimes u \otimes \nabla_{v_{1}} u\right]+\frac{1}{2}(d+2) X_{d / 2+2,3}\left[u \otimes p^{v_{1}} \otimes \nabla_{v_{1}} u\right] \\
& \quad=-\frac{1}{2}(d+1) X_{d / 2+1,2}\left[p^{v_{1}} \otimes \nabla_{v_{1}} u\right]+\frac{1}{2}(d+2) X_{d / 2+2,3}\left[p^{v_{1}} \otimes u \otimes \nabla_{v_{1}} u\right]+\frac{1}{2}(d+2) X_{d / 2+2,3}\left[u \otimes p^{v_{1}} \otimes \nabla_{\nu_{1}} u\right] .
\end{aligned}
$$

The 2 terms in $\nabla_{v} u$ and $p^{\mu}$ are

$$
\begin{gathered}
-g^{\mu_{1} \nu_{1}} X_{d / 2+1,2, \mu_{1} \mu_{2}}\left[\nabla_{v_{1}} u \otimes p^{\mu_{2}}\right]+2 g^{\mu_{1} \nu_{1}} g^{\mu_{3} \mu_{4}} X_{d / 2+2,3, \mu_{1} \mu_{2} \mu_{3} \mu_{4}}\left[u \otimes \nabla_{v_{1}} u \otimes p^{\mu_{2}}\right] \\
=-\frac{1}{2} X_{d / 2+1,2}\left[\nabla_{v_{1}} u \otimes p^{v_{1}}\right]+\frac{1}{2}(d+2) X_{d / 2+2,3}\left[u \otimes \nabla_{v_{1}} u \otimes p^{v_{1}}\right] .
\end{gathered}
$$

Finally, the term in $p^{\mu_{1}}$ and $p^{\mu_{2}}$ is

$$
-X_{d / 2+1,2, \mu_{1} \mu_{2}}\left[p^{\mu_{1}} \otimes p^{\mu_{2}}\right]=-\frac{1}{2} g_{\mu_{1} \mu_{2}} X_{d / 2+1,2}\left[p^{\mu_{1}} \otimes p^{\mu_{2}}\right] .
$$

Since the computation has been performed in normal coordinates, using (3.16), (3.17) and (3.18), one can replace the gauge covariant derivative $\nabla_{\mu}$ by the total derivative $\widehat{\nabla}_{\mu}$ to get a fully covariant expression:

$$
\begin{aligned}
(4 \pi)^{d / 2} \mathscr{R}_{2}= & +\frac{1}{6} \Re X_{d / 2,1}[u]+X_{d / 2,1}[q]-\frac{1}{2}(d+2) g^{\mu v} X_{d / 2+1,2}\left[u \otimes \widehat{\nabla}_{\mu \nu}^{2} u\right] \\
& +(d+2) g^{\mu v} X_{d / 2+2,3}\left[u \otimes u \otimes \widehat{\nabla}_{\mu \nu}^{2} u\right]-X_{d / 2+1,2}\left[u \otimes \widehat{\nabla}_{\mu} p^{\mu}\right]-\frac{1}{2}(d+1) g^{\mu v} X_{d / 2+1,2}\left[\widehat{\nabla}_{\mu} u \otimes \widehat{\nabla}_{v} u\right] \\
& +\frac{1}{2}(d+2)(d+4) g^{\mu v} X_{d / 2+2,3}\left[u \otimes \widehat{\nabla}_{\mu} u \otimes \widehat{\nabla}_{\nu} u\right]+\frac{1}{2}(d+2) g^{\mu v} X_{d / 2+2,3}\left[\widehat{\nabla}_{\mu} u \otimes u \otimes \widehat{\nabla}_{\nu} u\right] \\
& -(d+2)(d+4) g^{\mu v} X_{d / 2+3,4}\left[u \otimes u \otimes \widehat{\nabla}_{\mu} u \otimes \widehat{\nabla}_{\nu} u\right]-\frac{1}{2}(d+2)(d+4) g^{\mu v} X_{d / 2+3,4}\left[u \otimes \widehat{\nabla}_{\mu} u \otimes u \otimes \widehat{\nabla}_{\nu} u\right] \\
& -\frac{1}{2}(d+1) X_{d / 2+1,2}\left[p^{\mu} \otimes \widehat{\nabla}_{\mu} u\right]+\frac{1}{2}(d+2) X_{d / 2+2,3}\left[u \otimes p^{\mu} \otimes \widehat{\nabla}_{\mu} u\right]+\frac{1}{2}(d+2) X_{d / 2+2,3}\left[p^{\mu} \otimes u \otimes \widehat{\nabla}_{\mu} u\right] \\
& -\frac{1}{2} X_{d / 2+1,2}\left[\widehat{\nabla}_{\mu} u \otimes p^{\mu}\right]+\frac{1}{2}(d+2) X_{d / 2+2,3}\left[u \otimes \widehat{\nabla}_{\mu} u \otimes p^{\mu}\right]-\frac{1}{2} g_{\mu \nu} X_{d / 2+1,2}\left[p^{\mu} \otimes p^{v}\right] .
\end{aligned}
$$


In this expression the contribution of a few terms can be simplified using Lemma 2.5 like:

$$
\begin{aligned}
& -\frac{1}{2}(d+2) g^{\mu v} X_{d / 2+1,2}\left[u \otimes \widehat{\nabla}_{\mu \nu}^{2} u\right]+(d+2) g^{\mu v} X_{d / 2+2,3}\left[u \otimes u \otimes \widehat{\nabla}_{\mu \nu}^{2} u\right]=-\frac{1}{2}(d+2) g^{\mu v} X_{d / 2+2,3}\left[u \otimes \widehat{\nabla}_{\mu \nu}^{2} u \otimes u\right], \\
& +\frac{1}{2}(d+2)(d+4) g^{\mu v} X_{d / 2+2,3}\left[u \otimes \widehat{\nabla}_{\mu} u \otimes \widehat{\nabla}_{\nu} u\right]-(d+2)(d+4) g^{\mu v} X_{d / 2+3,4}\left[u \otimes u \otimes \widehat{\nabla}_{\mu} u \otimes \widehat{\nabla}_{\nu} u\right] \\
& -\frac{1}{2}(d+2)(d+4) g^{\mu v} X_{d / 2+3,4}\left[u \otimes \widehat{\nabla}_{\mu} u \otimes u \otimes \widehat{\nabla}_{\nu} u\right] \\
& =\frac{1}{2}(d+2)(d+4) g^{\mu v} X_{d / 2+3,4}\left[u \otimes \widehat{\nabla}_{\mu} u \otimes \widehat{\nabla}_{\nu} u \otimes u\right], \\
& -\frac{1}{2}(d+1) X_{d / 2+1,2}\left[p^{\mu} \otimes \widehat{\nabla}_{\mu} u\right]+\frac{1}{2}(d+2) X_{d / 2+2,3}\left[u \otimes p^{\mu} \otimes \widehat{\nabla}_{\mu} u\right]+\frac{1}{2}(d+2) X_{d / 2+2,3}\left[p^{\mu} \otimes u \otimes \widehat{\nabla}_{\mu} u\right] \\
& =\frac{1}{2} X_{d / 2+1,2}\left[p^{\mu} \otimes \widehat{\nabla}_{\mu} u\right]-\frac{1}{2}(d+2) X_{d / 2+2,3}\left[p^{\mu} \otimes \widehat{\nabla}_{\mu} u \otimes u\right] .
\end{aligned}
$$

Finally, we get:

Theorem 5.1 The section $\mathscr{R}_{2}$ of $\operatorname{End}(V)$ is

$$
\begin{aligned}
(4 \pi)^{d / 2} \mathscr{R}_{2}= & +\frac{1}{6} \Re X_{d / 2,1}[u]+X_{d / 2,1}[q]-\frac{1}{2}(d+2) g^{\mu v} X_{d / 2+2,3}\left[u \otimes \widehat{\nabla}_{\mu \nu}^{2} u \otimes u\right] \\
& -\frac{1}{2}(d+1) g^{\mu v} X_{d / 2+1,2}\left[\widehat{\nabla}_{\mu} u \otimes \widehat{\nabla}_{\nu} u\right]+\frac{1}{2}(d+2) g^{\mu v} X_{d / 2+2,3}\left[\widehat{\nabla}_{\mu} u \otimes u \otimes \widehat{\nabla}_{\nu} u\right] \\
& +\frac{1}{2}(d+2)(d+4) g^{\mu v} X_{d / 2+3,4}\left[u \otimes \widehat{\nabla}_{\mu} u \otimes \widehat{\nabla}_{\nu} u \otimes u\right]+\frac{1}{2} X_{d / 2+1,2}\left[p^{\mu} \otimes \widehat{\nabla}_{\mu} u\right]-X_{d / 2+1,2}\left[u \otimes \widehat{\nabla}_{\mu} p^{\mu}\right] \\
& -\frac{1}{2}(d+2) X_{d / 2+2,3}\left[p^{\mu} \otimes \widehat{\nabla}_{\mu} u \otimes u\right]-\frac{1}{2} X_{d / 2+1,2}\left[\widehat{\nabla}_{\mu} u \otimes p^{\mu}\right]+\frac{1}{2}(d+2) X_{d / 2+2,3}\left[u \otimes \widehat{\nabla}_{\mu} u \otimes p^{\mu}\right] \\
& -\frac{1}{2} g_{\mu \nu} X_{d / 2+1,2}\left[p^{\mu} \otimes p^{\nu}\right] .
\end{aligned}
$$

A lengthy computation shows that this is compatible with [14, Thm 2.4].

According to Lemma 2.5, the writing of (5.2) is not unique. For instance, using

$$
\begin{aligned}
& -\frac{1}{2}(d+1) g^{\mu v} X_{d / 2+1,2}\left[\widehat{\nabla}_{\mu} u \otimes \widehat{\nabla}_{\nu} u\right]+\frac{1}{2}(d+2) g^{\mu v} X_{d / 2+2,3}\left[\widehat{\nabla}_{\mu} u \otimes u \otimes \widehat{\nabla}_{\nu} u\right] \\
& \quad=\frac{1}{2} g^{\mu v} X_{d / 2+1,2}\left[\widehat{\nabla}_{\mu} u \otimes \widehat{\nabla}_{\nu} u\right]-\frac{1}{2}(d+2) g^{\mu v} X_{d / 2+2,3}\left[u \otimes \widehat{\nabla}_{\mu} u \otimes \widehat{\nabla}_{\nu} u\right]-\frac{1}{2}(d+2) g^{\mu v} X_{d / 2+2,3}\left[\widehat{\nabla}_{\mu} u \otimes \widehat{\nabla}_{\nu} u \otimes u\right],
\end{aligned}
$$

this expression can be factorized as:

\section{Corollary 5.2}

$$
\begin{aligned}
(4 \pi)^{d / 2} \mathscr{R}_{2}= & +\frac{1}{6} \mathfrak{R} X_{d / 2,1}[u]+X_{d / 2,1}[q]-X_{d / 2+1,2}\left[u \otimes \widehat{\nabla}_{\mu} p^{\mu}\right]+\frac{1}{2} g^{\mu v} X_{d / 2+1,2}\left[\left(\widehat{\nabla}_{\mu} u+p_{\mu}\right) \otimes\left(\widehat{\nabla}_{\nu} u-p_{v}\right)\right] \\
& -\frac{1}{2}(d+2) g^{\mu v} X_{d / 2+2,3}\left[u \otimes \widehat{\nabla}_{\mu \nu}^{2} u \otimes u\right]-\frac{1}{2}(d+2) g^{\mu v} X_{d / 2+2,3}\left[u \otimes \widehat{\nabla}_{\mu} u \otimes\left(\widehat{\nabla}_{\nu} u-p_{v}\right)\right] \\
& -\frac{1}{2}(d+2) g^{\mu v} X_{d / 2+2,3}\left[\left(\widehat{\nabla}_{\mu} u+p_{\mu}\right) \otimes \widehat{\nabla}_{v} u \otimes u\right]+\frac{1}{2}(d+2)(d+4) g^{\mu v} X_{d / 2+3,4}\left[u \otimes \widehat{\nabla}_{\mu} u \otimes \widehat{\nabla}_{\nu} u \otimes u\right] .
\end{aligned}
$$

We do not know if this proposed factorization has some structural origin.

As explained just after Lemma 3.1, it can be useful to rewrite this result in terms of $N^{v}$ :

\section{Corollary 5.3}

$$
\begin{aligned}
(4 \pi)^{d / 2} \mathscr{R}_{2}= & +\frac{1}{6} \mathfrak{R} X_{d / 2,1}[u]+X_{d / 2,1}[q]+g^{\mu v} X_{d / 2+1,2}\left[u \otimes\left(\widehat{\nabla}_{\mu \nu}^{2} u\right)\right]-\frac{1}{2}(d+2) g^{\mu v} X_{d / 2+2,3}\left[u \otimes\left(\widehat{\nabla}_{\mu \nu}^{2} u\right) \otimes u\right] \\
& \left.-(d+2) g^{\mu v} X_{d / 2+2,3}\left[u \otimes\left(\widehat{\nabla}_{\mu} u\right) \otimes\left(\widehat{\nabla}_{v} u\right)\right]+\frac{1}{2}(d+2)(d+4) g^{\mu v} X_{d / 2+3,4}\left[u \otimes \widehat{\nabla}_{\mu} u\right) \otimes\left(\widehat{\nabla}_{v} u\right) \otimes u\right] \\
& +X_{d / 2+1,2}\left[N^{\mu} \otimes\left(\widehat{\nabla}_{\mu} u\right)\right]-\frac{1}{2}(d+2) X_{d / 2+2,3}\left[N^{\mu} \otimes\left(\widehat{\nabla}_{\mu} u\right) \otimes u\right]+\frac{1}{2}(d+2) X_{d / 2+2,3}\left[u \otimes\left(\widehat{\nabla}_{\mu} u\right) \otimes N^{\mu}\right] \\
& -\frac{1}{2} g_{\mu \nu} X_{d / 2+1,2}\left[N^{\mu} \otimes N^{v}\right]-X_{d / 2+1,2}\left[u \otimes\left(\widehat{\nabla}_{\mu} N^{\mu}\right)\right] .
\end{aligned}
$$

\section{The code}

The computation of $\mathscr{R}_{2}$ exposed in Section 5 shows that the simplified method summarized in Proposition 4.3 consists to apply a set of (mainly algebraic) rules at the level of the arguments of operators. This is to be contrasted with other methods based on more analytical properties of the heat coefficients, where for instance all possible expressions (based on the theory of invariants) are given by hand and their respective weights are computed (see [10] for instance). While these latter methods cannot be easily managed with a computer, the present method, being algebraic, can be translated into an algorithm. 
The first step in the computation of $\mathscr{R}_{2}$ makes appear a collection of fewer than 30 terms: they can be managed by hand. However, the same part of the computation for $\mathscr{R}_{4}$ produces thousands of terms. This is why a computer is needed to perform this computation.

Let us describe in this non-technical section the main characteristics of the computer code elaborated to make possible this computation.

Some computer algebraic systems (CAS) have been evaluated as a possible basis for this code. But, to our best knowledge, none of them was able to manage, in a easy way and without adding external modules, all the complexity of this computation. Indeed, formal manipulations have to be performed, to list a few, on commutative and noncommutative objects, on derivations $\left(\nabla_{\mu}, \widehat{\nabla}_{\mu}, \partial_{\mu}\right)$, on Riemannian structures (metric, Christoffel symbols, Ricci and Riemann tensors...), on contraction of tensors, on gauge structures $\left(A_{\mu}\right.$ and its curvature $F_{\mu \nu}$ ), on tensor products, on polynomials (in the dimension parameter $d$ )... Starting a formal computational code from the very beginning, as we did, has the following two main advantages: its purpose is to manipulate the necessary structures encountered in the computation, and only these structures; its internal model is based on the "mathematical model" that the method reveals.

This last point is a strong motivation to use an object oriented language in order to internally reproduce and manipulate, in a "natural" way, all the mathematical structures describing the key ingredient in which the method (and so the code) focuses: the "arguments" of the operators $X_{\alpha, k}$, as explained in Proposition 4.3. So, the code is built from the beginning on objects such as polynomials, commutative and noncommutative "elements" (for instance Riemannian tensors or matrixvalued functions), derivations (which can be applied, in a repetitive way, on the previously mentioned elements), products of elements (respecting commutative and noncommutative rules), tensor products, and finally the "arguments" of the $X_{\alpha, k}$ operators with collected commutative elements in front and the presence of the $Q[A]$ matrix-valued polynomials "on the right".

The object oriented language selected is JavaScript, the powerful language used in web browsers. This choice relies on various motivations. One of us was familiar with this language, and this helped to produce an efficient code quickly. The Node runtime ${ }^{1}$ permits to execute JavaScript as a scripting language in a terminal and it makes possible to read and write files. ${ }^{2}$ Moreover, the execution relies on the open source version of the very optimized JavaScript engine V8: ${ }^{3}$ benchmarks are very favorably compared to Python for instance (a language that would have been a good choice also). All the results are saved in files using the (open and native) format $\mathrm{JSON}^{4}$ and these results can be read as inputs for further computations. The translation of the code could be done into any other modern object oriented language.

On top of the main objects that the code can manipulate (with "natural methods" from a mathematical point of view), specific (higher level) functions have been coded to reproduce mathematical rules, like for instance contractions of Riemannian tensors, raising of indices, some simplifications... Substitutions of "elements" by more complicated structures are also made possible: these permits to reproduce the steps described in Section 5, where the computation is first done on the mathematical objects $H^{\mu \nu}, L^{\mu}$, and $q$ and then, in a second step, these objects (and their derivatives) are substituted using rules given in Section 3.2 (into normal coordinates). Substitutions rules can be hard coded or computed.

One of the main challenges when constructing such a code is to be able to simplify expressions to collect similar terms. This has required quite a lot of work to construct a "normalized" internal representation of terms (taking into account ambiguities on commutativity of elements, ambiguities on labeling indices in tensor contractions...). In that respect, the code may not compare to more mature CAS. A way to bypass this weakness was to make the exportation possible to inject expressions into another CAS: Our choice was to use Mathematica to perform formal computations (mainly to simplify the results at the very end of the computation) and to inject back the obtained results in the code.

The code can also export the generated expressions in $\mathrm{HT}_{\mathrm{E}} \mathrm{X}$, and all the results presented in this paper are those directly obtained in this way. Only the final layout has been adapted to reduce space.

Let us now explain the main steps of the computation of $\mathscr{R}_{4}$. One of the main ingredients in the computation is the substitution rule described in Section 3.2. In order to avoid as much as possible any transcription errors, the choice was made to only hard code the substitutions (3.13) and (3.14) for the metric and its derivatives (up to four) to normal coordinates. So, a preliminary step consists in computing (and save for later use) all the necessary substitutions of covariant derivatives of $H^{\mu \nu}, L^{\mu}$, and $q$ (up to the necessary number of derivations for the computation of $\mathscr{R}_{4}$ ) in terms of Riemannian tensors and derivations of $u, N^{\mu}$ and $q$. The subsequent preliminary step is to compute the replacement of the covariant derivative $\nabla_{\mu}$ by the total covariant derivative $\widehat{\nabla}_{\mu}$ (up to the necessary number of derivations) on these latter elements.

Then, after these preliminary results are stored, the main computation starts with the propagation of covariant derivations as given by the rule (4.12). Terms are next collected according to $Q[A]$ in order to apply, sequentially to these reduced numbers of terms, the following steps:

\footnotetext{
${ }^{1}$ https://nodejs.org

${ }^{2}$ Since it uses an extension of the "strict" JavaScript language used in web browsers.

${ }^{3}$ On which a lot of software engineers are working in a big private company...

4 "JavaScript Object Notation", a very convenient human readable structure format for data, that any modern language can read and write.
} 
- Substitutions are performed to generate expressions in normal coordinates.

- The necessary contractions with the tensors $G_{\mu_{1} \ldots \mu_{2 p}}$ (computed on the fly) are performed.

- A series of rules (contractions of tensors, raising of indices...) is applied to all the terms as long as these rules can be applied.

- The "expansion" rules of Lemma 2.5 are applied to leverage terms with same patterns ${ }^{5}$ to a common number $k$ of arguments, so that they can be compared.

- A full simplification of the obtained sum is performed by adding similar terms.

- The results are then saved in JSON file format for later use and in $\mathrm{HT}_{\mathrm{E}} \mathrm{X}$ for human reading.

Terms with same patterns are then collected in partial sums since they can produce simpler expressions, thanks to the use of Lemma 2.5, this time to reduce, as much as possible, the number of arguments $k$ and the number of terms. There is no uniqueness in this simplification procedure and a balance has to be found to produce these simplified expressions. This process has been mainly done in Mathematica after exportation of these partial sums. Once simplified expressions are obtained, they are written (by hand) in files that the code uses as inputs to compare them to their original (non simplified) versions. This series of checks, the last step of the computation, also exports the simplified expressions in $\mathrm{ET}_{\mathrm{E}} \mathrm{X}$ : they are the expressions presented in this paper.

The code produces new results for $\mathscr{R}_{4}$ which, to our best knowledge, never appeared before. So, some comparisons against known results have to be performed to confirm the validity of the code (at least partially). Three tests have been successful:

1. The computation of $\mathscr{R}_{2}$ reproduces the result obtained by hand (this is the result given in Corollary 5.3).

2. The computation of $\mathscr{R}_{2}$ for the 2-dimensional noncommutative torus produces results in agreement with those in [14] (once translated back into spectral functions): the interested reader will find this result in $\mathrm{BT}_{\mathrm{E}} \mathrm{X}$ files accompanying the open source code [15].

3. The case $u$ parallel (Section 7.3) is a special case for $\mathscr{R}_{4}$ in which all the derivations of $u$ are put to zero. With the further specifications $u=\mathbb{1}$ and $N^{\mu}=0$, the result agrees with [12, Theorem 3.3.1].

Notice that the consistency of gauge invariant expressions in the $Q[A]$-part of the results (see Section 7.1) is also a strong requirement for the global validity of the code.

The code has been written with the method in mind, not for the computation of $\mathscr{R}_{4}$ in particular. This means that it can be used to compute $\mathscr{R}_{r}$ for $r \geq 6$ (and then the number of generated terms will be huge!) and it can also be appropriate in situations where some elements take specific values (for instance, in the 2-dimensional noncommutative torus case, all the elements are written in terms of a single positive element in the noncommutative algebra). This makes the code flexible enough for further computations of heat coefficients for non minimal Laplace type operators.

Anyone can contribute to the open-source code (GNU GPL v3 License) we produced [15] and can use it as a starting point for his/her projects as far as the required computations are accessible by the method exposed in this paper.

\section{Results on $\mathscr{R}_{4}$ produced by computer}

To compute $\mathscr{R}_{4}$ in $a_{4}(a, P)(x)=\operatorname{tr}\left[a(x) \mathscr{R}_{4}(x)\right]$, we start with

$$
\begin{aligned}
(4 \pi)^{d / 2} \mathscr{R}_{4}= & X_{d / 2,2}[P \otimes P]-X_{d / 2+1,3, \mu_{1} \mu_{2}}\left[K^{\mu_{1}} \otimes K^{\mu_{2}} \otimes P\right]-X_{d / 2+1,3, \mu_{1} \mu_{2}}\left[K^{\mu_{1}} \otimes P \otimes K^{\mu_{2}}\right] \\
& -X_{d / 2+1,3, \mu_{1} \mu_{2}}\left[P \otimes K^{\mu_{1}} \otimes K^{\mu_{2}}\right]+X_{d / 2+2,4, \mu_{1} \mu_{2} \mu_{3} \mu_{4}}\left[K^{\mu_{1}} \otimes K^{\mu_{2}} \otimes K^{\mu_{3}} \otimes K^{\mu_{4}}\right] .
\end{aligned}
$$

Here, the series of spectral operators $X_{\alpha, k}$ appearing in the computation are $X_{d / 2-1,1}, X_{d / 2,2}, X_{d / 2+1,3}, \ldots, X_{d / 2+k-2, k}$ for $k=1, \ldots, 6$. Thus, in this section we adopt the shorthands $X_{k}$ for $k=1, \ldots, 6$.

Application of (4.12) is done using a computer because it gives too many terms. Actually, after simplification, it produces thousands of terms, that can be sorted according to only 5 values of $Q[A] v=\nabla^{k} v$ produced for $v \in \mathbb{C}^{N}: v, \nabla_{\nu_{1}} v$, $\nabla_{v_{1} v_{2}}^{2} \nu, \nabla_{v_{1} v_{2} v_{3}}^{3} v$, and $\nabla_{v_{1} v_{2} v_{3} v_{4}}^{4} v$. These five sums are denoted $\mathscr{R}_{4, k}$ for $k \in\{0,1,2,3,4\}$, thus

$$
\mathscr{R}_{4}(x)=\sum_{k=0}^{4} \mathscr{R}_{4, k}(x)
$$

Since the factors in front of $Q[A]$ have homogeneous gauge transformations, the result should be written as (explicitly) gauge homogeneous expressions to ensure that $\mathscr{R}_{4}(x)$ transforms homogeneously.

\footnotetext{
${ }^{5}$ A "pattern" of an argument is the reduced ordered list of elements appearing in this argument (forgetting the polynomials in dimension $d$ in front of it) where the $u$ elements (without applied derivation) are omitted.
} 
To simplify, the results are presented with $N^{\mu}=0$, but the interested reader will find the general case in $\mathrm{BT}_{\mathrm{E} X} \mathrm{X}$ files accompanying the open source code [15].

\subsection{Computation of $\mathscr{R}_{4, k}$ for $k \neq 0$}

When $Q[A] v=\nabla_{v_{1} v_{2} v_{3} v_{4}}^{4} v$, the factor can be computed by hand from the very beginning because only few terms contribute. The only possible gauge homogeneous expression is $F^{v_{1} v_{2}} F_{v_{1} v_{2}}$ and indeed the computer returns directly only one term:

$$
(4 \pi)^{d / 2} \mathscr{R}_{4,4}=+\frac{1}{12} X_{1}[u] F^{v_{1} v_{2}} F_{v_{1} v_{2}} .
$$

For $Q[A] v=\nabla_{v_{1} v_{2} v_{3}}^{3} v$, the computer produces terms that can be sorted following a repeating pattern:

$$
\left(g^{\nu_{1} v_{2}} b^{\nu_{3}}-2 g^{\nu_{1} v_{3}} b^{v_{2}}+g^{\nu_{2} v_{3}} b^{\nu_{1}}\right)\left(\nabla_{v_{1} \nu_{2} \nu_{3}}^{3} v\right) \text {. }
$$

In this expression, changing the summation variables and using the symmetry of metric indices, the gauge homogeneous expression $\nabla_{v_{1}} F_{v_{2} v_{3}}$ appears automatically:

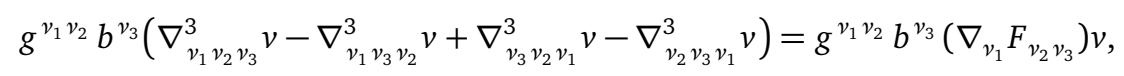

because using (3.6),

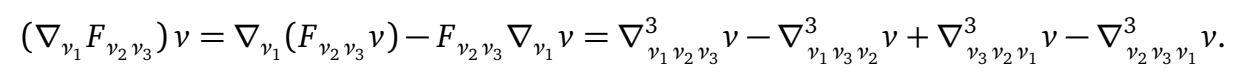

More precisely, this gives, since $\nabla_{v_{1}} F_{v_{2} v_{3}} \stackrel{\text { n.c. }}{\longrightarrow} \widehat{\nabla}_{v_{1}} F_{v_{2} v_{3}}$,

$$
\begin{aligned}
(4 \pi)^{d / 2} \mathscr{R}_{4,3}= & +\frac{1}{6}(d-2) g^{v_{1} v_{2}} g^{v_{3} v_{4}} X_{2}\left[u \otimes\left(\widehat{\nabla}_{v_{4}} u\right)\right]\left(\widehat{\nabla}_{v_{1}} F_{v_{2} v_{3}}\right)-d g^{v_{1} v_{2}} g^{v_{3} v_{4}} X_{3}\left[u \otimes u \otimes\left(\widehat{\nabla}_{v_{4}} u\right)\right]\left(\widehat{\nabla}_{v_{1}} F_{v_{2} v_{3}}\right) \\
& +2(d+2) g^{v_{1} v_{2}} g^{v_{3} v_{4}} X_{4}\left[u \otimes u \otimes u \otimes\left(\widehat{\nabla}_{v_{4}} u\right)\right]\left(\widehat{\nabla}_{v_{1}} F_{v_{2} v_{3}}\right) .
\end{aligned}
$$

When $Q[A] v=\nabla_{v_{1} v_{2}}^{2} v$, the symmetric part with respect to $v_{1}, v_{2}$ does not produce a gauge homogeneous term, thus must be zero. This is checked by the computer which only returns the skew symmetric part (the $F_{v_{1} v_{2}}$ tensor):

$$
\begin{aligned}
(4 \pi)^{d / 2} & \mathscr{R}_{4,2}= \\
& -d X_{3}\left[u \otimes\left(\widehat{\nabla}_{v_{1}} u\right) \otimes\left(\widehat{\nabla}_{v_{2}} u\right)\right] F^{v_{1} v_{2}}-4 X_{4}\left[u \otimes u \otimes\left(\widehat{\nabla}_{v_{1}} u\right) \otimes\left(\widehat{\nabla}_{v_{2}} u\right)\right] F^{v_{1} v_{2}} \\
& +\frac{1}{2}(d+2)(d+4) X_{5}\left[u \otimes\left(\widehat{\nabla}_{v_{1}} u\right) \otimes u \otimes\left(\widehat{\nabla}_{v_{2}} u\right) \otimes u\right] F^{v_{1} v_{2}}+2(d+4) X_{5}\left[u \otimes u \otimes\left(\widehat{\nabla}_{v_{1}} u\right) \otimes\left(\widehat{\nabla}_{v_{2}} u\right) \otimes u\right] F^{v_{1} v_{2}} \\
& +4(d+4) X_{5}\left[u \otimes u \otimes u \otimes\left(\widehat{\nabla}_{v_{1}} u\right) \otimes\left(\widehat{\nabla}_{v_{2}} u\right)\right] F^{v_{1} v_{2}}+(d+4)(d+6) X_{6}\left[u \otimes u \otimes\left(\widehat{\nabla}_{v_{1}} u\right) \otimes u \otimes u \otimes\left(\widehat{\nabla}_{v_{2}} u\right)\right] F^{v_{1} v_{2}} \\
& +(d+4)(d+6) X_{6}\left[u \otimes u \otimes u \otimes\left(\widehat{\nabla}_{v_{1}} u\right) \otimes u \otimes\left(\widehat{\nabla}_{v_{2}} u\right)\right] F^{v_{1} v_{2}} \\
& \quad-\frac{1}{2} d X_{3}\left[u \otimes u \otimes\left[F_{v_{1} v_{2}}, u\right]\right] F^{v_{1} v_{2}}+(d+2) X_{4}\left[u \otimes u \otimes u \otimes\left[F_{v_{1} v_{2}}, u\right]\right] F^{v_{1} v_{2}} \\
& -(d+4) X_{5}\left[u \otimes u \otimes u \otimes u \otimes\left[F_{v_{1} v_{2}}, u\right]\right] F^{v_{1} v_{2}} .
\end{aligned}
$$

The contribution $\mathscr{R}_{4,1}$ with $Q[A] v=\nabla_{v_{1}} v$ does not transform homogeneously because $\nabla_{v_{1}} v=A_{v_{1}} v$, and one cannot produce a gauge homogeneous expression as a polynomial of $A_{v}$ of degree 1 with no derivations. As a consequence, $\mathscr{R}_{4,1}$ should vanish and this is what the computer returns:

$$
\mathscr{R}_{4,1}=0 .
$$

\subsection{Computation of $\mathscr{R}_{4,0}$}

Here we use the following notations: $G^{v_{1} v_{2} v_{3} v_{4}}:=\frac{1}{4}\left(g^{\nu_{1} \nu_{2}} g^{v_{3} v_{4}}+g^{v_{1} v_{3}} g^{v_{2} v_{4}}+g^{v_{1} v_{4}} g^{v_{2} \nu_{3}}\right)$ and

$$
\widehat{\Delta}_{\mu \nu}:=\frac{1}{2}\left(\widehat{\nabla}_{\mu} \widehat{\nabla}_{\nu}+\widehat{\nabla}_{\nu} \widehat{\nabla}_{\mu}\right), \quad\left\{\widehat{\Delta}, \widehat{\nabla}_{v}\right\}:=\widehat{\Delta} \widehat{\nabla}_{\nu}+\widehat{\nabla}_{\nu} \widehat{\Delta}
$$

so that $\widehat{\nabla}_{\mu \nu}^{2}=\widehat{\Delta}_{\mu \nu}+\frac{1}{2} F_{\mu \nu}$ and $\widehat{\Delta}=g^{\mu \nu} \widehat{\nabla}_{\mu \nu}^{2}=g^{\mu \nu} \widehat{\Delta}_{\mu \nu}$. 
The computer produces around 400 terms for $\mathscr{R}_{4,0}$, but after simplifications based on (2.24), this reduces to the following 180 terms collected according to their pattern:

$$
\begin{aligned}
& (4 \pi)^{d / 2} \mathscr{R}_{4,0}= \\
& +\frac{1}{180}|R|^{2} X_{1}[u]-\frac{1}{180}|\operatorname{Ric}|^{2} X_{1}[u]+\frac{1}{30}(\widehat{\Delta} \mathfrak{R}) X_{1}[u]+\frac{1}{72} \mathfrak{R}^{2} X_{1}[u] \\
& -\frac{1}{12}(d-2) g^{v_{1} v_{2}}\left(\widehat{\nabla}_{v_{2}} \Re\right) X_{2}\left[u \otimes\left(\widehat{\nabla}_{\nu_{1}} u\right)\right]+\frac{1}{2}(d+2) g^{v_{1} v_{2}}\left(\widehat{\nabla}_{v_{2}} \Re\right) X_{4}\left[u \otimes u \otimes u \otimes\left(\widehat{\nabla}_{v_{1}} u\right)\right] \\
& -\frac{1}{3} d \operatorname{Ric}^{v_{1} v_{2}} X_{3}\left[u \otimes\left(\widehat{\nabla}_{v_{1}} u\right) \otimes\left(\widehat{\nabla}_{v_{2}} u\right)\right]+\frac{1}{3}(d+2)(d+3) \operatorname{Ric}^{\nu_{1} v_{2}} X_{4}\left[u \otimes u \otimes\left(\widehat{\nabla}_{v_{1}} u\right) \otimes\left(\widehat{\nabla}_{v_{2}} u\right)\right] \\
& -\frac{1}{12} d(d+2) \operatorname{Ric}^{v_{1} v_{2}} X_{4}\left[u \otimes\left(\widehat{\nabla}_{v_{1}} u\right) \otimes u \otimes\left(\widehat{\nabla}_{v_{2}} u\right)\right]-(d+2)(d+4) \operatorname{Ric}^{\nu_{1} v_{2}} X_{5}\left[u \otimes u \otimes u \otimes\left(\widehat{\nabla}_{v_{1}} u\right) \otimes\left(\widehat{\nabla}_{v_{2}} u\right)\right] \\
& +\frac{1}{4}(d+2)(d+4) \operatorname{Ric}^{v_{1} v_{2}} X_{5}\left[u \otimes\left(\widehat{\nabla}_{v_{1}} u\right) \otimes u \otimes\left(\widehat{\nabla}_{v_{2}} u\right) \otimes u\right] \\
& -\frac{1}{6} d g^{v_{1} v_{2}} \Re X_{3}\left[u \otimes\left(\widehat{\nabla}_{v_{1}} u\right) \otimes\left(\widehat{\nabla}_{v_{2}} u\right)\right]+\frac{1}{12} d(d+2) g^{v_{1} v_{2}} \Re X_{4}\left[u \otimes\left(\widehat{\nabla}_{v_{1}} u\right) \otimes\left(\widehat{\nabla}_{v_{2}} u\right) \otimes u\right] \\
& -2(d+4)\left(d^{2}+10 d+28\right) G^{v_{1} v_{2} v_{3} v_{4}} X_{5}\left[u \otimes\left(\widehat{\nabla}_{v_{1}} u\right) \otimes\left(\widehat{\nabla}_{v_{2}} u\right) \otimes\left(\widehat{\nabla}_{v_{3}} u\right) \otimes\left(\widehat{\nabla}_{v_{4}} u\right)\right] \\
& +4(d+4)(d+6) G^{v_{1} v_{2} v_{3} v_{4}} X_{6}\left[u \otimes\left(\widehat{\nabla}_{v_{1}} u\right) \otimes\left(\widehat{\nabla}_{v_{2}} u\right) \otimes\left(\widehat{\nabla}_{v_{3}} u\right) \otimes\left(\widehat{\nabla}_{v_{4}} u\right) \otimes u\right] \\
& +2(d+4)(d+6)^{2} G^{v_{1} v_{2} v_{3} v_{4}} X_{6}\left[u \otimes\left(\widehat{\nabla}_{v_{1}} u\right) \otimes\left(\widehat{\nabla}_{v_{2}} u\right) \otimes\left(\widehat{\nabla}_{v_{3}} u\right) \otimes u \otimes\left(\widehat{\nabla}_{v_{4}} u\right)\right] \\
& +2(d+4)^{2}(d+6) G^{v_{1} v_{2} v_{3} v_{4}} X_{6}\left[u \otimes\left(\widehat{\nabla}_{v_{1}} u\right) \otimes\left(\widehat{\nabla}_{v_{2}} u\right) \otimes u \otimes\left(\widehat{\nabla}_{v_{3}} u\right) \otimes\left(\widehat{\nabla}_{v_{4}} u\right)\right] \\
& -4(d+4)\left(d^{2}+8 d+28\right) G^{v_{1} v_{2} v_{3} v_{4}} X_{6}\left[u \otimes u \otimes\left(\widehat{\nabla}_{v_{1}} u\right) \otimes\left(\widehat{\nabla}_{v_{2}} u\right) \otimes\left(\widehat{\nabla}_{v_{3}} u\right) \otimes\left(\widehat{\nabla}_{v_{4}} u\right)\right] \\
& -2(d+2)(d+4)(d+6) G^{v_{1} v_{2} v_{3} v_{4}} X_{6}\left[u \otimes\left(\widehat{\nabla}_{v_{1}} u\right) \otimes u \otimes\left(\widehat{\nabla}_{v_{2}} u\right) \otimes\left(\widehat{\nabla}_{v_{3}} u\right) \otimes\left(\widehat{\nabla}_{v_{4}} u\right)\right] \\
& +8(d+4)\left(d^{2}+10 d+32\right) G^{v_{1} v_{2} v_{3} v_{4}} X_{7}\left[u \otimes u \otimes\left(\widehat{\nabla}_{v_{1}} u\right) \otimes\left(\widehat{\nabla}_{v_{2}} u\right) \otimes u \otimes\left(\widehat{\nabla}_{v_{3}} u\right) \otimes\left(\widehat{\nabla}_{v_{4}} u\right)\right] \\
& +4(d+4)\left(d^{2}+6 d+16\right) G^{v_{1} v_{2} v_{3} v_{4}} X_{7}\left[u \otimes u \otimes\left(\widehat{\nabla}_{v_{1}} u\right) \otimes\left(\widehat{\nabla}_{v_{2}} u\right) \otimes\left(\widehat{\nabla}_{v_{3}} u\right) \otimes u \otimes\left(\widehat{\nabla}_{v_{4}} u\right)\right]
\end{aligned}
$$

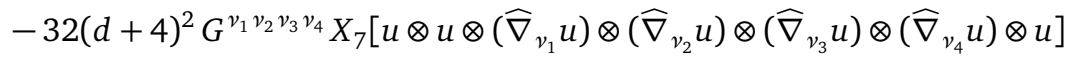

$$
\begin{aligned}
& +8(d+4)^{2}(d+6) G^{v_{1} v_{2} v_{3} v_{4}} X_{7}\left[u \otimes\left(\widehat{\nabla}_{v_{1}} u\right) \otimes u \otimes u \otimes\left(\widehat{\nabla}_{v_{2}} u\right) \otimes\left(\widehat{\nabla}_{v_{3}} u\right) \otimes\left(\widehat{\nabla}_{v_{4}} u\right)\right] \\
& +4(d+4)^{2}(d+6) G^{v_{1} v_{2} v_{3} v_{4}} X_{7}\left[u \otimes\left(\widehat{\nabla}_{v_{1}} u\right) \otimes u \otimes\left(\widehat{\nabla}_{v_{2}} u\right) \otimes u \otimes\left(\widehat{\nabla}_{v_{3}} u\right) \otimes\left(\widehat{\nabla}_{v_{4}} u\right)\right] \\
& +2 d(d+4)(d+6) G^{v_{1} v_{2} v_{3} v_{4}} X_{7}\left[u \otimes\left(\widehat{\nabla}_{v_{1}} u\right) \otimes u \otimes\left(\widehat{\nabla}_{v_{2}} u\right) \otimes\left(\widehat{\nabla}_{v_{3}} u\right) \otimes u \otimes\left(\widehat{\nabla}_{v_{4}} u\right)\right] \\
& -16(d+4)(d+6) G^{v_{1} v_{2} v_{3} v_{4}} X_{7}\left[u \otimes\left(\widehat{\nabla}_{v_{1}} u\right) \otimes u \otimes\left(\widehat{\nabla}_{v_{2}} u\right) \otimes\left(\widehat{\nabla}_{v_{3}} u\right) \otimes\left(\widehat{\nabla}_{v_{4}} u\right) \otimes u\right] \\
& +2(d+4)(d+6)(d+8) G^{v_{1} v_{2} v_{3} v_{4}} X_{7}\left[u \otimes\left(\widehat{\nabla}_{v_{1}} u\right) \otimes\left(\widehat{\nabla}_{v_{2}} u\right) \otimes u \otimes\left(\widehat{\nabla}_{v_{3}} u\right) \otimes\left(\widehat{\nabla}_{v_{4}} u\right) \otimes u\right] \\
& +24(d+4)\left(d^{2}+10 d+32\right) G^{v_{1} v_{2} v_{3} v_{4}} X_{7}\left[u \otimes u \otimes u \otimes\left(\widehat{\nabla}_{v_{1}} u\right) \otimes\left(\widehat{\nabla}_{v_{2}} u\right) \otimes\left(\widehat{\nabla}_{v_{3}} u\right) \otimes\left(\widehat{\nabla}_{v_{4}} u\right)\right] \\
& +16(d+4)\left(d^{2}+10 d+28\right) G^{v_{1} v_{2} v_{3} v_{4}} X_{7}\left[u \otimes u \otimes\left(\widehat{\nabla}_{v_{1}} u\right) \otimes u \otimes\left(\widehat{\nabla}_{v_{2}} u\right) \otimes\left(\widehat{\nabla}_{v_{3}} u\right) \otimes\left(\widehat{\nabla}_{v_{4}} u\right)\right] \\
& +4(d+4)(d+6)(d+8)(d+10) G^{v_{1} v_{2} v_{3} v_{4}} X_{8}\left[u \otimes u \otimes\left(\widehat{\nabla}_{v_{1}} u\right) \otimes\left(\widehat{\nabla}_{v_{2}} u\right) \otimes\left(\widehat{\nabla}_{v_{3}} u\right) \otimes\left(\widehat{\nabla}_{v_{4}} u\right) \otimes u \otimes u\right] \\
& +2(d+4)(d+6)(d+8)(d+10) G^{v_{1} v_{2} v_{3} v_{4}} X_{8}\left[u \otimes\left(\widehat{\nabla}_{v_{1}} u\right) \otimes u \otimes\left(\widehat{\nabla}_{v_{2}} u\right) \otimes\left(\widehat{\nabla}_{v_{3}} u\right) \otimes\left(\widehat{\nabla}_{v_{4}} u\right) \otimes u \otimes u\right] \\
& +(d+4)(d+6)(d+8)(d+10) G^{v_{1} v_{2} v_{3} v_{4}} X_{8}\left[u \otimes\left(\widehat{\nabla}_{v_{1}} u\right) \otimes u \otimes\left(\widehat{\nabla}_{v_{2}} u\right) \otimes\left(\widehat{\nabla}_{v_{3}} u\right) \otimes u \otimes\left(\widehat{\nabla}_{v_{4}} u\right) \otimes u\right] \\
& +2(d+4)(d+6)(d+8)(d+10) G^{v_{1} v_{2} v_{3} v_{4}} X_{8}\left[u \otimes u \otimes\left(\widehat{\nabla}_{v_{1}} u\right) \otimes\left(\widehat{\nabla}_{v_{2}} u\right) \otimes\left(\widehat{\nabla}_{v_{3}} u\right) \otimes u \otimes\left(\widehat{\nabla}_{v_{4}} u\right) \otimes u\right] \\
& -\frac{1}{2}(d+2)(d+4)^{2} g^{v_{1} v_{2}} g^{v_{3} v_{4}} X_{5}\left[u \otimes\left(\widehat{\nabla}_{v_{1}} u\right) \otimes\left(\widehat{\nabla}_{v_{2}} u\right) \otimes\left(\widehat{\nabla}_{v_{3}} u\right) \otimes\left(\widehat{\nabla}_{v_{4}} u\right)\right] \\
& +\frac{1}{2}(d+2)(d+4)(d+6) g^{v_{1} v_{2}} g^{v_{3} v_{4}} X_{6}\left[u \otimes\left(\widehat{\nabla}_{v_{1}} u\right) \otimes\left(\widehat{\nabla}_{v_{2}} u\right) \otimes\left(\widehat{\nabla}_{v_{3}} u\right) \otimes u \otimes\left(\widehat{\nabla}_{v_{4}} u\right)\right] \\
& +\frac{1}{2}(d+2)(d+4)(d+6) g^{v_{1} v_{2}} g^{v_{3} v_{4}} X_{6}\left[u \otimes\left(\widehat{\nabla}_{v_{1}} u\right) \otimes u \otimes\left(\widehat{\nabla}_{v_{2}} u\right) \otimes\left(\widehat{\nabla}_{v_{3}} u\right) \otimes\left(\widehat{\nabla}_{v_{4}} u\right)\right] \\
& +(d+2)(d+4)(d+6) g^{v_{1} v_{2}} g^{v_{3} v_{4}} X_{6}\left[u \otimes u \otimes\left(\widehat{\nabla}_{v_{1}} u\right) \otimes\left(\widehat{\nabla}_{v_{2}} u\right) \otimes\left(\widehat{\nabla}_{v_{3}} u\right) \otimes\left(\widehat{\nabla}_{v_{4}} u\right)\right] \\
& +\frac{1}{4}(d+2)(d+4)(d+6)(d+8) g^{v_{1} v_{2}} g^{v_{3} v_{4}} X_{7}\left[u \otimes\left(\widehat{\nabla}_{v_{1}} u\right) \otimes\left(\widehat{\nabla}_{v_{2}} u\right) \otimes u \otimes\left(\widehat{\nabla}_{v_{3}} u\right) \otimes\left(\widehat{\nabla}_{v_{4}} u\right) \otimes u\right] \\
& -\frac{1}{12}(d-2) \Re X_{2}[u \otimes(\widehat{\Delta} u)]+\frac{1}{6} d \Re X_{3}[u \otimes u \otimes(\widehat{\Delta} u)] \\
& -\frac{1}{3} d \operatorname{Ric}^{v_{1} v_{2}} X_{3}\left[u \otimes u \otimes\left(\widehat{\Delta}_{v_{1} v_{2}} u\right)\right]+(d+2) \operatorname{Ric}^{v_{1} v_{2}} X_{4}\left[u \otimes u \otimes u \otimes\left(\widehat{\Delta}_{v_{1} v_{2}} u\right)\right] \\
& +\frac{1}{2}(d+2)^{2} g^{v_{1} v_{2}} X_{4}\left[u \otimes(\widehat{\Delta} u) \otimes\left(\widehat{\nabla}_{v_{1}} u\right) \otimes\left(\widehat{\nabla}_{v_{2}} u\right)\right]-\frac{1}{2}(d+2)(d+4) g^{v_{1} v_{2}} X_{5}\left[u \otimes(\widehat{\Delta} u) \otimes\left(\widehat{\nabla}_{v_{1}} u\right) \otimes u \otimes\left(\widehat{\nabla}_{v_{2}} u\right)\right] \\
& -(d+4)^{2} g^{v_{1} v_{2}} X_{5}\left[u \otimes u \otimes(\widehat{\Delta} u) \otimes\left(\widehat{\nabla}_{v_{1}} u\right) \otimes\left(\widehat{\nabla}_{v_{2}} u\right)\right]
\end{aligned}
$$


$-\frac{1}{4}(d+2)(d+4)(d+6) g^{v_{1} v_{2}} X_{6}\left[u \otimes(\widehat{\Delta} u) \otimes u \otimes\left(\widehat{\nabla}_{v_{1}} u\right) \otimes\left(\widehat{\nabla}_{v_{2}} u\right) \otimes u\right]$

$+(d+4)(d+6) g^{v_{1} v_{2}} X_{6}\left[u \otimes u \otimes(\widehat{\Delta} u) \otimes\left(\widehat{\nabla}_{v_{1}} u\right) \otimes u \otimes\left(\widehat{\nabla}_{v_{2}} u\right)\right]$

$-(d+2)(d+4)(d+6) g^{v_{1} v_{2}} X_{7}\left[u \otimes u \otimes(\widehat{\Delta} u) \otimes\left(\widehat{\nabla}_{v_{1}} u\right) \otimes\left(\widehat{\nabla}_{v_{2}} u\right) \otimes u \otimes u\right]$

$-\frac{1}{2}(d+2)(d+4)(d+6) g^{v_{1} v_{2}} X_{7}\left[u \otimes u \otimes(\widehat{\Delta} u) \otimes\left(\widehat{\nabla}_{v_{1}} u\right) \otimes u \otimes\left(\widehat{\nabla}_{v_{2}} u\right) \otimes u\right]$

$+3(d+4)(d+6) g^{v_{1} v_{2}} X_{7}\left[u \otimes u \otimes(\widehat{\Delta} u) \otimes u \otimes\left(\widehat{\nabla}_{v_{1}} u\right) \otimes\left(\widehat{\nabla}_{v_{2}} u\right) \otimes u\right]$

$+9(d+4)(d+6) g^{v_{1} v_{2}} X_{7}\left[u \otimes u \otimes u \otimes(\widehat{\Delta} u) \otimes\left(\widehat{\nabla}_{v_{1}} u\right) \otimes\left(\widehat{\nabla}_{v_{2}} u\right) \otimes u\right]$

$-(d+2) g^{v_{1} v_{2}} X_{4}\left[u \otimes\left(\widehat{\nabla}_{v_{1}} u\right) \otimes(\widehat{\Delta} u) \otimes\left(\widehat{\nabla}_{v_{2}} u\right)\right]+\frac{1}{2}(d+4)(3 d+14) g^{v_{1} v_{2}} X_{5}\left[u \otimes\left(\widehat{\nabla}_{v_{1}} u\right) \otimes(\widehat{\Delta} u) \otimes\left(\widehat{\nabla}_{v_{2}} u\right) \otimes u\right]$

$-2(d+4)(d+6) g^{v_{1} v_{2}} X_{6}\left[u \otimes\left(\widehat{\nabla}_{v_{1}} u\right) \otimes(\widehat{\Delta} u) \otimes\left(\widehat{\nabla}_{v_{2}} u\right) \otimes u \otimes u\right]$

$-(d+4)(d+6) g^{v_{1} v_{2}} X_{6}\left[u \otimes\left(\widehat{\nabla}_{v_{1}} u\right) \otimes(\widehat{\Delta} u) \otimes u \otimes\left(\widehat{\nabla}_{v_{2}} u\right) \otimes u\right]$

$+\frac{1}{2}(d+4)(d+6) g^{v_{1} v_{2}} X_{6}\left[u \otimes\left(\widehat{\nabla}_{v_{1}} u\right) \otimes u \otimes(\widehat{\Delta} u) \otimes u \otimes\left(\widehat{\nabla}_{v_{2}} u\right)\right]$

$+(d+4)(d+6) g^{v_{1} v_{2}} X_{6}\left[u \otimes u \otimes\left(\widehat{\nabla}_{v_{1}} u\right) \otimes(\widehat{\Delta} u) \otimes u \otimes\left(\widehat{\nabla}_{v_{2}} u\right)\right]$

$-\frac{1}{2}(d+4)(d+6)(d+8) g^{v_{1} v_{2}} X_{7}\left[u \otimes\left(\widehat{\nabla}_{v_{1}} u\right) \otimes u \otimes(\widehat{\Delta} u) \otimes\left(\widehat{\nabla}_{v_{2}} u\right) \otimes u \otimes u\right]$

$-\frac{1}{4}(d+4)(d+6)(d+8) g^{v_{1} v_{2}} X_{7}\left[u \otimes\left(\widehat{\nabla}_{v_{1}} u\right) \otimes u \otimes(\widehat{\Delta} u) \otimes u \otimes\left(\widehat{\nabla}_{v_{2}} u\right) \otimes u\right]$

$-(d+4)(d+6)(d+8) g^{v_{1} v_{2}} X_{7}\left[u \otimes u \otimes\left(\widehat{\nabla}_{v_{1}} u\right) \otimes(\widehat{\Delta} u) \otimes\left(\widehat{\nabla}_{v_{2}} u\right) \otimes u \otimes u\right]$

$-\frac{1}{2}(d+4)(d+6)(d+8) g^{v_{1} v_{2}} X_{7}\left[u \otimes u \otimes\left(\widehat{\nabla}_{v_{1}} u\right) \otimes(\widehat{\Delta} u) \otimes u \otimes\left(\widehat{\nabla}_{v_{2}} u\right) \otimes u\right]$

$-(d+2) g^{v_{1} v_{2}} X_{4}\left[u \otimes\left(\widehat{\nabla}_{v_{1}} u\right) \otimes\left(\widehat{\nabla}_{v_{2}} u\right) \otimes(\widehat{\Delta} u)\right]+\frac{1}{2}(d+4)(3 d+10) g^{v_{1} v_{2}} X_{5}\left[u \otimes\left(\widehat{\nabla}_{v_{1}} u\right) \otimes\left(\widehat{\nabla}_{v_{2}} u\right) \otimes(\widehat{\Delta} u) \otimes u\right]$

$+\frac{1}{2}(d+2)(d+4) g^{v_{1} v_{2}} X_{5}\left[u \otimes\left(\widehat{\nabla}_{v_{1}} u\right) \otimes\left(\widehat{\nabla}_{v_{2}} u\right) \otimes u \otimes(\widehat{\Delta} u)\right]$

$-(d+4)(d+6) g^{v_{1} v_{2}} X_{6}\left[u \otimes\left(\widehat{\nabla}_{v_{1}} u\right) \otimes\left(\widehat{\nabla}_{v_{2}} u\right) \otimes(\widehat{\Delta} u) \otimes u \otimes u\right]$

$+\frac{1}{2}(d+4)(d+6) g^{v_{1} v_{2}} X_{6}\left[u \otimes\left(\widehat{\nabla}_{v_{1}} u\right) \otimes\left(\widehat{\nabla}_{v_{2}} u\right) \otimes u \otimes(\widehat{\Delta} u) \otimes u\right]$

$-\frac{1}{2}(d+4)(d+6)(d+8) g^{v_{1} v_{2}} X_{7}\left[u \otimes\left(\widehat{\nabla}_{v_{1}} u\right) \otimes\left(\widehat{\nabla}_{v_{2}} u\right) \otimes u \otimes u \otimes(\widehat{\Delta} u) \otimes u\right]$

$-\frac{1}{2}(d+4)(d+6)(d+8) g^{v_{1} v_{2}} X_{7}\left[u \otimes\left(\widehat{\nabla}_{v_{1}} u\right) \otimes\left(\widehat{\nabla}_{v_{2}} u\right) \otimes u \otimes(\widehat{\Delta} u) \otimes u \otimes u\right]$

$-\frac{1}{2}(d+4)(d+6)(d+8) g^{v_{1} v_{2}} X_{7}\left[u \otimes\left(\widehat{\nabla}_{v_{1}} u\right) \otimes u \otimes\left(\widehat{\nabla}_{v_{2}} u\right) \otimes(\widehat{\Delta} u) \otimes u \otimes u\right]$

$-\frac{1}{4}(d+4)(d+6)(d+8) g^{v_{1} v_{2}} X_{7}\left[u \otimes\left(\widehat{\nabla}_{v_{1}} u\right) \otimes u \otimes\left(\widehat{\nabla}_{v_{2}} u\right) \otimes u \otimes(\widehat{\Delta} u) \otimes u\right]$

$-(d+4)(d+6)(d+8) g^{v_{1} v_{2}} X_{7}\left[u \otimes u \otimes\left(\widehat{\nabla}_{v_{1}} u\right) \otimes\left(\widehat{\nabla}_{v_{2}} u\right) \otimes(\widehat{\Delta} u) \otimes u \otimes u\right]$

$-\frac{1}{2}(d+4)(d+6)(d+8) g^{v_{1} v_{2}} X_{7}\left[u \otimes u \otimes\left(\widehat{\nabla}_{v_{1}} u\right) \otimes\left(\widehat{\nabla}_{v_{2}} u\right) \otimes u \otimes(\widehat{\Delta} u) \otimes u\right]$

$+2(d+4)(3 d+16) X_{5}\left[u \otimes u \otimes\left(\widehat{\Delta}^{v_{1} v_{2}} u\right) \otimes\left(\widehat{\nabla}_{v_{1}} u\right) \otimes\left(\widehat{\nabla}_{v_{2}} u\right)\right]$

$-4(d+4)(d+6) X_{6}\left[u \otimes u \otimes\left(\widehat{\Delta}^{v_{1} v_{2}} u\right) \otimes\left(\widehat{\nabla}_{v_{1}} u\right) \otimes u \otimes\left(\widehat{\nabla}_{v_{2}} u\right)\right]$

$-6(d+4)(d+6) X_{6}\left[u \otimes u \otimes\left(\widehat{\Delta}^{v_{1} v_{2}} u\right) \otimes u \otimes\left(\widehat{\nabla}_{v_{1}} u\right) \otimes\left(\widehat{\nabla}_{v_{2}} u\right)\right]$

$-18(d+4)(d+6) X_{6}\left[u \otimes u \otimes u \otimes\left(\widehat{\Delta}^{v_{1} v_{2}} u\right) \otimes\left(\widehat{\nabla}_{v_{1}} u\right) \otimes\left(\widehat{\nabla}_{v_{2}} u\right)\right]$

$-2(d+4)(d+6)(d+8) X_{7}\left[u \otimes u \otimes\left(\widehat{\Delta}^{v_{1} v_{2}} u\right) \otimes\left(\widehat{\nabla}_{v_{1}} u\right) \otimes\left(\widehat{\nabla}_{v_{2}} u\right) \otimes u \otimes u\right]$

$-(d+4)(d+6)(d+8) X_{7}\left[u \otimes u \otimes\left(\widehat{\Delta}^{v_{1} v_{2}} u\right) \otimes\left(\widehat{\nabla}_{v_{1}} u\right) \otimes u \otimes\left(\widehat{\nabla}_{v_{2}} u\right) \otimes u\right]$

$-2(d+2) X_{4}\left[u \otimes\left(\widehat{\nabla}_{v_{1}} u\right) \otimes\left(\widehat{\Delta}^{v_{1} v_{2}} u\right) \otimes\left(\widehat{\nabla}_{v_{2}} u\right)\right]+(d+4)(3 d+14) X_{5}\left[u \otimes\left(\widehat{\nabla}_{v_{1}} u\right) \otimes\left(\widehat{\Delta}^{v_{1} v_{2}} u\right) \otimes\left(\widehat{\nabla}_{v_{2}} u\right) \otimes u\right]$

$-4(d+4)(d+6) X_{6}\left[u \otimes\left(\widehat{\nabla}_{v_{1}} u\right) \otimes\left(\widehat{\Delta}^{v_{1} v_{2}} u\right) \otimes\left(\widehat{\nabla}_{v_{2}} u\right) \otimes u \otimes u\right]$

$-2(d+4)(d+6) X_{6}\left[u \otimes\left(\widehat{\nabla}_{v_{1}} u\right) \otimes\left(\widehat{\Delta}^{v_{1} v_{2}} u\right) \otimes u \otimes\left(\widehat{\nabla}_{v_{2}} u\right) \otimes u\right]$

$+(d+4)(d+6) X_{6}\left[u \otimes\left(\widehat{\nabla}_{v_{1}} u\right) \otimes u \otimes\left(\widehat{\Delta}^{v_{1} v_{2}} u\right) \otimes u \otimes\left(\widehat{\nabla}_{v_{2}} u\right)\right]$

$+2(d+4)(d+6) X_{6}\left[u \otimes u \otimes\left(\widehat{\nabla}_{v_{1}} u\right) \otimes\left(\widehat{\Delta}^{v_{1} v_{2}} u\right) \otimes u \otimes\left(\widehat{\nabla}_{v_{2}} u\right)\right]$

$-(d+4)(d+6)(d+8) X_{7}\left[u \otimes\left(\widehat{\nabla}_{v_{1}} u\right) \otimes u \otimes\left(\widehat{\Delta}^{v_{1} v_{2}} u\right) \otimes\left(\widehat{\nabla}_{v_{2}} u\right) \otimes u \otimes u\right]$

$-\frac{1}{2}(d+4)(d+6)(d+8) X_{7}\left[u \otimes\left(\widehat{\nabla}_{v_{1}} u\right) \otimes u \otimes\left(\widehat{\Delta}^{v_{1} v_{2}} u\right) \otimes u \otimes\left(\widehat{\nabla}_{v_{2}} u\right) \otimes u\right]$

$-2(d+4)(d+6)(d+8) X_{7}\left[u \otimes u \otimes\left(\widehat{\nabla}_{v_{1}} u\right) \otimes\left(\widehat{\Delta}^{v_{1} v_{2}} u\right) \otimes\left(\widehat{\nabla}_{v_{2}} u\right) \otimes u \otimes u\right]$

$-(d+4)(d+6)(d+8) X_{7}\left[u \otimes u \otimes\left(\widehat{\nabla}_{v_{1}} u\right) \otimes\left(\widehat{\Delta}^{v_{1} v_{2}} u\right) \otimes u \otimes\left(\widehat{\nabla}_{v_{2}} u\right) \otimes u\right]$ 
$+\frac{1}{2}(d+2)^{2} X_{4}\left[u \otimes\left(\widehat{\nabla}_{v_{1}} u\right) \otimes\left[F^{v_{1} v_{2}}, u\right] \otimes\left(\widehat{\nabla}_{v_{2}} u\right)\right]-\frac{1}{2}(d+2)(d+4) X_{5}\left[u \otimes\left(\widehat{\nabla}_{v_{1}} u\right) \otimes\left[F^{v_{1} v_{2}}, u\right] \otimes u \otimes\left(\widehat{\nabla}_{v_{2}} u\right)\right]$

$-(d+2)(d+4) X_{5}\left[u \otimes u \otimes\left(\widehat{\nabla}_{v_{1}} u\right) \otimes\left[F^{v_{1} v_{2}}, u\right] \otimes\left(\widehat{\nabla}_{v_{2}} u\right)\right]$

$-\frac{1}{4}(d+2)(d+4)(d+6) X_{6}\left[u \otimes\left(\widehat{\nabla}_{v_{1}} u\right) \otimes u \otimes\left[F^{v_{1} v_{2}}, u\right] \otimes\left(\widehat{\nabla}_{v_{2}} u\right) \otimes u\right]$

$+(d+4)(d+6) X_{4}\left[u \otimes\left(\widehat{\nabla}_{v_{1}} u\right) \otimes\left(\widehat{\nabla}_{v_{2}} u\right) \otimes\left(\widehat{\Delta}^{v_{1} v_{2}} u\right)\right]-4(d+4) X_{5}\left[u \otimes\left(\widehat{\nabla}_{v_{1}} u\right) \otimes\left(\widehat{\nabla}_{v_{2}} u\right) \otimes\left(\widehat{\Delta}^{v_{1} v_{2}} u\right) \otimes u\right]$

$-2(d+4)(d+6) X_{5}\left[u \otimes\left(\widehat{\nabla}_{v_{1}} u\right) \otimes\left(\widehat{\nabla}_{v_{2}} u\right) \otimes u \otimes\left(\widehat{\Delta}^{v_{1} v_{2}} u\right)\right]$

$+2(d+4)(d+6) X_{6}\left[u \otimes\left(\widehat{\nabla}_{v_{1}} u\right) \otimes\left(\widehat{\nabla}_{v_{2}} u\right) \otimes u \otimes u \otimes\left(\widehat{\Delta}^{v_{1} v_{2}} u\right)\right]$

$-2(d+4)(d+6) X_{6}\left[u \otimes\left(\widehat{\nabla}_{v_{1}} u\right) \otimes u \otimes u \otimes\left(\widehat{\nabla}_{v_{2}} u\right) \otimes\left(\widehat{\Delta}^{v_{1} v_{2}} u\right)\right]$

$-4(d+4)(d+6) X_{6}\left[u \otimes u \otimes\left(\widehat{\nabla}_{v_{1}} u\right) \otimes u \otimes\left(\widehat{\nabla}_{v_{2}} u\right) \otimes\left(\widehat{\Delta}^{v_{1} v_{2}} u\right)\right]$

$-6(d+4)(d+6) X_{6}\left[u \otimes u \otimes u \otimes\left(\widehat{\nabla}_{v_{1}} u\right) \otimes\left(\widehat{\nabla}_{v_{2}} u\right) \otimes\left(\widehat{\Delta}^{v_{1} v_{2}} u\right)\right]$

$-(d+4)(d+6)(d+8) X_{7}\left[u \otimes\left(\widehat{\nabla}_{v_{1}} u\right) \otimes u \otimes\left(\widehat{\nabla}_{v_{2}} u\right) \otimes\left(\widehat{\Delta}^{v_{1} v_{2}} u\right) \otimes u \otimes u\right]$

$-2(d+4)(d+6)(d+8) X_{7}\left[u \otimes u \otimes\left(\widehat{\nabla}_{v_{1}} u\right) \otimes\left(\widehat{\nabla}_{v_{2}} u\right) \otimes\left(\widehat{\Delta}^{v_{1} v_{2}} u\right) \otimes u \otimes u\right]$

$+\frac{1}{2} d(d+2) X_{4}\left[u \otimes\left(\widehat{\nabla}_{v_{1}} u\right) \otimes\left(\widehat{\nabla}_{v_{2}} u\right) \otimes\left[F^{v_{1} v_{2}}, u\right]\right]-\frac{1}{4}(d+2)(d+4)^{2} X_{5}\left[u \otimes\left(\widehat{\nabla}_{v_{1}} u\right) \otimes u \otimes\left(\widehat{\nabla}_{v_{2}} u\right) \otimes\left[F^{v_{1} v_{2}}, u\right]\right]$

$-(d+2)(d+4) X_{5}\left[u \otimes u \otimes\left(\widehat{\nabla}_{v_{1}} u\right) \otimes\left(\widehat{\nabla}_{v_{2}} u\right) \otimes\left[F^{v_{1} v_{2}}, u\right]\right]$

$+\frac{1}{2}(d+2)(d+4)(d+6) X_{6}\left[u \otimes\left(\widehat{\nabla}_{v_{1}} u\right) \otimes u \otimes u \otimes\left(\widehat{\nabla}_{v_{2}} u\right) \otimes\left[F^{v_{1} v_{2}}, u\right]\right]$

$+\frac{1}{2}(d+2)(d+4)(d+6) X_{6}\left[u \otimes u \otimes\left(\widehat{\nabla}_{v_{1}} u\right) \otimes u \otimes\left(\widehat{\nabla}_{v_{2}} u\right) \otimes\left[F^{v_{1} v_{2}}, u\right]\right]$

$-\frac{1}{2}(3 d+8) X_{3}[u \otimes(\widehat{\Delta} u) \otimes(\widehat{\Delta} u)]+(d+4) X_{4}[u \otimes(\widehat{\Delta} u) \otimes u \otimes(\widehat{\Delta} u)]+(3 d+10) X_{4}[u \otimes u \otimes(\widehat{\Delta} u) \otimes(\widehat{\Delta} u)]$

$+\frac{1}{4}(d+4)(d+6) X_{5}[u \otimes(\widehat{\Delta} u) \otimes u \otimes(\widehat{\Delta} u) \otimes u]+2(d+4) X_{5}[u \otimes(\widehat{\Delta} u) \otimes(\widehat{\Delta} u) \otimes u \otimes u]$

$+(d+4)(d+6) X_{6}[u \otimes u \otimes(\widehat{\Delta} u) \otimes(\widehat{\Delta} u) \otimes u \otimes u]$

$-4(d+4) X_{5}\left[u \otimes u \otimes\left(\widehat{\nabla}_{v_{1} v_{2}}^{2} u\right) \otimes\left(\widehat{\Delta}^{v_{1} v_{2}} u\right) \otimes u\right]+2(d+4)(d+6) X_{6}\left[u \otimes u \otimes\left(\widehat{\nabla}_{v_{1} v_{2}}^{2} u\right) \otimes\left(\widehat{\Delta}^{v_{1} v_{2}} u\right) \otimes u \otimes u\right]$

$+\frac{1}{2} d(d+2) X_{4}\left[u \otimes u \otimes\left(\widehat{\nabla}_{v_{1} v_{2}}^{2} u\right) \otimes\left[F^{v_{1} v_{2}}, u\right]\right]-(d+2)(d+4) X_{5}\left[u \otimes u \otimes u \otimes\left(\widehat{\nabla}_{v_{1} v_{2}}^{2} u\right) \otimes\left[F^{v_{1} v_{2}}, u\right]\right]$

$+2(d+2) g^{v_{1} v_{3}} g^{v_{2} v_{4}} X_{4}\left[u \otimes u \otimes\left(\widehat{\nabla}_{v_{1}} u\right) \otimes\left(\widehat{\nabla}_{v_{2} v_{3} v_{4}}^{3} u\right)\right]-2(d+4) g^{v_{1} v_{3}} g^{v_{2} v_{4}} X_{5}\left[u \otimes\left(\widehat{\nabla}_{v_{1}} u\right) \otimes u \otimes u \otimes\left(\widehat{\nabla}_{v_{2} v_{3} v_{4}}^{3} u\right)\right]$

$-2(d+4) g^{v_{1} v_{3}} g^{v_{2} v_{4}} X_{5}\left[u \otimes u \otimes u \otimes\left(\widehat{\nabla}_{v_{1}} u\right) \otimes\left(\widehat{\nabla}_{v_{2} v_{3} v_{4}}^{3} u\right)\right]$

$+(d+4)(d+6) g^{v_{1} v_{3}} g^{v_{2} v_{4}} X_{6}\left[u \otimes\left(\widehat{\nabla}_{v_{1}} u\right) \otimes u \otimes u \otimes u \otimes\left(\widehat{\nabla}_{v_{2} v_{3} v_{4}}^{3} u\right)\right]$

$-(d+4)(d+6) g^{v_{1} v_{3}} g^{v_{2} v_{4}} X_{6}\left[u \otimes u \otimes\left(\widehat{\nabla}_{v_{1}} u\right) \otimes u \otimes\left(\widehat{\nabla}_{v_{2} v_{3} v_{4}}^{3} u\right) \otimes u\right]$

$-(d+4)(d+6) g^{v_{1} v_{3}} g^{v_{2} v_{4}} X_{6}\left[u \otimes u \otimes u \otimes\left(\widehat{\nabla}_{v_{1}} u\right) \otimes\left(\widehat{\nabla}_{v_{2} v_{3} v_{4}}^{3} u\right) \otimes u\right]$

$-(d+4)(d+6) g^{v_{1} v_{3}} g^{v_{2} v_{4}} X_{6}\left[u \otimes u \otimes u \otimes\left(\widehat{\nabla}_{v_{1}} u\right) \otimes u \otimes\left(\widehat{\nabla}_{v_{2} v_{3} v_{4}}^{3} u\right)\right]$

$+2(d+4) g^{v_{1} v_{3}} g^{v_{2} v_{4}} X_{5}\left[u \otimes u \otimes u \otimes\left(\widehat{\nabla}_{v_{1} v_{2} v_{3}}^{3} u\right) \otimes\left(\widehat{\nabla}_{v_{4}} u\right)\right]$

$-(d+4)(d+6) g^{v_{1} v_{3}} g^{v_{2} v_{4}} X_{6}\left[u \otimes u \otimes u \otimes\left(\widehat{\nabla}_{v_{1} v_{2} v_{3}}^{3} u\right) \otimes\left(\widehat{\nabla}_{v_{4}} u\right) \otimes u\right]$

$+\frac{1}{6}\left(d^{2}+4 d+24\right) g^{v_{1} v_{2}} X_{3}\left[u \otimes\left(\widehat{\nabla}_{v_{1}} u\right) \otimes\left(\widehat{\nabla}_{v_{2}}(\widehat{\Delta} u)\right)\right]-\frac{1}{2}\left(d^{2}+10 d+32\right) g^{v_{1} v_{2}} X_{4}\left[u \otimes u \otimes\left(\widehat{\nabla}_{v_{1}} u\right) \otimes\left(\widehat{\nabla}_{v_{2}}(\widehat{\Delta} u)\right)\right]$

$+(d+2) g^{v_{1} v_{2}} X_{4}\left[u \otimes\left(\widehat{\nabla}_{v_{1}} u\right) \otimes u \otimes\left(\widehat{\nabla}_{v_{2}}(\widehat{\Delta} u)\right)\right]-\frac{1}{2}(d+4)(d+6) g^{v_{1} v_{2}} X_{5}\left[u \otimes\left(\widehat{\nabla}_{v_{1}} u\right) \otimes u \otimes u \otimes\left(\widehat{\nabla}_{v_{2}}(\widehat{\Delta} u)\right)\right]$

$+\frac{1}{2}(d+4)(d+14) g^{v_{1} v_{2}} X_{5}\left[u \otimes u \otimes u \otimes\left(\widehat{\nabla}_{v_{1}} u\right) \otimes\left(\widehat{\nabla}_{v_{2}}(\widehat{\Delta} u)\right)\right]-2(d+4) g^{v_{1} v_{2}} X_{5}\left[u \otimes\left(\widehat{\nabla}_{v_{1}} u\right) \otimes u \otimes\left(\widehat{\nabla}_{v_{2}}(\widehat{\Delta} u)\right) \otimes u\right]$

$-(d+4)(d+6) g^{v_{1} v_{2}} X_{6}\left[u \otimes\left(\widehat{\nabla}_{v_{1}} u\right) \otimes\left(\widehat{\nabla}_{v_{2}}(\widehat{\Delta} u)\right) \otimes u \otimes u \otimes u\right]$

$-\frac{1}{12}(d+4)(d+6) g^{v_{1} v_{2}} X_{3}\left[u \otimes\left(\widehat{\nabla}_{v_{1}} u\right) \otimes\left(\widehat{\Delta}\left(\widehat{\nabla}_{v_{2}} u\right)\right)\right]+\frac{1}{2}(d+2)(d+4) g^{v_{1} v_{2}} X_{4}\left[u \otimes u \otimes\left(\widehat{\nabla}_{v_{1}} u\right) \otimes\left(\widehat{\Delta}\left(\widehat{\nabla}_{v_{2}} u\right)\right)\right]$

$+(d+2) g^{v_{1} v_{2}} X_{4}\left[u \otimes\left(\widehat{\nabla}_{v_{1}} u\right) \otimes u \otimes\left(\widehat{\Delta}\left(\widehat{\nabla}_{v_{2}} u\right)\right)\right]+\frac{1}{2}(d+4)(d+6) g^{v_{1} v_{2}} X_{5}\left[u \otimes\left(\widehat{\nabla}_{v_{1}} u\right) \otimes\left(\widehat{\Delta}\left(\widehat{\nabla}_{v_{2}} u\right)\right) \otimes u \otimes u\right]$

$-(d+2)(d+4) g^{v_{1} v_{2}} X_{5}\left[u \otimes u \otimes u \otimes\left(\widehat{\nabla}_{v_{1}} u\right) \otimes\left(\widehat{\Delta}\left(\widehat{\nabla}_{v_{2}} u\right)\right)\right]$

$-(d+4)(d+6) g^{v_{1} v_{2}} X_{6}\left[u \otimes\left(\widehat{\nabla}_{v_{1}} u\right) \otimes\left(\widehat{\Delta}\left(\widehat{\nabla}_{v_{2}} u\right)\right) \otimes u \otimes u \otimes u\right]$

$-(3 d+10) g^{v_{1} v_{2}} X_{4}\left[u \otimes u \otimes\left(\left\{\widehat{\Delta}, \widehat{\nabla}_{v_{1}}\right\} u\right) \otimes\left(\widehat{\nabla}_{v_{2}} u\right)\right]+\frac{1}{2}(d+4)(d+6) g^{v_{1} v_{2}} X_{5}\left[u \otimes u \otimes\left(\left\{\widehat{\Delta}, \widehat{\nabla}_{v_{1}}\right\} u\right) \otimes\left(\widehat{\nabla}_{v_{2}} u\right) \otimes u\right]$ $+2(d+4) g^{v_{1} v_{2}} X_{5}\left[u \otimes u \otimes\left(\left\{\widehat{\Delta}, \widehat{\nabla}_{v_{1}}\right\} u\right) \otimes u \otimes\left(\widehat{\nabla}_{v_{2}} u\right)\right]+8(d+4) g^{v_{1} v_{2}} X_{5}\left[u \otimes u \otimes u \otimes\left(\left\{\widehat{\Delta}, \widehat{\nabla}_{v_{1}}\right\} u\right) \otimes\left(\widehat{\nabla}_{v_{2}} u\right)\right]$ 


$$
\begin{aligned}
& -(d+4)(d+6) g^{v_{1} v_{2}} X_{6}\left[u \otimes u \otimes u \otimes\left(\left\{\widehat{\Delta}, \widehat{\nabla}_{\nu_{1}}\right\} u\right) \otimes\left(\widehat{\nabla}_{v_{2}} u\right) \otimes u\right] \\
& -2(d+4) g^{v_{1} v_{3}} g^{v_{2} v_{4}} X_{5}\left[u \otimes u \otimes u \otimes u \otimes\left(\widehat{\nabla}_{v_{1} v_{2} v_{3} v_{4}}^{4} u\right)\right] \\
& +(d+2) g^{v_{1} v_{2}} X_{4}\left[u \otimes u \otimes u \otimes\left(\widehat{\nabla}_{v_{1}}\left(\widehat{\Delta}\left(\widehat{\nabla}_{v_{2}} u\right)\right)\right)\right]-2(d+4) g^{v_{1} v_{2}} X_{5}\left[u \otimes u \otimes u \otimes u \otimes\left(\widehat{\nabla}_{v_{1}}\left(\widehat{\Delta}\left(\widehat{\nabla}_{v_{2}} u\right)\right)\right)\right] \\
& -\frac{1}{2} d X_{3}[u \otimes u \otimes(\widehat{\Delta}(\widehat{\Delta} u))]+2(d+2) X_{4}[u \otimes u \otimes u \otimes(\widehat{\Delta}(\widehat{\Delta} u))]-2(d+4) X_{5}[u \otimes u \otimes u \otimes u \otimes(\widehat{\Delta}(\widehat{\Delta} u))] \\
& +\frac{1}{6} \mathfrak{R} X_{1}[q]+X_{2}[q \otimes q]+X_{3}[u \otimes(\widehat{\Delta} q) \otimes u] \\
& +\frac{1}{2}(d+2)^{2} g^{v_{1} v_{2}} X_{4}\left[u \otimes\left(\widehat{\nabla}_{v_{1}} u\right) \otimes\left(\widehat{\nabla}_{v_{2}} u\right) \otimes q\right]-\frac{1}{2}(d+2)(d+4) g^{v_{1} v_{2}} X_{5}\left[u \otimes\left(\widehat{\nabla}_{v_{1}} u\right) \otimes u \otimes\left(\widehat{\nabla}_{v_{2}} u\right) \otimes q\right] \\
& -(d+2)(d+4) g^{v_{1} v_{2}} X_{5}\left[u \otimes u \otimes\left(\widehat{\nabla}_{v_{1}} u\right) \otimes\left(\widehat{\nabla}_{v_{2}} u\right) \otimes q\right] \\
& -(d+2) g^{v_{1} v_{2}} X_{4}\left[u \otimes\left(\widehat{\nabla}_{v_{1}} u\right) \otimes q \otimes\left(\widehat{\nabla}_{v_{2}} u\right)\right]+\frac{1}{2}(d+2)(d+4) g^{v_{1} v_{2}} X_{5}\left[u \otimes\left(\widehat{\nabla}_{v_{1}} u\right) \otimes q \otimes\left(\widehat{\nabla}_{v_{2}} u\right) \otimes u\right] \\
& -(d+2) g^{v_{1} v_{2}} X_{4}\left[u \otimes q \otimes\left(\widehat{\nabla}_{v_{1}} u\right) \otimes\left(\widehat{\nabla}_{v_{2}} u\right)\right]-(d+2) g^{v_{1} v_{2}} X_{4}\left[q \otimes u \otimes\left(\widehat{\nabla}_{v_{1}} u\right) \otimes\left(\widehat{\nabla}_{v_{2}} u\right)\right] \\
& +\frac{1}{2}(d+2)(d+4) g^{v_{1} v_{2}} X_{5}\left[u \otimes q \otimes\left(\widehat{\nabla}_{v_{1}} u\right) \otimes\left(\widehat{\nabla}_{v_{2}} u\right) \otimes u\right]+\frac{1}{2}(d+2)(d+4) g^{v_{1} v_{2}} X_{5}\left[q \otimes u \otimes\left(\widehat{\nabla}_{v_{1}} u\right) \otimes\left(\widehat{\nabla}_{v_{2}} u\right) \otimes u\right] \\
& +\sum_{\ell=1}^{3}\left(i_{q}^{(\ell)} X_{3}\right)[u \otimes(\widehat{\Delta} u)]-\frac{1}{2}(d+2) \sum_{\ell=1}^{4}\left(i_{q}^{(\ell)} X_{4}\right)[u \otimes(\widehat{\Delta} u) \otimes u] \\
& +2 g^{v_{1} v_{2}} X_{3}\left[u \otimes\left(\widehat{\nabla}_{v_{1}} q\right) \otimes\left(\widehat{\nabla}_{v_{2}} u\right)\right]-(d+2) g^{v_{1} v_{2}} X_{4}\left[u \otimes\left(\widehat{\nabla}_{v_{1}} q\right) \otimes\left(\widehat{\nabla}_{v_{2}} u\right) \otimes u\right] \\
& -(d+2) g^{v_{1} v_{2}} X_{4}\left[u \otimes\left(\widehat{\nabla}_{v_{1}} u\right) \otimes\left(\widehat{\nabla}_{v_{2}} q\right) \otimes u\right] \text {. }
\end{aligned}
$$

As explained for $\mathscr{R}_{2}$, this presentation is not unique.

Remark 7.1 This series of terms may quite seem rather useless per se. But the attentive reader will see that these simplified expressions show some repeated structures, for instance the use of the inclusion operator $i_{q}^{(\ell)}$ in the form $\sum_{\ell=1}^{k} i_{q}^{(\ell)} X_{k}$ and the possible factorization by common polynomials in the dimension parameter $d$ for a large number of terms sharing the same pattern. As can be seen in Corollary 5.2, the results can also be presented in a more factorized way (something we have not really tried to get for $\mathscr{R}_{4}$ ). Splitting the results into $u$-universal operators $X_{\alpha, k}$ and $P$-dependent arguments on which they are applied was a strong motivation to get a better structural perception of heat coefficients. Hitherto, we are not in position to offer more perspectives in that regard but further investigations may reveal structures hidden so far. The computation of higher-order heat coefficients via the present method could also offer the detection of possible (hidden) structures. We encourage insightful readers to take an interest in this problem.

\subsection{If $u$ is parallel for $\nabla$}

We first remark that when $u=\mathbb{1}$, the contribution of $\mathscr{R}_{4,4}$ reduces to $(4 \pi)^{d / 2} \mathscr{R}_{4,4}=\frac{1}{12} F^{v_{1} v_{2}} F_{v_{1} v_{2}}$ (even if $N^{v}$ is non zero) while $\mathscr{R}_{4,3}$ vanishes (even if $N^{v}$ is non zero) and the contribution of $\mathscr{R}_{4,2}$ is

$$
(4 \pi)^{d / 2} \mathscr{R}_{4,2}=\left(\frac{1}{12} N^{v_{1}} N^{v_{2}}-\frac{1}{6} g^{\nu_{2} \nu_{3}}\left(\nabla_{\nu_{3}} N^{\nu_{1}}\right)\right) F_{\nu_{1} \nu_{2}} .
$$

More generally, when $u$ is parallel for the connection $\nabla$, we get the following 55 terms for $\mathscr{R}_{4}$; recall from (4.4) that $G_{v_{1} v_{2} v_{3} v_{4}}:=\frac{1}{4}\left(g_{v_{1} v_{2}} g_{v_{3} v_{4}}+g_{v_{1} v_{3}} g_{v_{2} v_{4}}+g_{v_{1} v_{4}} g_{v_{2} v_{3}}\right):$

$$
\begin{aligned}
(4 \pi)^{d / 2} \mathscr{R}_{4}= \\
\quad+\frac{1}{180}|R|^{2} X_{1}[u]-\frac{1}{180}|\operatorname{Ric}|^{2} X_{1}[u]+\frac{1}{30}(\widehat{\Delta} \Re) X_{1}[u]+\frac{1}{72} \Re^{2} X_{1}[u]+\frac{1}{6} \Re X_{1}[q]+X_{2}[q \otimes q]+X_{3}[u \otimes(\widehat{\Delta} q) \otimes u] \\
\quad+\frac{1}{12}\left(\widehat{\nabla}_{v_{1}} \Re\right) X_{1}\left[N^{v_{1}}\right]-\frac{1}{2}\left(\widehat{\nabla}_{v_{1}} \Re\right) X_{3}\left[u \otimes u \otimes N^{v_{1}}\right]+\frac{1}{6} \operatorname{Ric}_{v_{1} v_{2}} X_{2}\left[N^{v_{1}} \otimes N^{v_{2}}\right]-\frac{1}{2} \operatorname{Ric}_{v_{1} v_{2}} X_{3}\left[N^{v_{1}} \otimes u \otimes N^{v_{2}}\right] \\
\quad-\frac{1}{12} g_{v_{1} v_{2}} \Re X_{2}\left[N^{v_{1}} \otimes N^{v_{2}}\right]-\frac{1}{2} g_{v_{1} v_{2}} \sum_{\ell=1}^{3}\left(i_{q}^{(\ell)} X_{3}\right)\left[N^{v_{1}} \otimes N^{v_{2}}\right]+G_{v_{1} v_{2} v_{3} v_{4}} X_{4}\left[N^{v_{1}} \otimes N^{v_{2}} \otimes N^{v_{3}} \otimes N^{v_{4}}\right] \\
\quad-X_{3}\left[u \otimes\left(\widehat{\nabla}_{v_{1}} q\right) \otimes N^{v_{1}}\right]+X_{3}\left[N^{v_{1}} \otimes\left(\widehat{\nabla}_{v_{1}} q\right) \otimes u\right] \\
\quad+\frac{1}{3} g^{v_{1} v_{3}} \operatorname{Ric}_{v_{2} v_{3}} X_{2}\left[u \otimes\left(\widehat{\nabla}_{v_{1}} N^{v_{2}}\right)\right]-g^{v_{1} v_{3}} \operatorname{Ric}_{v_{2} v_{3}} X_{3}\left[u \otimes u \otimes\left(\widehat{\nabla}_{v_{1}} N^{v_{2}}\right)\right]-\frac{1}{6} \Re X_{2}\left[u \otimes\left(\widehat{\nabla}_{v_{1}} N^{v_{1}}\right)\right] \\
\quad-\sum_{\ell=1}^{3}\left(i_{q}^{(\ell)} X_{3}\right)\left[u \otimes\left(\widehat{\nabla}_{v_{1}} N^{v_{1}}\right)\right]
\end{aligned}
$$




$$
\begin{aligned}
& +\frac{1}{2} g_{v_{1} v_{2}} X_{3}\left[N^{v_{1}} \otimes N^{v_{2}} \otimes\left(\widehat{\nabla}_{v_{3}} N^{v_{3}}\right)\right]-\frac{1}{2} g_{v_{1} v_{2}} X_{4}\left[N^{v_{1}} \otimes N^{v_{2}} \otimes\left(\widehat{\nabla}_{v_{3}} N^{v_{3}}\right) \otimes u\right] \\
& -\frac{1}{2} g_{v_{1} v_{3}} X_{4}\left[N^{v_{1}} \otimes N^{v_{2}} \otimes\left(\widehat{\nabla}_{v_{2}} N^{v_{3}}\right) \otimes u\right]-\frac{1}{2} g_{v_{2} v_{3}} X_{4}\left[N^{v_{1}} \otimes N^{v_{2}} \otimes\left(\widehat{\nabla}_{v_{1}} N^{v_{3}}\right) \otimes u\right] \\
& +\frac{1}{2} g_{v_{1} v_{3}} X_{4}\left[N^{v_{1}} \otimes u \otimes\left(\widehat{\nabla}_{v_{2}} N^{v_{2}}\right) \otimes N^{v_{3}}\right]+\frac{1}{2} g_{v_{1} v_{3}} X_{4}\left[u \otimes N^{v_{1}} \otimes\left(\widehat{\nabla}_{v_{2}} N^{v_{2}}\right) \otimes N^{v_{3}}\right] \\
& +\frac{1}{2} g_{v_{1} v_{3}} X_{4}\left[N^{v_{1}} \otimes u \otimes\left(\widehat{\nabla}_{v_{2}} N^{v_{3}}\right) \otimes N^{v_{2}}\right]+\frac{1}{2} g_{v_{1} \nu_{3}} X_{4}\left[u \otimes N^{v_{1}} \otimes\left(\widehat{\nabla}_{v_{2}} N^{v_{3}}\right) \otimes N^{v_{2}}\right] \\
& -\frac{1}{2} g_{v_{2} v_{3}} X_{4}\left[N^{v_{1}} \otimes\left(\widehat{\nabla}_{v_{1}} N^{v_{2}}\right) \otimes N^{v_{3}} \otimes u\right]-\frac{1}{2} g_{v_{2} v_{3}} X_{4}\left[N^{v_{1}} \otimes\left(\widehat{\nabla}_{v_{1}} N^{v_{2}}\right) \otimes u \otimes N^{v_{3}}\right] \\
& +\frac{1}{2} g_{v_{2} v_{3}} X_{4}\left[u \otimes\left(\widehat{\nabla}_{v_{1}} N^{v_{1}}\right) \otimes N^{v_{2}} \otimes N^{v_{3}}\right]+\frac{1}{2} g_{v_{2} v_{3}} X_{4}\left[u \otimes\left(\widehat{\nabla}_{v_{1}} N^{v_{2}}\right) \otimes N^{v_{1}} \otimes N^{v_{3}}\right] \\
& +\frac{1}{2} g_{v_{2} v_{3}} X_{4}\left[u \otimes\left(\widehat{\nabla}_{v_{1}} N^{v_{2}}\right) \otimes N^{v_{3}} \otimes N^{v_{1}}\right] \\
& -X_{4}\left[u \otimes\left(\widehat{\nabla}_{v_{1}} N^{v_{2}}\right) \otimes\left(\widehat{\nabla}_{v_{2}} N^{v_{1}}\right) \otimes u\right]-g_{v_{2} v_{4}} g^{v_{1} v_{3}} X_{4}\left[u \otimes\left(\widehat{\nabla}_{v_{1}} N^{v_{2}}\right) \otimes\left(\widehat{\nabla}_{v_{3}} N^{v_{4}}\right) \otimes u\right] \\
& +X_{4}\left[u \otimes\left(\widehat{\nabla}_{v_{1}} N^{v_{1}}\right) \otimes u \otimes\left(\widehat{\nabla}_{v_{2}} N^{v_{2}}\right)\right]+2 X_{4}\left[u \otimes u \otimes\left(\widehat{\nabla}_{v_{1}} N^{v_{1}}\right) \otimes\left(\widehat{\nabla}_{v_{2}} N^{v_{2}}\right)\right] \\
& +\frac{1}{2} X_{3}\left[u \otimes N^{v_{1}} \otimes\left[F_{v_{1} v_{2}}, N^{v_{2}}\right]\right]-\frac{1}{2} X_{3}\left[N^{v_{1}} \otimes u \otimes\left[F_{v_{1} v_{2}}, N^{v_{2}}\right]\right] \\
& -X_{3}\left[N^{v_{1}} \otimes\left(\widehat{\Delta}_{v_{1} v_{2}} N^{v_{2}}\right) \otimes u\right]+2 X_{4}\left[N^{v_{1}} \otimes\left(\widehat{\Delta}_{v_{1} v_{2}} N^{v_{2}}\right) \otimes u \otimes u\right]+2 X_{4}\left[u \otimes u \otimes\left(\widehat{\Delta}_{v_{1} v_{2}} N^{v_{1}}\right) \otimes N^{v_{2}}\right] \\
& -\frac{1}{2} g_{v_{1} v_{2}} X_{3}\left[u \otimes\left(\widehat{\Delta} N^{v_{1}}\right) \otimes N^{v_{2}}\right]+g_{v_{1} v_{2}} X_{4}\left[u \otimes u \otimes\left(\widehat{\Delta} N^{v_{1}}\right) \otimes N^{v_{2}}\right] \\
& -\frac{1}{2} g_{v_{1} v_{2}} X_{3}\left[N^{v_{1}} \otimes\left(\widehat{\Delta} N^{v_{2}}\right) \otimes u\right]+g_{v_{1} v_{2}} X_{4}\left[N^{v_{1}} \otimes\left(\widehat{\Delta} N^{v_{2}}\right) \otimes u \otimes u\right]+2 g^{v_{1} v_{3}} X_{4}\left[u \otimes u \otimes u \otimes\left(\widehat{\nabla}_{v_{1} v_{2} v_{3}}^{3} N^{v_{2}}\right)\right] \\
& -X_{3}\left[u \otimes u \otimes\left(\left\{\widehat{\Delta}, \widehat{\nabla}_{v_{1}}\right\} N^{v_{1}}\right)\right]+2 X_{4}\left[u \otimes u \otimes u \otimes\left(\left\{\widehat{\Delta}, \widehat{\nabla}_{v_{1}}\right\} N^{v_{1}}\right)\right] \\
& +\frac{1}{2} X_{2}\left[N^{v_{1}} \otimes N^{v_{2}}\right] F_{v_{1} v_{2}}-X_{3}\left[N^{v_{1}} \otimes u \otimes N^{v_{2}}\right] F_{v_{1} v_{2}}-g^{v_{2} v_{3}} X_{3}\left[u \otimes\left(\widehat{\nabla}_{v_{3}} N^{v_{1}}\right) \otimes u\right] F_{v_{1} v_{2}} \\
& +\frac{1}{6} g^{v_{1} v_{3}} X_{1}\left[N^{v_{2}}\right]\left(\widehat{\nabla}_{v_{1}} F_{v_{2} v_{3}}\right)-g^{v_{1} v_{3}} X_{3}\left[u \otimes N^{v_{2}} \otimes u\right]\left(\widehat{\nabla}_{v_{1}} F_{v_{2} v_{3}}\right)+\frac{1}{12} X_{1}[u] F^{v_{1} v_{2}} F_{v_{1} v_{2}} \text {. }
\end{aligned}
$$

With $N^{\mu}=0$, this shrinks to 8 terms:

$$
\begin{aligned}
(4 \pi)^{d / 2} \mathscr{R}_{4}= & +\frac{1}{180}|R|^{2} X_{1}[u]-\frac{1}{180}|\operatorname{Ric}|^{2} X_{1}[u]+\frac{1}{30}(\widehat{\Delta} \mathfrak{R}) X_{1}[u]+\frac{1}{72} \mathfrak{R}^{2} X_{1}[u]+\frac{1}{12} X_{1}[u] F^{v_{1} v_{2}} F_{v_{1} v_{2}} \\
& +\frac{1}{6} \mathfrak{R} X_{1}[q]+X_{2}[q \otimes q]+X_{3}[u \otimes(\widehat{\Delta} q) \otimes u],
\end{aligned}
$$

and agrees with [12, Theorem 3.3.1] when $u=\mathbb{1}$ using (2.28).

\section{Conclusion}

In this work we have developed for a non minimal Laplace type operator a new method to compute any $\mathscr{R}_{r}$ in terms of universal operators $X_{\alpha, k}$ with explicit details of the case $r=2$. A computer code issued by this method has already produced new results for $\mathscr{R}_{4}$. This code is ready for such computations when $r \geq 6$ and moreover it can be particularized and adapted to more specific situations like the (rational) noncommutative torus, see for instance [9], or in quantum field theory as described in [13, Section 5.4]. Among possible perspectives, the method could also be generalized to operators $P$ acting on operator algebras, for instance constructed from spectral triples.

\section{Acknowledgments}

The authors are strongly indebted to Laurent Raymond for helpful discussions concerning some aspects of the computer code at the early stage of this work.

\section{References}

[1] Ivan G. Avramidi. Gauged gravity via spectral asymptotics of non-Laplace type operators. Journal of High Energy Physics, 07:030, 2004.

[2] Ivan G. Avramidi. Dirac operators in matrix geometry. International Journal of Geometric Methods in Modern Physics, 02:227-264, 2005.

[3] Ivan G. Avramidi. Non-Laplace type operators on manifolds with boundary. In Analysis, Geometry and Topology of Elliptic Operators, pages 107-140. World Scientific, 2006. 
[4] Ivan G. Avramidi. Heat kernel method and its applications. Springer, 2015.

[5] Ivan G. Avramidi and Thomas P. Branson. Heat kernel asymptotics of operators with non-Laplace principal part. Reviews in Mathematical Physics, 13(07):847-890, 2001.

[6] Ivan G. Avramidi and Thomas P. Branson. A discrete leading symbol and spectral asymptotics for natural differential operators. Journal of Functional Analysis, 190(1):292-337, 2002.

[7] Nicole Berline, Ezra Getzler, and Michele Vergne. Heat kernels and Dirac operators. Springer-Verlag, 1992.

[8] Leo Brewin. Riemann normal coordinate expansions using Cadabra. Classical and Quantum Gravity, 26:175017, 2009.

[9] Alain Connes and Farzad Fathizadeh. The term $a_{4}$ in the heat kernel expansion of noncommutative tori. arXiv:1611.09815v1 [math.QA], 2016.

[10] Peter B. Gilkey. Invariance theory, the heat equation and the Atiyah-Singer index theorem. Studies in Advanced Mathematics, CRC Press, Inc, 2 edition, 1995.

[11] Peter B. Gilkey. Geometric properties of natural operators defined by the Riemann curvature tensor. World Scientific, 2001.

[12] Peter B. Gilkey. Asymptotic formulae in spectral geometry. CRC press, 2003.

[13] Bruno Iochum and Thierry Masson. Heat trace for Laplace type operators with non-scalar symbols. Journal of Geometry and Physics, 116:90-118, 2017.

[14] Bruno Iochum and Thierry Masson. Heat asymptotics for nonminimal laplace type operators and application to noncommutative tori. Journal of Geometry and Physics, 129:1-24, 2018.

[15] Bruno Iochum and Thierry Masson. Source code available online, 2019. URL http://www . cpt.univ-mrs.fr/ masson/HEAT-TRACE/. Contact us for information and help.

[16] Takashi Sakai. On the eigen-values of Laplacian and curvature of Riemannian manifold. Tôhoku Mathematical Journal, 23:589-603, 1971.

[17] René Schilling, Renming Song, and Zoran Vontraček. Bernstein functions: Theory and Applications. De Gruyter, Berlin, 2nd edition, 2012.

[18] Dmitri Vassilevich. Heat kernel expansion: user's manual. Physics Report, 388:279-360, 2003. 